\title{
Cardiometabolic health in humans
}

Citation for published version (APA):

van den Driessche, JJ. (2019). Cardiometabolic health in humans: effects of goji berries, algae and the bacterium rhodospirillum rubrum. [Doctoral Thesis, Maastricht University]. Gildeprint en Universitaire Pers Maastricht. https://doi.org/10.26481/dis.20191217jd

Document status and date:

Published: 01/01/2019

DOI:

10.26481/dis.20191217jd

Document Version:

Publisher's PDF, also known as Version of record

\section{Please check the document version of this publication:}

- A submitted manuscript is the version of the article upon submission and before peer-review. There can be important differences between the submitted version and the official published version of record.

People interested in the research are advised to contact the author for the final version of the publication, or visit the DOI to the publisher's website.

- The final author version and the galley proof are versions of the publication after peer review.

- The final published version features the final layout of the paper including the volume, issue and page numbers.

Link to publication

\footnotetext{
General rights rights.

- You may freely distribute the URL identifying the publication in the public portal. please follow below link for the End User Agreement:

www.umlib.nl/taverne-license

Take down policy

If you believe that this document breaches copyright please contact us at:

repository@maastrichtuniversity.nl

providing details and we will investigate your claim.
}

Copyright and moral rights for the publications made accessible in the public portal are retained by the authors and/or other copyright owners and it is a condition of accessing publications that users recognise and abide by the legal requirements associated with these

- Users may download and print one copy of any publication from the public portal for the purpose of private study or research.

- You may not further distribute the material or use it for any profit-making activity or commercial gain

If the publication is distributed under the terms of Article $25 \mathrm{fa}$ of the Dutch Copyright Act, indicated by the "Taverne" license above, 


\section{Cardiometabolic health in humans: \\ effects of goji berries, algae and the bacterium Rhodospirillum rubrum}

JJ van den Driessche 
The research was performed within NUTRIM School of Nutrition and Translational Research in Metabolism and the department of Nutrition and Movement Sciences, Physiology of Human Nutrition group.

Cover design: Lucie van den Driessche, lucie.studio

Layout: José van den Driessche

Printed by: $\quad$ Gildeprint

ISBN:

978-94-6323-944-8

(c) Copyright José J. van den Driessche, Maastricht 2019. 


\title{
Cardiometabolic health in humans: \\ effects of goji berries, algae and the bacterium Rhodospirillum rubrum
}

\author{
PROEFSCHRIFT
}

Ter verkrijging van de graad van doctor aan de Universiteit Maastricht, op gezag van de Rector Magnificus, Prof. dr. Rianne M. Letschert volgens het besluit van het College van Decanen, in het openbaar te verdedigen op dinsdag 17 december 2019 om 16:00 uur

door

José Julia van den Driessche geboren te Utrecht op 27 juli 1992 


\section{Promotores}

Prof. dr. R.P. Mensink

Prof. dr. J. Plat

\section{Beoordelingscommissie}

Prof. dr. L.P.A.J. Schrauwen (voorzitter)

Prof. dr. W.H.M. Saris

Prof. dr. R.F. Witkamp (Wageningen University \& Research)

Prof. dr. E.A. Trautwein (Trautwein Consulting, Germany)

Dr. S. Baumgartner 


\section{Content}

$\begin{array}{lll}\text { Chapter } 1 & \text { General introduction } & 7\end{array}$

Chapter 2 Effects of superfoods on risk factors of metabolic syndrome: a systematic review of human intervention trials

Chapter 3 A single dose of goji berries does not affect postprandial energy expenditure and substrate oxidation in healthy, overweight men

Chapter 4 Effects of spirulina and wakame consumption on intestinal cholesterol absorption and serum lipid concentrations in non-hypercholesterolemic adult men and women

Chapter 5 Spirulina, wakame or goji berries do not lower markers of low-grade systemic inflammation in healthy subjects

Chapter 6 Effects of oven-dried Rhodospirillum rubrum intake on serum lipid concentrations and safety parameters in slightly hypercholesterolemic men: results of a first-inman randomized, placebo-controlled intervention trial

Summary

Samenvatting

Valorisation

Dankwoord

About the author

List of publications 

CHAPTER 1

General introduction 


\section{Cardiovascular disease and atherosclerosis}

Accounting for over 17 million deaths each year, cardiovascular disease (CVD) has been the major cause of mortality worldwide over the past decades. ${ }^{1}$ The most common cause of CVD is atherosclerosis, which is defined as the buildup of plaques in the arterial wall, causing narrowing of the vessel lumen and obstruction of blood flow. Lipids and inflammation are involved in the pathogenesis underlying the process of atherosclerosis. ${ }^{2}$ Major risk factors for atherosclerosis and CVD development include obesity, dyslipidemia, insulin resistance and hypertension. The presence of more than one of these metabolic disturbances increases CVD risk beyond the additive effect of the individual factors. ${ }^{3}$ Together, the cluster of these risk factors is referred to as the metabolic syndrome. Although currently not included in its definition, the presence of a pro-inflammatory state is also recognized as an important characteristic in people suffering from the metabolic syndrome.

\section{Obesity}

Overweight and obesity are the result of an energy disbalance. When energy intake exceeds energy expenditure, excess energy is stored as fat, resulting in gain of body weight. ${ }^{4}$ In order to prevent body weight gain, strategies are aimed to lower energy intake or to increase energy expenditure. A calorie-restricted diet is considered the mainstream approach to lower energy intake, but compliance is generally problematic, especially during weight maintenance after weight loss. Strategies to increase energy expenditure are therefore a good alternative.

\section{Energy expenditure \& substrate oxidation}

Total daily energy expenditure consists of three main components. ${ }^{5}$ First, resting energy expenditure (REE) refers to the energy needed to maintain basal processes of the body in resting and fasting conditions and contributes most, up to $65 \%$, to total daily energy expenditure (Figure 1.1). Second, activity-induced energy expenditure (AEE) contributes to about $25 \%$ of daily energy expenditure. The last component is the thermic effect of food. This is referred to as diet-induced thermogenesis (DIT) and is the energy needed for the intestinal absorption of nutrients, their initial metabolism, and storage when not being oxidized immediately. DIT depends on the 
composition of the diet or meal, since DIT values differ per macronutrient: $20-30 \%$ for proteins, $10-30 \%$ for alcohol, $5-10 \%$ for carbohydrates, and $0-3 \%$ for fat. When consuming a mixed regular diet, DIT represents about $10 \%$ of total daily energy expenditure. ${ }^{5}$ Lower DIT has been reported in obese versus normal weight subjects. ${ }^{5}$ Therefore, increasing energy expenditure after meal intake, without changing energy or macronutrient intake, could be beneficial to prevent the development of obesity. Also, reduced fasting fat oxidation ${ }^{6}$ and dietary fat oxidation after an oral fat load ${ }^{7}$ have been reported in relation to obesity and weight gain. Therefore, increasing fat oxidation, besides energy expenditure, is an interesting target for interventions to prevent the onset of obesity.

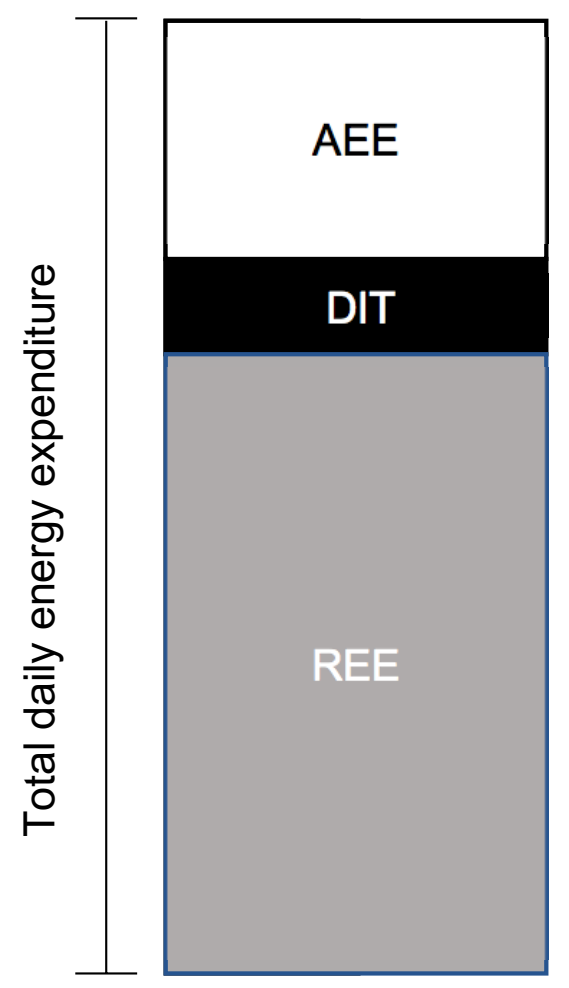

Figure 1.1: Total daily energy expenditure and its three main components: resting energy expenditure (REE), diet-induced thermogenesis (DIT) and activity-induced energy expenditure (AEE). 


\section{Measuring energy expenditure \& substrate oxidation}

Energy expenditure can be accurately estimated using indirect calorimetry. ${ }^{8}$ With this technique, oxygen consumption and carbon dioxide production are determined using a ventilated hood system. Next, total energy expenditure can be calculated. ${ }^{9}$ In addition, the ratio between oxygen consumption and carbon dioxide production, referred to as the respiratory quotient $(R Q)$, can be calculated to indicate which substrate is preferentially used by the body for oxidation at that moment in time. An $\mathrm{RQ}$ around 0.7 represents mainly fat oxidation and an $R Q$ around 1.0 mainly carbohydrate oxidation. ${ }^{10}$

\section{Dyslipidemia}

Dyslipidemia is an abnormal profile of lipids, mainly cholesterol and triacylglycerol (TAG), in the blood. Cholesterol and TAG are carried in the blood by lipoproteins (Figure 1.2). Within the exogenous pathway, cholesterol and TAG from the intestine are incorporated into chylomicrons and secreted into the circulation. By the action of lipoprotein lipase (LPL), the TAG content is released by hydrolysis and the particle shrinks to a remnant. Chylomicron remnants contain mostly esterified cholesterol and some non-hydrolyzed TAG and are taken up by the liver. The exogenous pathway becomes active in the postprandial state. ${ }^{11}$

The endogenous pathway refers to secretion of very low-density lipoprotein (VLDL) particles by the liver and the transfer of lipids to peripheral tissues. VLDL particles contain mainly TAG in its core, but also esterified cholesterol. Again, due to the action of LPL, TAG is released and delivered to peripheral tissues. Shrunken VLDL particles can be taken up by the liver or other tissues, receiving esterified cholesterol, or they can remain in the circulation and will ultimately be converted into a lowdensity lipoprotein (LDL) particle. In turn, LDL particles can be taken up by the liver or other peripheral tissues. ${ }^{11}$ 


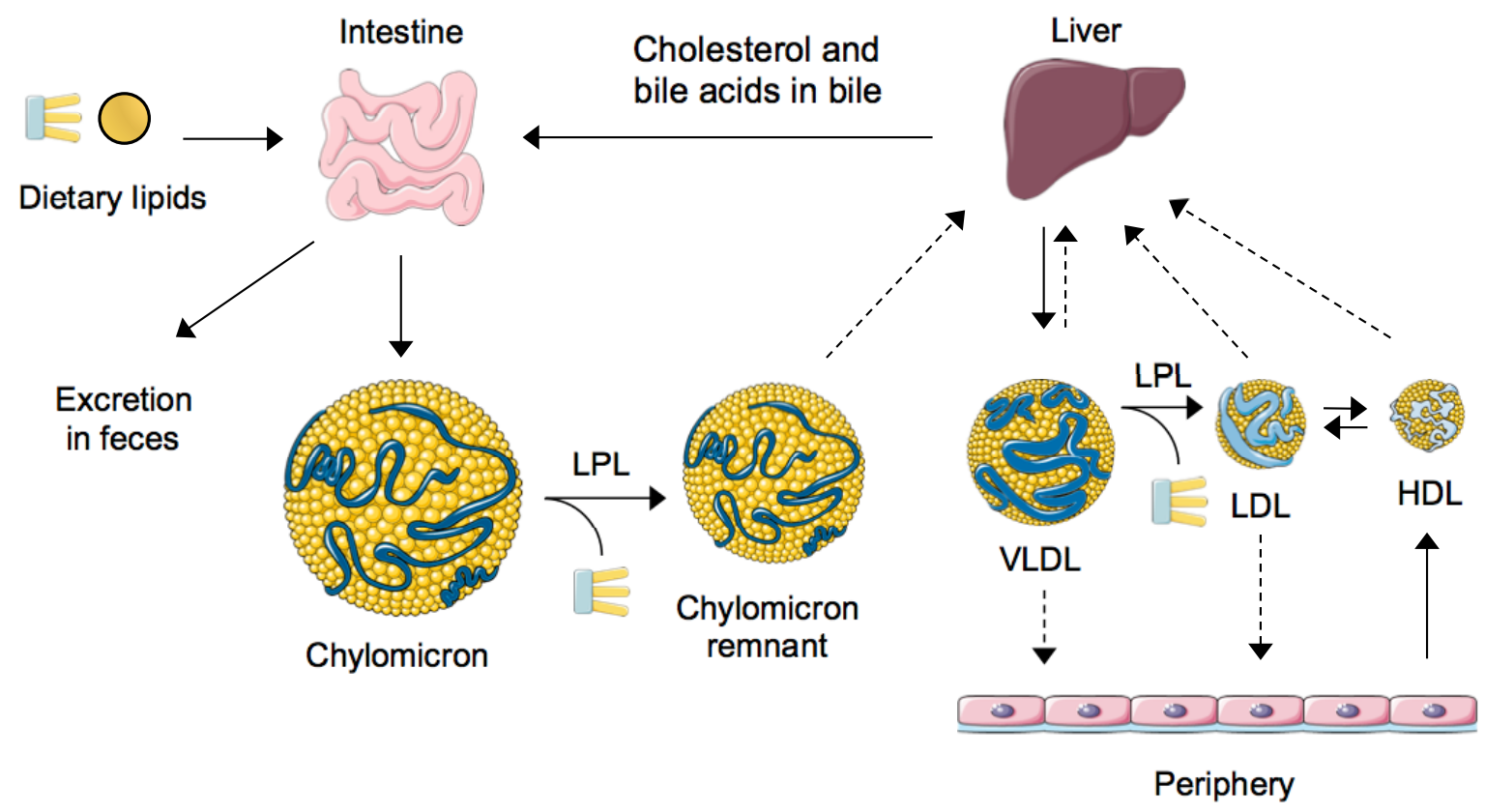

Figure 1.2: Simplified overview of lipoprotein metabolism. LPL: lipoprotein lipase; VLDL: very low-density lipoprotein; LDL: low-density lipoprotein; HDL: high-density lipoprotein. Figure was created using Servier Medical Art (https://smart.servier.com).

Last, high-density lipoprotein (HDL) is involved in the reverse cholesterol transport pathway. HDL acquires cholesterol from peripheral tissues and transports it back to the liver. In addition, HDL can also exchange cholesterol for TAG with (V)LDL. The liver can secrete cholesterol back into the intestine by excretion via bile or after conversion as bile acids. Cholesterol and bile acids are reabsorbed or excreted in the feces. ${ }^{11}$

\section{Targeting cholesterol metabolism}

Due to its role in the reverse cholesterol transport pathway, elevated HDL cholesterol is associated with a decreased CVD risk. ${ }^{11}$ On the other hand, elevated LDL cholesterol is causally related to CVD development. ${ }^{12}$ Therefore, most CVDpreventive therapies are focused on lowering LDL cholesterol concentrations. Dietary strategies to lower LDL cholesterol concentrations may be targeted at different pathways such as inhibiting endogenous cholesterol synthesis and absorption of cholesterol from the intestine. ${ }^{11}$ Nutritional interventions targeting endogenous synthesis, via inhibition of the rate-limiting enzyme HMG-CoA reductase with red yeast rice, has been shown to lower LDL cholesterol 
concentrations. ${ }^{13}$ In addition, inhibition of intestinal cholesterol absorption also lowers LDL cholesterol levels. Most well-known examples of nutritional compounds that inhibit intestinal cholesterol absorption are fibers and plant sterols and stanols. ${ }^{14,15}$ Besides lowering intestinal cholesterol absorption, fibers are also thought to inhibit reabsorption of bile acids in the intestine, stimulating synthesis of new bile acids from hepatic cholesterol, thereby stimulating the uptake of plasma LDL particles. Consequently, LDL cholesterol concentrations will decrease. ${ }^{15}$

Measuring intestinal cholesterol absorption and endogenous cholesterol synthesis Plant sterols (non-cholesterol sterols) do not only lower LDL cholesterol concentrations, but analyzing plant sterol concentrations in serum can also be used as markers for cholesterol metabolism. ${ }^{16}$ Since non-cholesterol sterols, including campesterol and sitosterol, are solely derived from the diet and are absorbed using the same transporter pathways as cholesterol, their serum concentrations can be used as markers for intestinal cholesterol absorption. However, these markers are only valid when diets are not enriched with sterols. Alternatively, the concentration of cholestanol also reflects intestinal cholesterol absorption and is also valid on a plant-sterol enriched diet. In addition, other non-cholesterol sterols, such as desmosterol and lathosterol, are precursors for the synthesis of cholesterol and their concentrations in serum can therefore be used as markers for endogenous synthesis. Since non-cholesterol sterols and cholestanol are also carried in the blood by lipoproteins, serum concentrations are usually standardized for total cholesterol concentrations.

\section{Targeting triacylglycerol metabolism}

Elevated serum levels of TAG are linked to increased CVD risk. ${ }^{11}$ High TAG levels are indicative of increased VLDL output by the liver or decreased clearance from the circulation. In postprandial conditions, chylomicrons and VLDL compete for LPL action. ${ }^{11}$ Increased fasting VLDL-TAG can therefore delay the clearance of chylomicrons after meal intake. This could result in elevated concentrations of TAGrich remnant particles, including chylomicron and VLDL remnants, which are 
believed to play an important role in atherogenesis. ${ }^{17}$ Therefore, both fasting and postprandial TAG are targets to reduce CVD risk.

\section{Insulin resistance}

When insulin resistant, the body becomes less sensitive to the actions of insulin and its physiological effects are reduced. ${ }^{11}$ Insulin is a hormone that regulates glucose metabolism in several tissues, including glucose uptake, oxidation and endogenous glucose production in the liver. However, insulin is also involved in lipid metabolism. In normal conditions, insulin inhibits VLDL production in the liver and free fatty acid (FFA) release from adipose tissue, and stimulates LPL activity, thereby promoting TAG clearance. Therefore, loss of insulin sensitivity is not only involved in the development of type II diabetes mellitus, but also relates to CVD risk. Increased fasting and/or postprandial plasma glucose concentrations may be a sign of insulin resistance. Therefore, both fasting and postprandial hyperglycemia have been linked to an increased CVD risk. ${ }^{18}$

\section{Hypertension}

High blood pressure, or hypertension, is defined as increased systolic ( $\geq 140 \mathrm{mmHg}$ ) and/or diastolic ( $\geq 90 \mathrm{mmHg}$ ) blood pressure. ${ }^{19}$ Both elevated systolic and diastolic blood pressure associate with CVD risk, but studies have indicated that an elevated systolic blood pressure is superior, especially in elderly, when assessing CVD risk. $^{19,20}$

\section{Inflammation}

For a long time, the general idea was that lipids were the main driver of atherosclerosis development. However, the importance of inflammation in the initiation and progression of atherosclerosis is being recognized more and more. ${ }^{21}$ The presence of continuous systemic inflammation is referred to as low-grade systemic inflammation and is characterized by elevated levels of circulating inflammatory markers. These markers may include acute-phase proteins, such as C-reactive protein (CRP), cytokines, including tumor necrosis factor alpha (TNFa) and interleukin 6 (IL-6), and chemokines like interleukin 8 (IL-8). Cytokines and 
chemokines can be produced by many cell types, including adipose tissue cells, endothelial cells and immune cells, mainly macrophages/monocytes. ${ }^{21}$ CRP is produced primarily in the liver in response to mainly IL-6. ${ }^{22}$

Low-grade systemic inflammation has been shown to relate to obesity and other characteristics of the metabolic syndrome. ${ }^{23}$ It has been suggested that adipose tissue dysfunction plays an important role in obesity-related low-grade systemic inflammation. ${ }^{24}$ Expansion of adipose tissue, as happens during obesity development, may ultimately lead to adipose tissue inflammation and secretion of pro-inflammatory cytokines in the circulation. Moreover, markers of low-grade systemic inflammation are independently linked to CVD risk, ${ }^{21}$ making low-grade systemic inflammation a potential target for CVD prevention. Recently, it has actually been shown that lowering inflammation, without affecting lipid profiles, lowers CVDrelated mortality. ${ }^{25}$

\section{Functional foods and superfoods}

Consumption of certain functional foods is effective in the prevention of the abovementioned CVD risk factors. ${ }^{26}$ Functional foods are characterized by their beneficial effects on physiological functions or disease risk by adding or removing certain bioactive compounds to or from foods. ${ }^{27}$ In the past years, another term to describe foods with supposedly health benefits has gained popularity. These foods, referred to as "superfoods", currently lack a clear definition and this term is mainly used as a marketing term to promote novel foods with supposedly beneficial effects on health. These claims are mostly not supported by scientific evidence of properly conducted randomized human intervention trials. However, these and other novel foods or supplements are still potential tools in CVD prevention. Therefore, we aimed to investigate the possibility to enrich the daily diet by specifically chosen foods or components to improve cardiometabolic health. 


\section{Thesis overview}

\section{Superfoods}

In chapter 2, the effects of foods labelled as superfoods on metabolic syndrome parameters are reviewed. The foods labeled as superfoods, that were reviewed, were acai berries, blueberries, cranberries, goji berries, strawberries, chili peppers, garlic, ginger, chia seed, flaxseed, hemp seed, quinoa, bee pollen, cocoa, maca, spirulina and wheatgrass. Their effects on BMI or waist circumference, systolic or diastolic blood pressure, or fasting concentrations of serum TAG, HDL-C or plasma glucose were systematically discussed.

\section{Goji berries}

Consumption of the berries from the plant Lycium barbarum, also known as goji berries or wolfberries, ${ }^{28}$ recently gained popularity within Western diets as a superfood. This classification might - among others - relate to the claimed health benefits of goji berries on energy metabolism and inflammation. Evidence for these claims is however scarce. In one human trial, goji berries increased postprandial oxygen consumption, but carbon dioxide production and consequently substrate oxidation were not reported. ${ }^{29}$ In chapter 3 of this thesis, the effects of a single dose of goji berries on postprandial energy expenditure and substrate oxidation are described. In addition, effects on postprandial TAG and glucose metabolism are presented as well, due to their link to CVD risk. The immunomodulary effects of goji berries or their extracts have been investigated in several trials, ${ }^{30-32}$ but results were not conclusive. Therefore, chapter $\mathbf{5}$ describes the effect of a single dose of goji berries on markers of low-grade systemic inflammation.

\section{Algae}

Another food that recently gained popularity within the Western diet are algae. Algae is a term including a wide variety of micro- and macro alga species, with macro algae also being referred to as seaweed. ${ }^{33}$ Spirulina (Arthrospira platensis or maxima) is one of the most-consumed and most-studied micro alga, ${ }^{34}$ while the most commonly consumed macro alga is wakame (Undaria pinnatifida). Wakame has been studied less as compared to spirulina. Several lines of evidence, including cell, animal and 
human studies, have reported LDL-cholesterol lowering effects of both algae, ${ }^{36-38}$ but results are not always in agreement ${ }^{36,39}$ and possible underlying mechanisms have not been investigated in humans yet. Therefore, the effects of spirulina and wakame consumption on intestinal cholesterol absorption, endogenous cholesterol synthesis, and serum lipid profiles were examined in this dissertation (chapter 4). Other cardiometabolic risk factors, including glucose concentrations and blood pressure, were assessed as well. In addition, some evidence suggests that these algae also exert immunomodulary effects, but studies have again not been conclusive. ${ }^{40-42}$ Chapter 5 describes the effects of spirulina and wakame consumption on markers of low-grade systemic inflammation.

\section{Rhodospirillum rubrum}

Unlike goji berries and algae, the bacterium Rhodospirillum rubrum is currently not part of any diet. Moreover, the bacterium naturally occurs in open water and moist soil. Rhodospirillum rubrum was tested in animals for its safety as a potential food source within a European Space Agency program. ${ }^{43}$ Surprisingly, cholesterollowering effects were found, attributable to the LDL fraction and not to changes in the HDL fraction. ${ }^{44}$ The hypothesis that Rhodospirillum rubrum intake lowers LDL cholesterol concentrations has however not been studied in humans so far. As part of this dissertation, the effects of oven-dried Rhodospirillum rubrum on serum lipid concentrations and safety parameters was examined for the first time in humans (chapter 6). 


\section{References}

1. World Health Organization. Cardiovascular diseases (CVD) fact sheet [Internet]. May 2017. Available from: https://www.who.int/news-room/fact-sheets/detail/cardiovascular-diseases-(cvds)

2. Weber $\mathrm{C}$, Noels $\mathrm{H}$. Atherosclerosis: current pathogenesis and therapeutic options. Nat Med. 2011;17(11):1410-22.

3. Alberti KG, Eckel RH, Grundy SM, Zimmet PZ, Cleeman JI, Donato KA, et al. Harmonizing the metabolic syndrome: a joint interim statement of the International Diabetes Federation Task Force on Epidemiology and Prevention; National Heart, Lung, and Blood Institute; American Heart Association; World Heart Federation; International Atherosclerosis Society; and International Association for the Study of Obesity. Circulation. 2009;120(16):1640-5.

4. Haslam DW, James WP. Obesity. Lancet (London, England). 2005;366(9492):1197-209.

5. Westerterp KR. Diet induced thermogenesis. Nutr Metab (Lond). 2004;1(1):5-.

6. Marra M, Scalfi L, Contaldo F, Pasanisi F. Fasting respiratory quotient as a predictor of long-term weight changes in non-obese women. Ann Nutr Metab. 2004;48(3):189-92.

7. Westerterp KR, Smeets A, Lejeune MP, Wouters-Adriaens MP, Westerterp-Plantenga MS. Dietary fat oxidation as a function of body fat. Am J Clin Nutr. 2008;87(1):132-5.

8. Ferrannini E. The theoretical bases of indirect calorimetry: a review. Metab Clin Exp. 1988;37(3):287-301.

9. Weir JB. New methods for calculating metabolic rate with special reference to protein metabolism. J Physiol. 1949;109(1-2):1-9.

10. Frayn KN. Calculation of substrate oxidation rates in vivo from gaseous exchange. J Appl Physiol: Respir Environ Exercise Physiol. 1983;55(2):628-34.

11. Frayn KN. Metabolic regulation: a human perspective: John Wiley \& Sons; 2009.

12. Ference BA, Ginsberg HN, Graham I, Ray KK, Packard CJ, Bruckert E, et al. Low-density lipoproteins cause atherosclerotic cardiovascular disease. 1. Evidence from genetic, epidemiologic, and clinical studies. A consensus statement from the European Atherosclerosis Society Consensus Panel. Eur Heart J. 2017;38(32):2459-72.

13. Gerards MC, Terlou RJ, Yu H, Koks CHW, Gerdes VEA. Traditional Chinese lipid-lowering agent red yeast rice results in significant LDL reduction but safety is uncertain - A systematic review and meta-analysis. Atherosclerosis. 2015;240(2):415-23.

14. Ras RT, Geleijnse JM, Trautwein EA. LDL-cholesterol-lowering effect of plant sterols and stanols across different dose ranges: a meta-analysis of randomised controlled studies. $\mathrm{Br} \mathrm{J}$ Nutr. 2014;112(2):214-9.

15. Gunness P, Gidley MJ. Mechanisms underlying the cholesterol-lowering properties of soluble dietary fibre polysaccharides. Food Funct. 2010;1(2):149-55.

16. Miettinen TA, Tilvis RS, Kesaniemi YA. Serum plant sterols and cholesterol precursors reflect cholesterol absorption and synthesis in volunteers of a randomly selected male population. Am J Epidemiol. 1990;131(1):20-31. 
17. Dallinga-Thie GM, Kroon J, Boren J, Chapman MJ. Triglyceride-rich lipoproteins and remnants: targets for therapy? Curr Cardiol Rep. 2016;18(7):67.

18. Ceriello A, Hanefeld M, Leiter L, Monnier L, Moses A, Owens D, et al. Postprandial glucose regulation and diabetic complications. Arch Intern Med. 2004;164(19):2090-5.

19. Kjeldsen SE. Hypertension and cardiovascular risk: General aspects. Pharmacol Res. 2018;129:95-9.

20. Vishram JK, Borglykke A, Andreasen AH, Jeppesen J, Ibsen H, Jorgensen T, et al. Impact of age on the importance of systolic and diastolic blood pressures for stroke risk: the MOnica, Risk, Genetics, Archiving, and Monograph (MORGAM) Project. Hypertension (Dallas, Tex : 1979). 2012;60(5):1117-23.

21. Ridker PM, Luscher TF. Anti-inflammatory therapies for cardiovascular disease. Eur Heart J. 2014;35(27):1782-91

22. Sproston NR, Ashworth JJ. Role of C-reactive protein at sites of inflammation and infection. Front Immunol. 2018;9:754-.

23. Emanuela F, Grazia M, Marco de R, Maria Paola L, Giorgio F, Marco B. Inflammation as a Link between obesity and metabolic Syndrome. J Nutr Metab. 2012;2012:476380.

24. van Greevenbroek MM, Schalkwijk CG, Stehouwer CD. Obesity-associated low-grade inflammation in type 2 diabetes mellitus: causes and consequences. Neth J Med. 2013;71(4):174-87.

25. Ridker PM, Everett BM, Thuren T, MacFadyen JG, Chang WH, Ballantyne C, et al. Antiinflammatory therapy with canakinumab for atherosclerotic disease. N Engl J Med. 2017;377(12):1119-31.

26. Brown L, Poudyal H, Panchal SK. Functional foods as potential therapeutic options for metabolic syndrome. Obes Rev. 2015;16(11):914-41.

27. Scientific concepts of functional foods in Europe. Consensus document. Br J Nutr. 1999;81 Suppl 1:S1-27.

28. Potterat O. Goji (Lycium barbarum and L. chinense): Phytochemistry, pharmacology and safety in the perspective of traditional uses and recent popularity. Planta Med. 2010;76(1):7-19.

29. Amagase H, Nance DM. Lycium barbarum increases caloric expenditure and decreases waist circumference in healthy overweight men and women: pilot study. J Am Coll Nutr. 2011;30(5):3049.

30. Amagase H, Sun B, Nance DM. Immunomodulatory effects of a standardized Lycium barbarum fruit juice in Chinese older healthy human subjects. J Med Food. 2009;12(5):1159-65.

31. Lee YJ, Ahn Y, Kwon O, Lee MY, Lee CH, Lee S, et al. Dietary wolfberry extract modifies oxidative stress by controlling the expression of inflammatory mRNAs in overweight and hypercholesterolemic subjects: a randomized, double-blind, placebo-controlled trial. J Agric Food Chem. 2017;65(2):309-16.

32. Vidal K, Bucheli P, Gao Q, Moulin J, Shen LS, Wang J, et al. Immunomodulatory effects of dietary supplementation with a milk-based wolfberry formulation in healthy elderly: a randomized, doubleblind, placebo-controlled trial. Rejuvenation Res. 2012;15(1):89-97.

33. Wells ML, Potin P, Craigie JS, Raven JA, Merchant SS, Helliwell KE, et al. Algae as nutritional and functional food sources: revisiting our understanding. J Appl Phycol. 2017;29(2):949-82. 
34. Buono S, Langellotti AL, Martello A, Rinna F, Fogliano V. Functional ingredients from microalgae. Food Funct. 2014;5(8):1669-85.

35. Zhang H, Pang Z, Han C. Undaria pinnatifida (Wakame): a seaweed with pharmacological properties. Sci Int. 2014;2(2):32-6.

36. Huang H, Liao D, Pu R, Cui Y. Quantifying the effects of spirulina supplementation on plasma lipid and glucose concentrations, body weight, and blood pressure. Diabetes Metab Syndr Obes. 2018;11:729-42.

37. Iritani N, Nogi J. Effect of spinach and wakame on cholesterol turnover in the rat. Atherosclerosis. 1972;15(1):87-92.

38. Yoshinaga K, Nakai Y, Izumi H, Nagaosa K, Ishijima T, Nakano T, et al. Oral administration of edible seaweed Undaria pinnatifida (Wakame) modifies glucose and lipid metabolism in rats: a DNA microarray analysis. Mol Nutr Food Res. 2018;62(12):e1700828.

39. Teas J, Irhimeh MR. Dietary algae and HIVIAIDS: proof of concept clinical data. J Appl Phycol. 2012;24(3):575-82.

40. Lee EH, Park JE, Choi YJ, Huh KB, Kim WY. A randomized study to establish the effects of spirulina in type 2 diabetes mellitus patients. Nutr Res Pract. 2008;2(4):295-300.

41. Park HJ, Lee YJ, Ryu HK, Kim MH, Chung HW, Kim WY. A randomized double-blind, placebocontrolled study to establish the effects of spirulina in elderly Koreans. Ann Nutr Metab. 2008;52(4):322-8.

42. Szulinska M, Gibas-Dorna M, Miller-Kasprzak E, Suliburska J, Miczke A, Walczak-Galezewska M, et al. Spirulina maxima improves insulin sensitivity, lipid profile, and total antioxidant status in obese patients with well-treated hypertension: a randomized double-blind placebo-controlled study. Riv Eur Sci Med Farmacol. 2017;21(10):2473-81.

43. Hendrickx L, De Wever H, Hermans V, Mastroleo F, Morin N, Wilmotte A, et al. Microbial ecology of the closed artificial ecosystem MELiSSA (Micro-Ecological Life Support System Alternative): reinventing and compartmentalizing the Earth's food and oxygen regeneration system for long-haul space exploration missions. Res Microbiol. 2006;157(1):77-86.

44. Emeis JJ, Lasseur C. United States Patent No. US 7,749,513 B2. 20 



\section{CHAPTER 2}

Effects of superfoods on risk factors of metabolic syndrome: a systematic review of human intervention trials

José J. van den Driessche, Jogchum Plat, Ronald P. Mensink 


\section{Abstract}

Background: Functional foods can be effective in the prevention of metabolic syndrome and subsequently the onset of cardiovascular diseases and type II diabetes mellitus. More recently, however, another term was introduced to describe foods with additional health benefits: "superfoods", for which up to date no generally accepted definition exists. Nonetheless, their consumption might contribute to the prevention of metabolic syndrome, for example due to the presence of potentially bioactive compounds. This review provides an overview of controlled human intervention studies with foods described as "superfoods" and their effects on metabolic syndrome parameters.

Methods: First, an Internet search was performed to identify foods described as superfoods. For these superfoods, controlled human interventions trials were identified till April 2017, investigating the effects of superfood consumption on metabolic syndrome parameters: waist circumference or BMI, blood pressure, or concentrations of HDL cholesterol, triacylglycerol or glucose.

Results \& conclusion: Seventeen superfoods were identified, including a total of 113 intervention trials: blueberries (8 studies), cranberries (8), goji berries (3), strawberries (7), chili peppers (3), garlic (21), ginger (10), chia seed (5), flaxseed (22), quinoa (1), cocoa (16), maca (1), spirulina (7), wheatgrass (1), acai berries (0), hemp seed (0) and bee pollen (0). Overall, limited evidence was found for effects of the foods described as superfoods on metabolic syndrome parameters, since results were not consistent or the number of controlled interventions trials was limited. Inconsistencies might have been related to intervention-related factors, such as duration or dose. Furthermore, conclusions may be different if other health benefits are considered. 


\section{Introduction}

Metabolic syndrome consists of a cluster of risk markers predisposing to the onset of type II diabetes mellitus and cardiovascular diseases (CVDs). ${ }^{1}$ These risk markers include dyslipidemia, hypertension, insulin resistance and abdominal obesity. Additionally, the metabolic syndrome is characterized by a pro-inflammatory and pro-thrombic state. Subjects with metabolic syndrome have a twice as high risk to develop CVDs over the next 5 to 10 years as compared with subjects without metabolic syndrome. ${ }^{1}$ For developing type II diabetes mellitus, there is even a 5 -fold higher chance.

Although different definitions exist, the diagnosis of metabolic syndrome is generally based on five factors: waist circumference or BMI, blood pressure, and fasting concentrations of plasma triacylglycerol, HDL cholesterol and glucose.$^{1}$ Improving these factors by optimizing dietary intake and composition, including the consumption of functional foods, is an effective strategy for the prevention of metabolic syndrome..$^{2-4}$

The concept of functional foods was first introduced in Japan in the 1980s. Although different definitions exist, a common characteristic is that functional foods have, as compared with regular foods, beneficial physiological effects and/or reduce the risk for disease development due to the addition or removal of certain nutrients. ${ }^{5,6}$ More recently, the term "superfoods" was introduced to describe foods with particular health benefits. The term superfood, however, is mainly used as marketing tool and no generally accepted definition exists. Many of these superfoods claim to have a wide variety of health benefits, including protection against type II diabetes mellitus and CVDs. However, these claims are frequently not strongly supported by scientific evidence, especially not by controlled human intervention trials. Still, superfood consumption could contribute to the prevention of metabolic syndrome, since many of these foods contain potentially bioactive ingredients. Therefore, the aim of the present systematic review is to provide an overview of foods described as superfoods, focusing on results of controlled human intervention studies examining parameters related to metabolic syndrome. 


\section{Methods}

\section{Selection of superfoods}

Using Google (www.google.nl), an Internet search was performed on March 3rd 2016 with the term "superfoods". The first 15 websites were screened for foods labeled as superfoods and entered into a database, which ultimately consisted of 57 foods. Each food was then categorized into one of the following food groups: vegetables; fruits; berries; fish and seafood; poultry; herbs and spices; nuts; grains, beans and legumes; seeds; dairy; and other (Table 2.1). Food groups that were part of evidence-based Dutch dietary guidelines were excluded: i.e. fruits, vegetables, fish and seafood, poultry, dairy, grains, beans and legumes, and nuts. ${ }^{7}$ Oils, green tea and red wine from the group "other" were also excluded, since these foods are part of dietary guidelines in the Netherlands. ${ }^{7}$ In the end, 17 foods labeled as superfoods were identified: acai berries, blueberries, cranberries, goji berries, strawberries, chili peppers, garlic, ginger, chia seed, flaxseed, hemp seed, quinoa, bee pollen, cocoa, maca, spirulina, and wheatgrass.

\section{Search strategy}

Potentially relevant studies were identified on April 25th 2017 through searches in Medline, Embase and Cochrane (Cochrane Central Register of Clinical Trials). For each superfood, a separate search was conducted. Search terms consisted of the superfood (Table 2.2) combined with the following keywords: "comparative study [Publication type] or randomized controlled trial [Publication type] or controlled clinical trial [Publication type]" and "cardiovascular diseases [MeSH term] or hemodynamics [MeSH term] or blood pressure [MeSH term] or hypertension [MeSH term] or lipids/blood [MeSH term] or cholesterol/blood [MeSH term] or triglycerides/blood [MeSH term] or lipoproteins/blood [MeSH term] or blood glucose [MeSH term] or insulin resistance [MeSH term] or obesity [MeSH term] or waist circumference $[\mathrm{MeSH}$ term] or weight loss [MeSH term] or body mass index [MeSH term]". 
Table 2.1: Categorization of foods identified as superfoods after an Internet search for "superfoods"

\begin{tabular}{|c|c|}
\hline Vegetables & Poultry \\
\hline Beetroot & Turkey \\
\hline Tomatoes & Herbs and spices \\
\hline Kale & Chili peppers* \\
\hline Broccoli & Garlic*$^{*}$ \\
\hline Spinach & Ginger* \\
\hline Sweet potato & Nuts \\
\hline Bok choy & Almonds \\
\hline Pumpkin & Peanuts \\
\hline Cauliflower & Pistachios \\
\hline Fruits & Walnuts \\
\hline Apples & Grains, beans and legumes \\
\hline Avocado & Black beans \\
\hline Grapefruit & Lentils \\
\hline Kiwi & Oats \\
\hline Lemons & Soybean \\
\hline Mangosteen & Seeds \\
\hline Noni fruit & Chia seed $^{*}$ \\
\hline Oranges & Flaxseed* \\
\hline Pitaya & Hemp seed* \\
\hline Pomegranates & Quinoa* $^{*}$ \\
\hline Oranges & Dairy \\
\hline Rambutan & Eggs \\
\hline Watermelon & Kefir \\
\hline Berries & Yoghurt \\
\hline Acai berries* & Other \\
\hline Blueberries* & Bee pollen* \\
\hline Cranberries* & Cocoa* $^{*}$ \\
\hline Goji berries* & Coconut oil \\
\hline Strawberries* & Green tea \\
\hline Fish and seafood & Maca* \\
\hline Mackerel & Red wine \\
\hline Salmon & Spirulina* $^{*}$ \\
\hline Sardines & Wheat grass* \\
\hline
\end{tabular}

* Foods included in this review

\section{Selection criteria}

Human intervention studies investigating the effect of one of the superfoods on waist circumference, BMI, systolic or diastolic blood pressure, triacylglycerol concentrations, HDL cholesterol concentrations or fasting glucose concentrations were selected. The selection procedure was divided in two stages: a title and abstract selection, followed by a full text selection. Papers were included if they met 
the following main criteria: 1) human intervention trial with a control group; 2) intervention with one of the selected superfoods for at least two weeks; 3) no intervention with isolated compounds or part of the superfood; 4) measurement of one or more components of the metabolic syndrome; and 5) full text available in the English language. The selection was performed by two of the researchers independently. When inconclusive, eligibility was discussed to reach consensus.

Table 2.2: Overview of the selected foods labelled as superfoods and description of search terms

\begin{tabular}{|c|c|c|}
\hline Food & Food group & Database search term \\
\hline Acai berries & Berries & ("acai") or "Euterpe oleracea" \\
\hline Blueberries & Berries & (blueberr*) or "Vaccinium corymbosum" \\
\hline Cranberries & Berries & (cranberr") or "Vaccinium macrocarpon" \\
\hline Goji berries & Berries & ((“goji”) or "Lycium Barbarum”) or "wolfberry" \\
\hline Strawberries & Berries & (strawberr*) or "Fragaria" \\
\hline Chili pepper & Herbs and spices & ((“chili pepper”) or "Capsicum”) or "chili” \\
\hline Garlic & Herbs and spices & ("garlic") or "Allium sativum" \\
\hline Ginger & Herbs and spices & ("ginger") or "Zingiber officinale" \\
\hline Chia seeds & Seeds & ("chia seed") or "Salvia hispanica" \\
\hline Flaxseed & Seeds & ("flaxseed") or "Linum usitatissimum" \\
\hline Hemp seed & Seeds & "hemp seed" \\
\hline Quinoa & Seeds & ("quinoa") or "Chenopodium quinoa" \\
\hline Bee pollen & Other & "bee pollen" \\
\hline Cocoa & Other & "cocoa" \\
\hline Maca & Other & (“maca") or "Lepidium meyenii” \\
\hline Spirulina & Other & ("spirulina") or “Arthrospira" \\
\hline Wheatgrass & Other & ("wheat grass") or "wheatgrass" \\
\hline
\end{tabular}

These foods were selected from the database as presented in table 1.

\section{Data collection}

From each of the selected papers, information regarding the design (parallel or cross-over), product, amount, duration, and population was extracted and entered into a database. In addition, data on BMI, waist circumference, systolic and diastolic blood pressure, and fasting concentrations of triacylglycerol, HDL cholesterol and glucose were collected. If necessary, units were converted into centimeters for waist 
circumference and into $\mathrm{mmol} / \mathrm{L}$ for triacylglycerol, $\mathrm{HDL}$ cholesterol and glucose concentrations. For studies with parallel designs, effects of the intervention were defined as the difference between the change from baseline after superfood consumption and the change from baseline after control product consumption. The intervention effect in crossover studies was defined as the difference between postintervention values after superfood and control product consumption.

\section{Results}

\section{Search results}

A total of 988 papers were retrieved from the selected databases. Titles and abstracts were screened and 864 papers were excluded based on the predefined selection criteria. The full texts of 124 papers were reviewed and 18 papers were excluded for not meeting the inclusion criteria. Twelve additional papers were included after a search through literature lists of the included papers. In the end, 113 intervention trials met the inclusion criteria, which were published in 118 different articles (Figure 2.1).

\section{Blueberries}

Blueberries (Vaccinium corymbosum L.) were first cultivated in the United States, but are currently grown all over the world. The berries are rich in flavonoids, mainly anthocyanidins. $^{8}$

Eight studies were identified (Table 2.3). Effects on systolic and diastolic blood pressure were investigated in different population groups: healthy subjects, ${ }^{9,10}$ subjects with the metabolic syndrome, ${ }^{11,12}$ (pre-)hypertensive women, ${ }^{13}$ smokers, ${ }^{14}$ and obese and insulin resistant subjects. ${ }^{15}$ In two studies, systolic and diastolic blood pressure decreased, ${ }^{11,13}$ while in one study only a decrease in systolic blood pressure was found. ${ }^{9}$ Nyberg et al ${ }^{16}$ observed an increase in glucose concentrations, but Basu et al did not. ${ }^{11}$ No effects were found on BMI, ${ }^{10,12-15}$, waist circumference, ${ }^{11,13}$ or triacylglycerol and HDL cholesterol concentrations. ${ }^{10-12,13,16}$ 
Figure 2.1: Flow diagram of the selection process

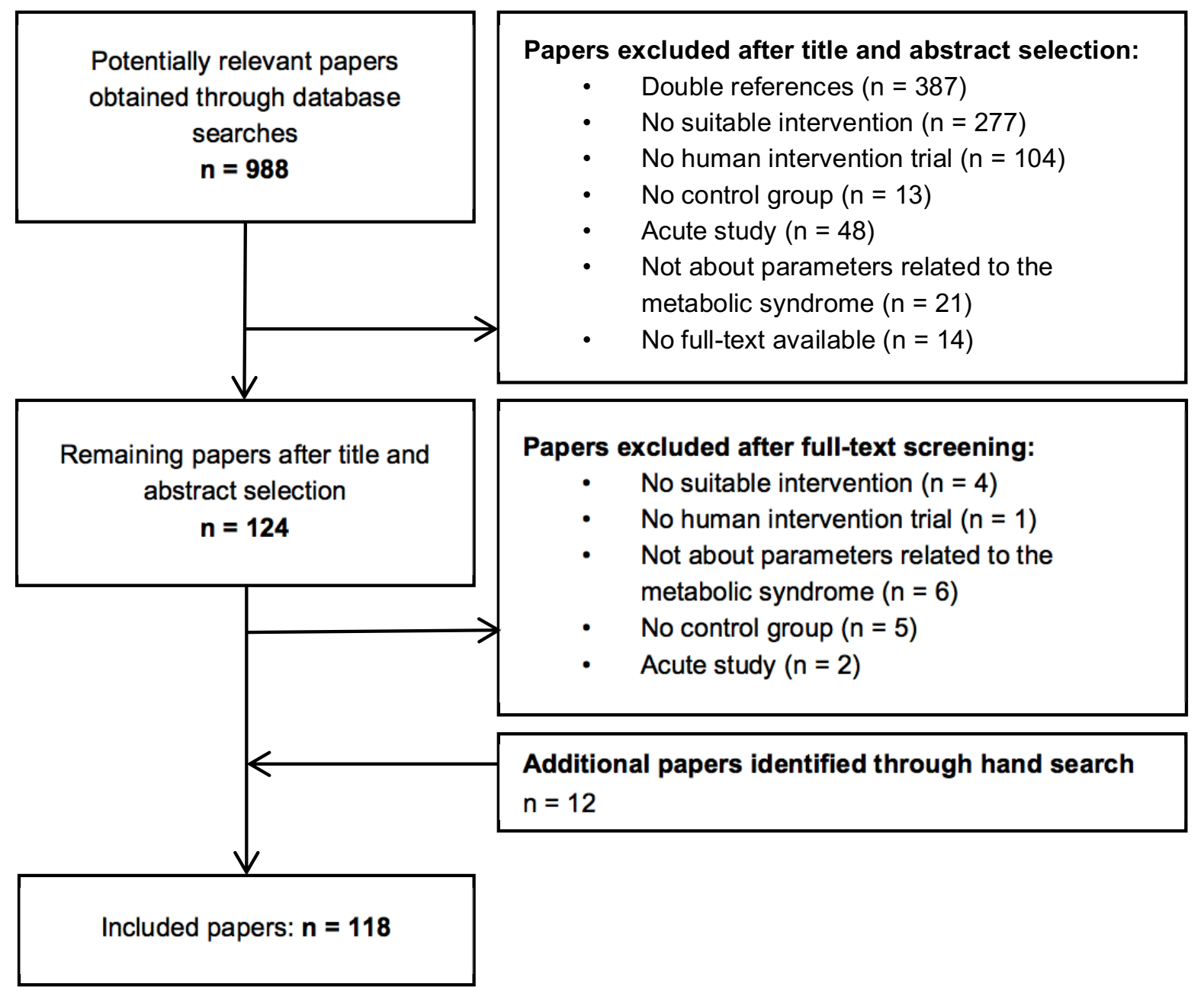




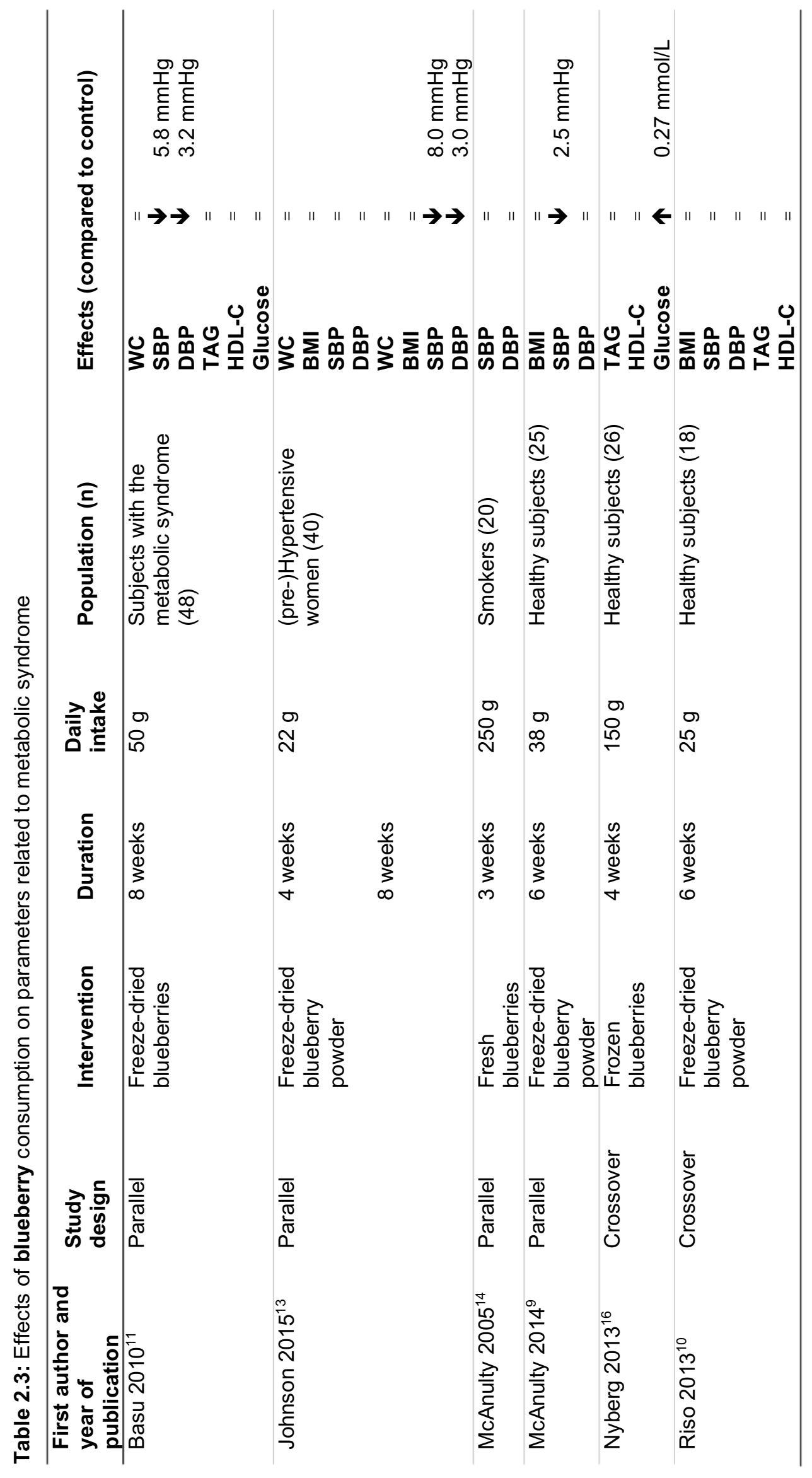




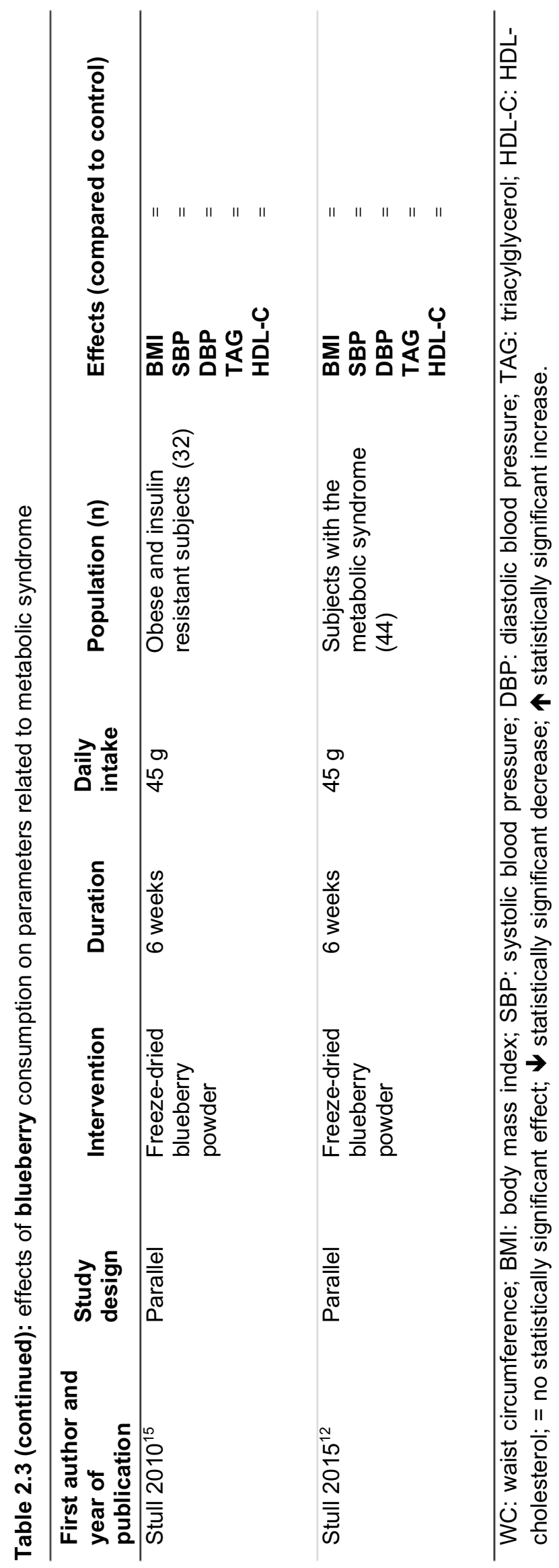




\section{Cranberries}

Cranberries, which are rich in pro-anthocyanidins, ${ }^{17}$ are cultivated all over the world including North America (Vaccinium macrocarpon Aiton) and Europe (Vaccinium oxycoccus L.). ${ }^{18}$

Eight studies matched the inclusion criteria (Table 2.4). In one study with healthy subjects, diastolic blood pressure, triacylglycerol concentrations and glucose concentrations decreased after cranberry juice consumption. ${ }^{19}$ However, these results were not supported by another trial with healthy subjects ${ }^{20}$ or by studies with subjects with metabolic syndrome, ${ }^{21,22}$ type II diabetics ${ }^{23,24}$ or subjects with (increased risk of) CVDs. ${ }^{25,26}$ In the study of Dohadwala et $\mathrm{al}^{25}$ in stable CAD patients, HDL cholesterol concentrations decreased. However, no effects on HDL cholesterol were reported in other population groups. ${ }^{20-24,26}$ None of the studies that measured waist circumference, $\mathrm{BMI}^{24}$ or systolic blood pressure ${ }^{19,21,22,24-26}$ found significant changes.

\section{Goji berries}

Goji berries (Lycium barbarum L.) have been used in traditional Asian medicine for centuries, particularly in China. Lycium barbarum polysaccharides (LBP) are thought to be the main active component in goji berries. ${ }^{27}$

Three studies met the inclusion criteria (Table 2.5), which were all carried out in healthy subjects with goji berry drinks standardized for LBP. In one study, waist circumference decreased. ${ }^{28}$ However, BMI was not altered in another study. ${ }^{29}$ No effects were found on systolic and diastolic blood pressure, and triacylglycerol and glucose concentrations. ${ }^{29,30}$ 


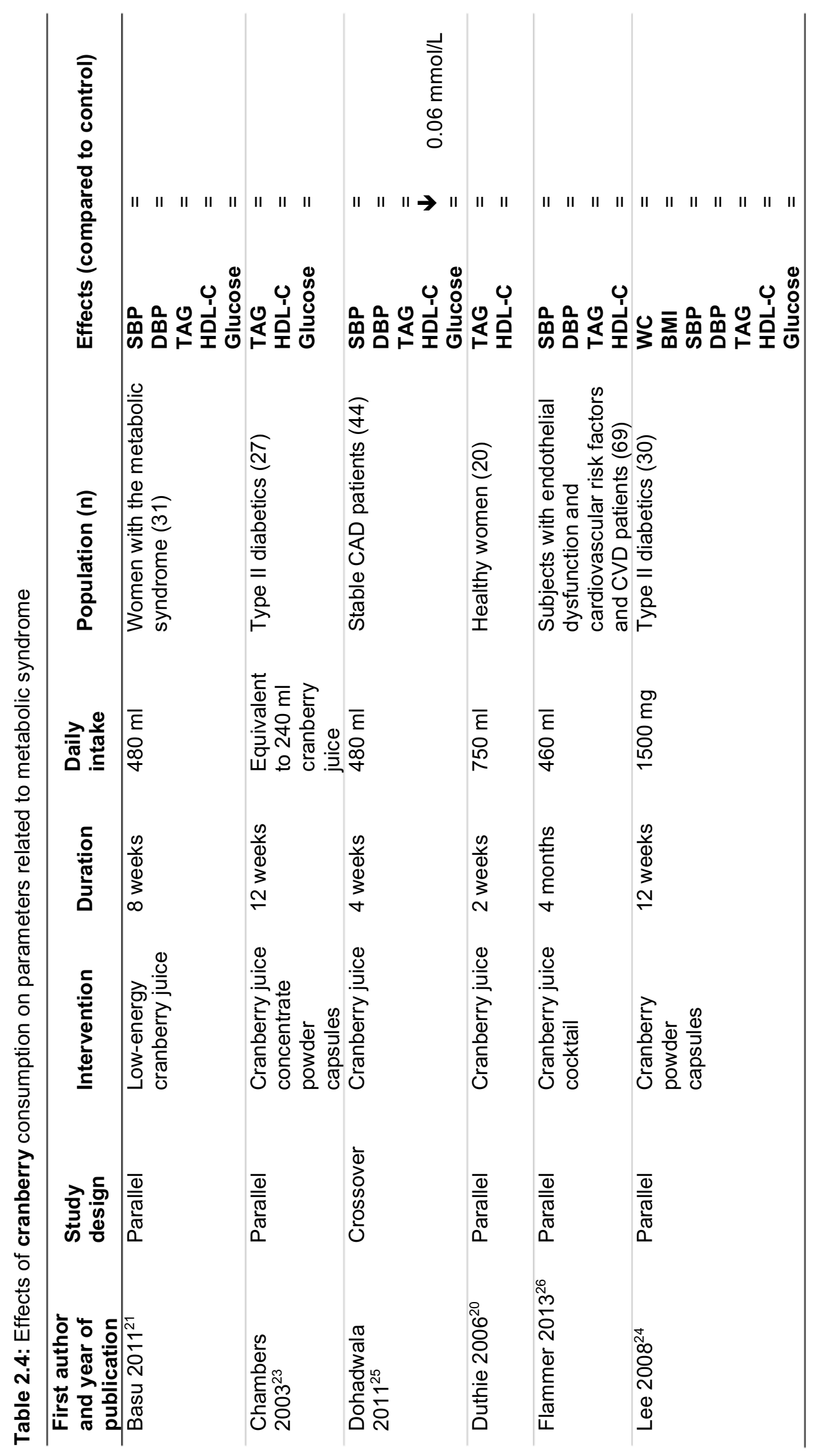




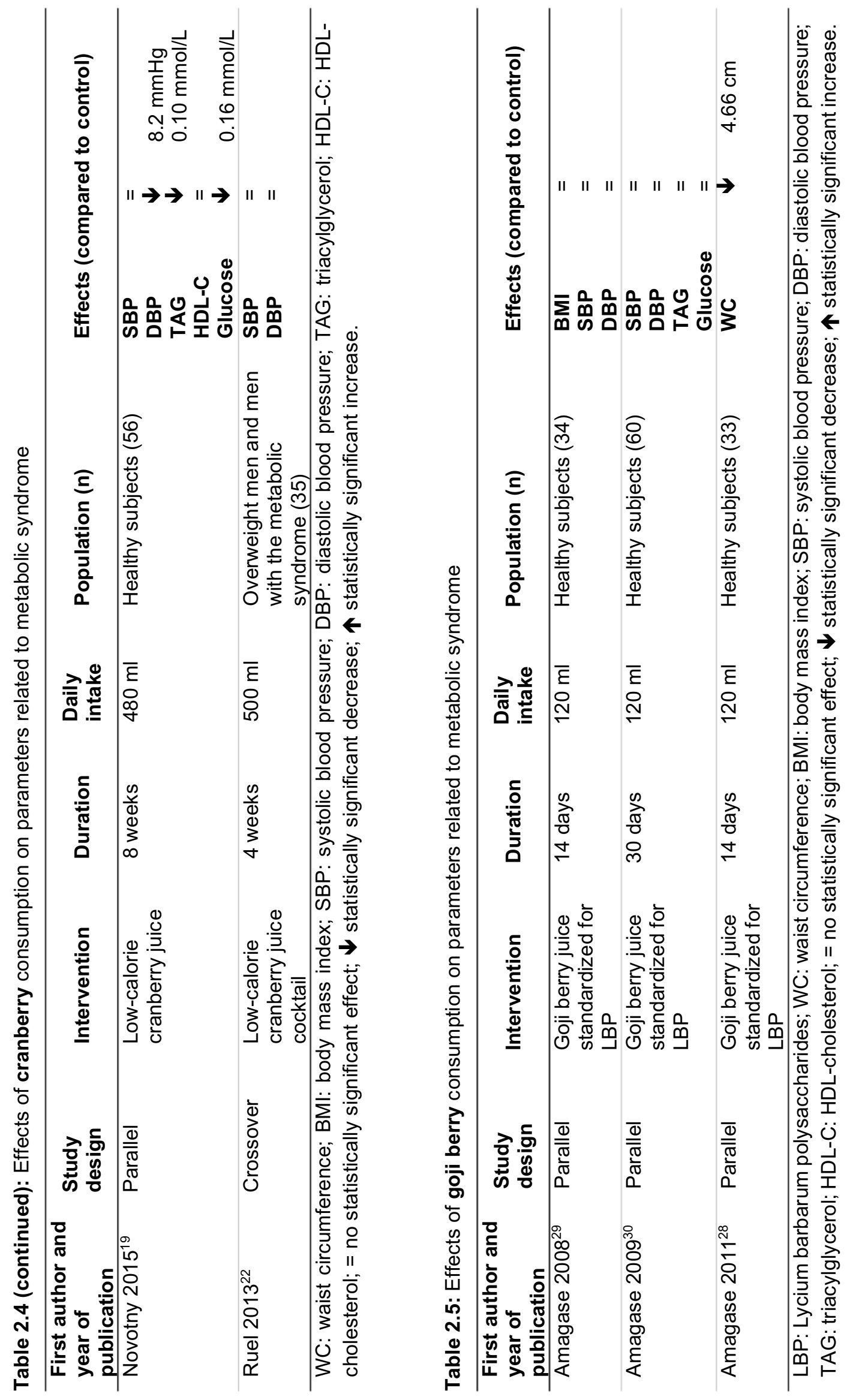




\section{Strawberries}

Strawberries (Fragaria $x$ ananassa Duchesne) were the last superfood identified in the category berries. They are cultivated and consumed worldwide, and are rich in vitamin C. ${ }^{31}$

Seven studies were identified (Table 2.6). None of the studies found effects on waist circumference or $\mathrm{BMI},{ }^{32,33}$ systolic or diastolic blood pressure, ${ }^{32-34} \mathrm{HDL}$ cholesterol concentrations, ${ }^{32-36}$ triacylglycerol concentrations, ${ }^{32-36}$ or glucose concentrations. $^{32,33,36-38}$

\section{Chili pepper}

Peppers from the plant Capsicum annuum L. are known for their pungent activity and used to spice dishes. The component of chili peppers responsible for this pungency is capsaicin. ${ }^{39}$

Three studies were identified (Table 2.7). No effects on parameters related to metabolic syndrome were reported. ${ }^{40-43}$

\section{Garlic}

Garlic (Allium sativum L.) is formally a vegetable, but essentially used to flavor dishes. Its characteristic flavor is mainly caused by sulfur compounds, which are thought to be the main active compounds in garlic. ${ }^{44}$

Twenty-one studies were identified (Table 2.8). In hypertensive subjects, systolic and diastolic blood pressure were reduced after garlic powder consumption. ${ }^{45} \mathrm{In}$ patients with peripheral artery disease ${ }^{46}$ and subjects at increased risk for ischemic attack, ${ }^{47}$ only diastolic blood pressure was reduced. However, no changes in blood pressure were found in trials with hypercholesterolemic subjects, ${ }^{48-51}$ pregnant women at risk for pre-eclampsia, ${ }^{52,53} \mathrm{CAD}$ patients, ${ }^{54}$ and healthy men and women. ${ }^{55}$ Triacylglycerol concentrations were lowered in one study with hypercholesterolemic subjects ${ }^{56}$ and one study with type II diabetics, ${ }^{57}$ but results were not confirmed in eight other studies with hypercholesterolemic subjects, ${ }^{48-51,58-63}$ type II diabetics, ${ }^{64}$ pregnant women at risk for pre-eclampsia, ${ }^{52,53} \mathrm{CAD}$ patients, ${ }^{54}$ healthy men and women, ${ }^{55}$ or overweight smokers. ${ }^{65}$ In the same studies, HDL cholesterol concentrations were increased in three studies, ${ }^{57,61,64}$ but remained unchanged in 
the other studies. Glucose concentrations were decreased after garlic consumption in type II diabetics, ${ }^{57}$ but not in pregnant women at risk for pre-eclampsia ${ }^{52}$ or hypercholesterolemic subjects. ${ }^{49} \mathrm{BMI}$, which was measured in one study, was not altered after garlic consumption. ${ }^{52}$

\section{Ginger}

The last superfood identified in the category herbs and spices is ginger. The rhizome or root of the plant Zingiber officinale Roscoe, known as ginger, is commonly used to spice dishes due to its characteristic taste. This taste is caused by gingerols and shogarols, but ginger also contains other potentially bioactive compounds such as terpenes and oleoresin. ${ }^{66,67}$

Ten studies were found (Table 2.9). Triacylglycerol concentrations were measured in five studies. In studies with hypercholesterolemic subjects, ${ }^{68}$ type II diabetics ${ }^{69,70}$ and patients on ambulatory dialysis, ${ }^{71,72}$ triacylglycerol concentrations were lowered. In CAD patients, triacylglycerol concentrations remained unchanged. ${ }^{73} \mathrm{HDL}$ cholesterol concentrations were measured in the same five studies, but no changes were found. In three studies with type II diabetics, ${ }^{69,74,77}$ a study with patients on continuous ambulatory dialysis ${ }^{71,72}$ and a study with obese women, ${ }^{76}$ glucose concentrations were lowered. In another study with type II diabetics and a study with CAD patients, however, glucose concentrations did not change. ${ }^{70,73}$ In one study with obese women, ${ }^{75}$ ginger powder decreased waist circumference and BMI, which remained unchanged in trials with type II diabetics ${ }^{69,70,74,77,78}$ and patients on ambulatory dialysis. ${ }^{71,72}$ Systolic blood pressure, but not diastolic blood pressure, was decreased in type II diabetics. ${ }^{78}$ 


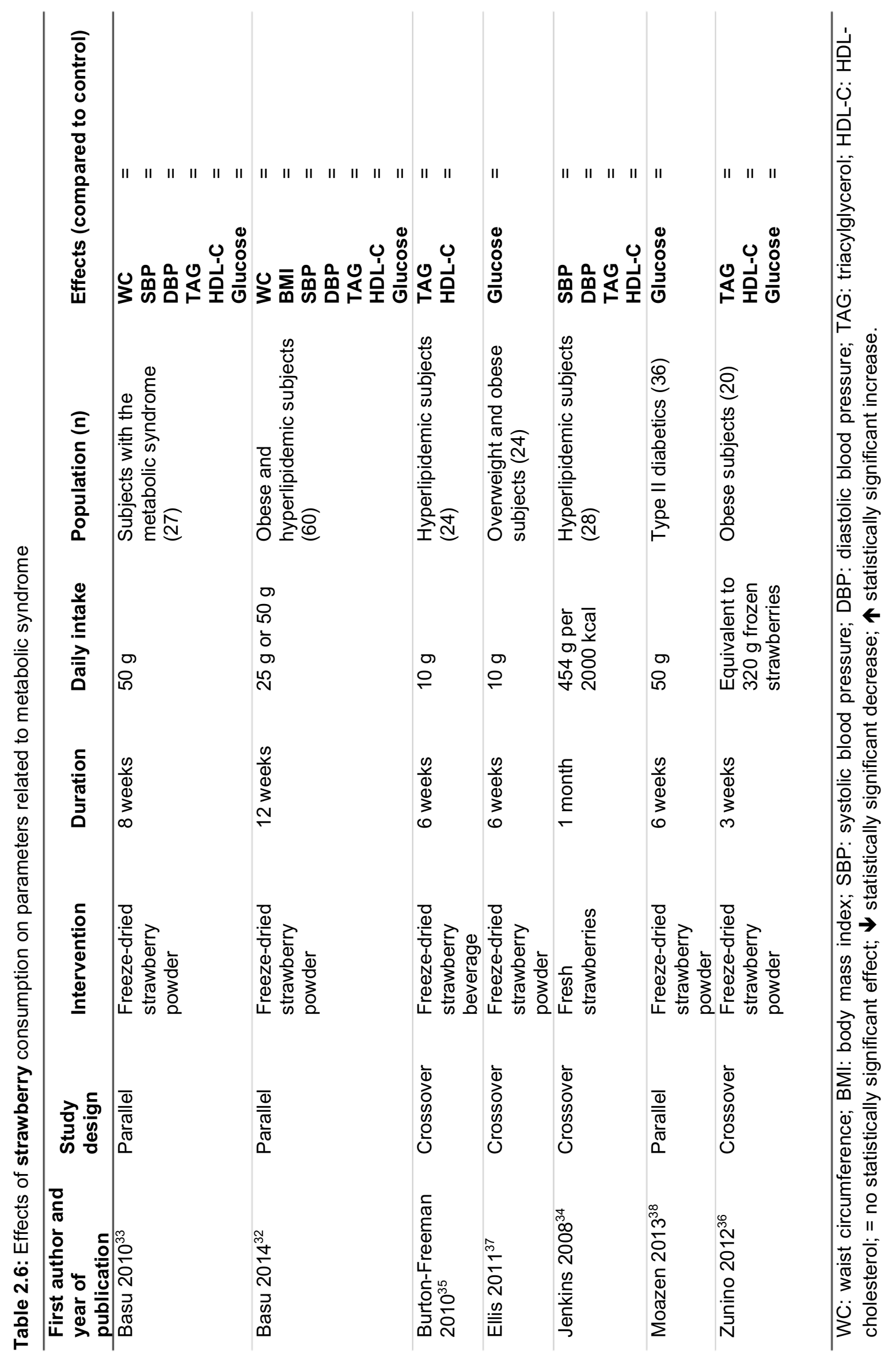



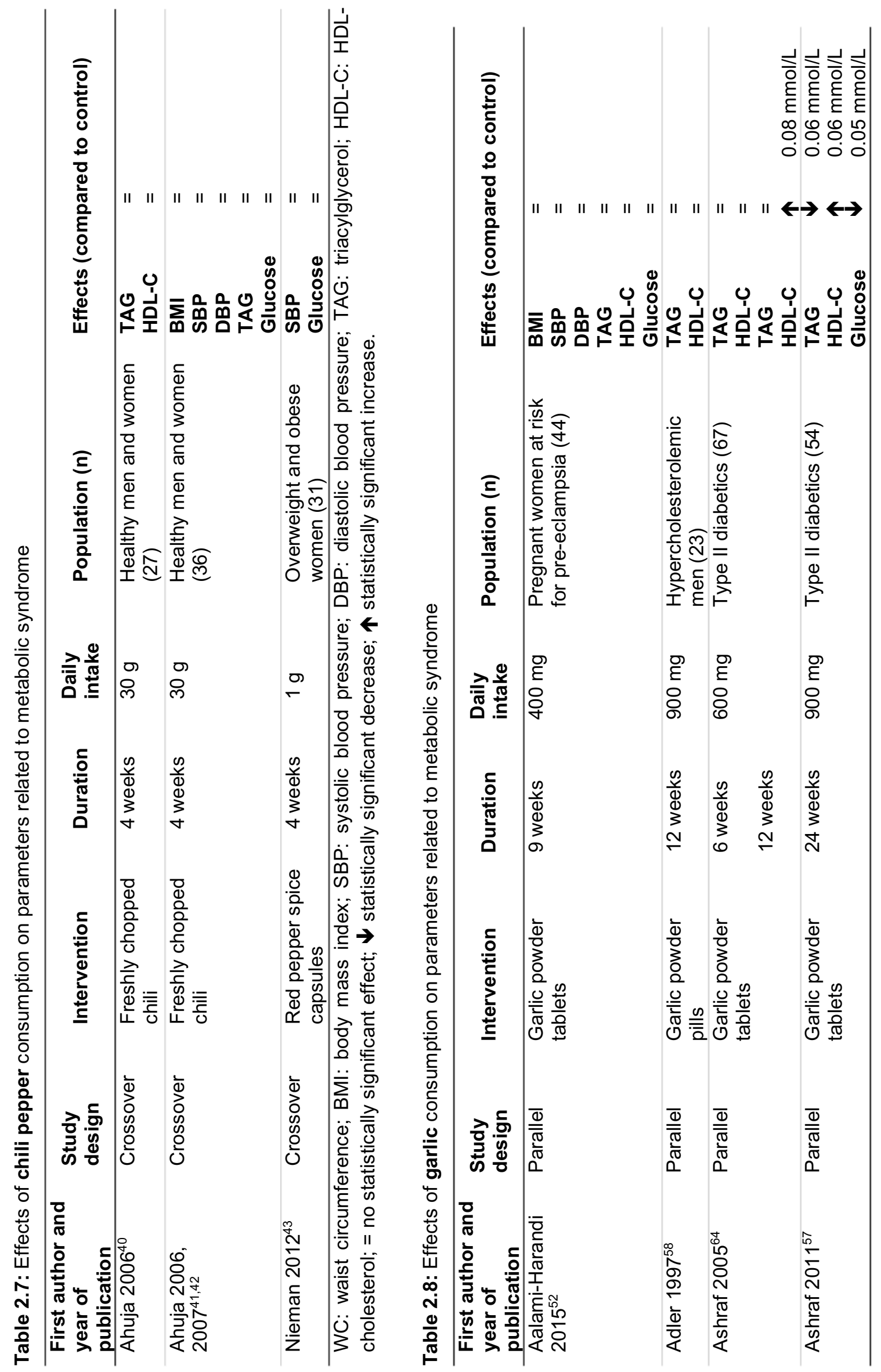


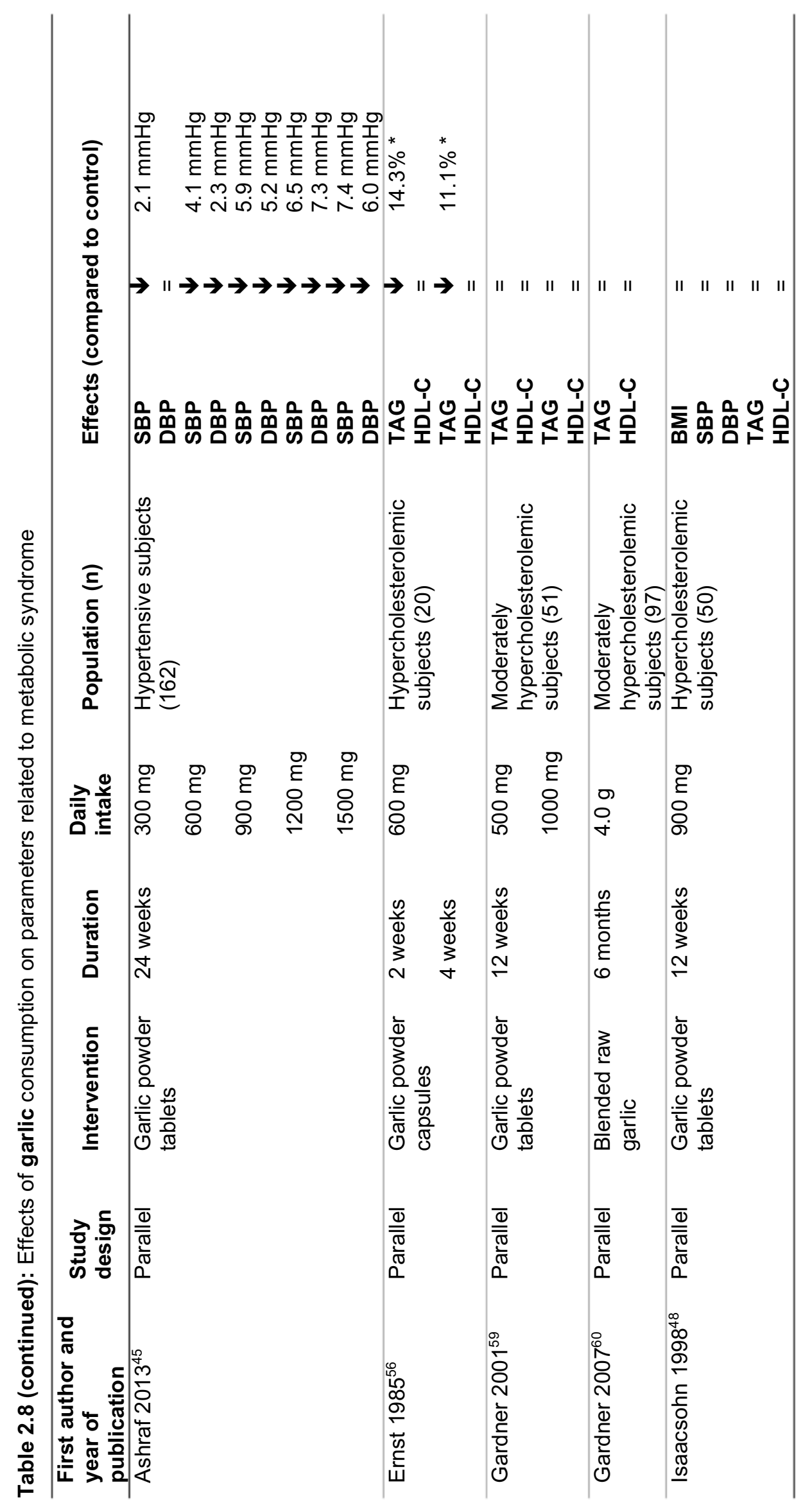




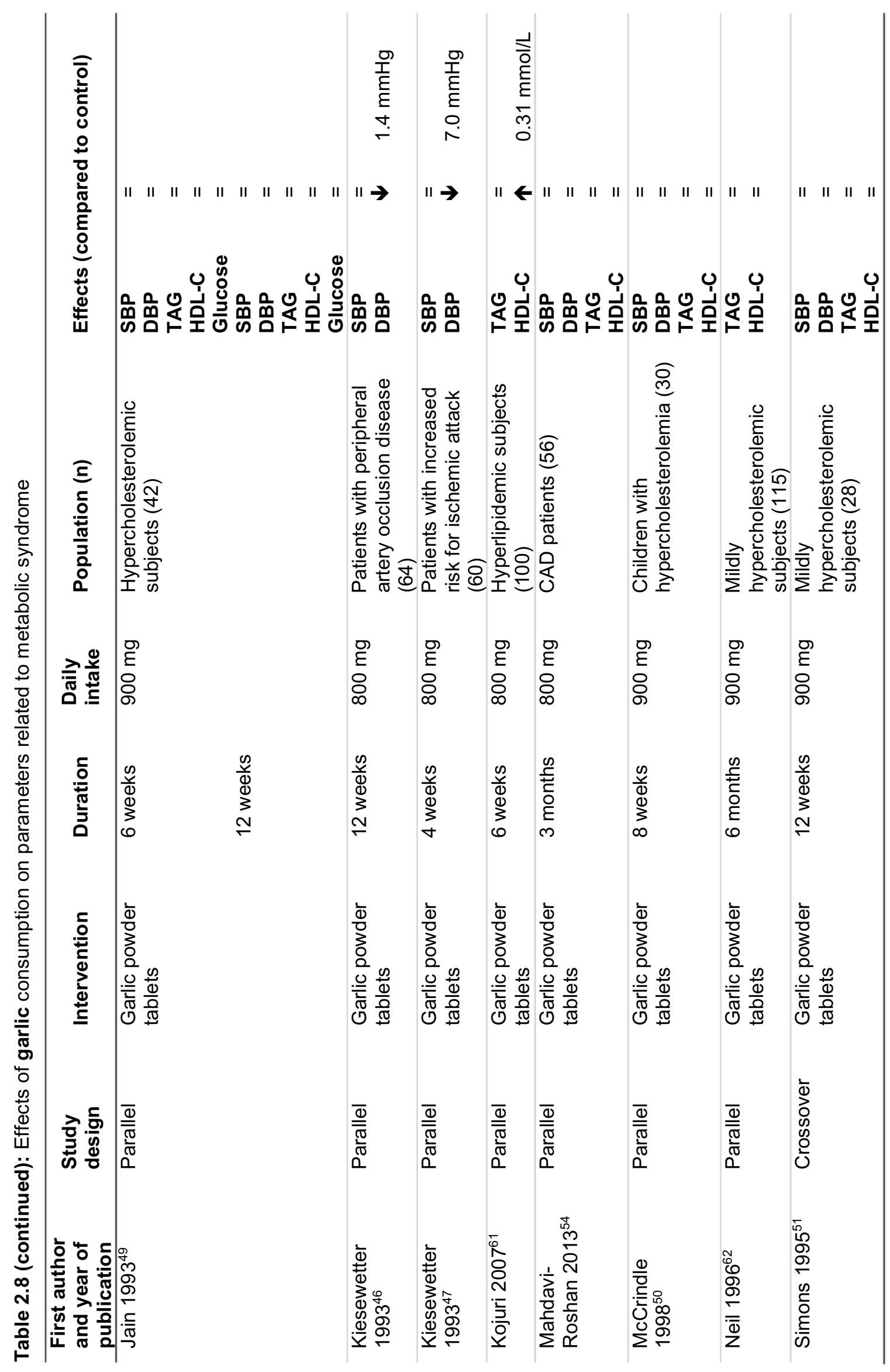



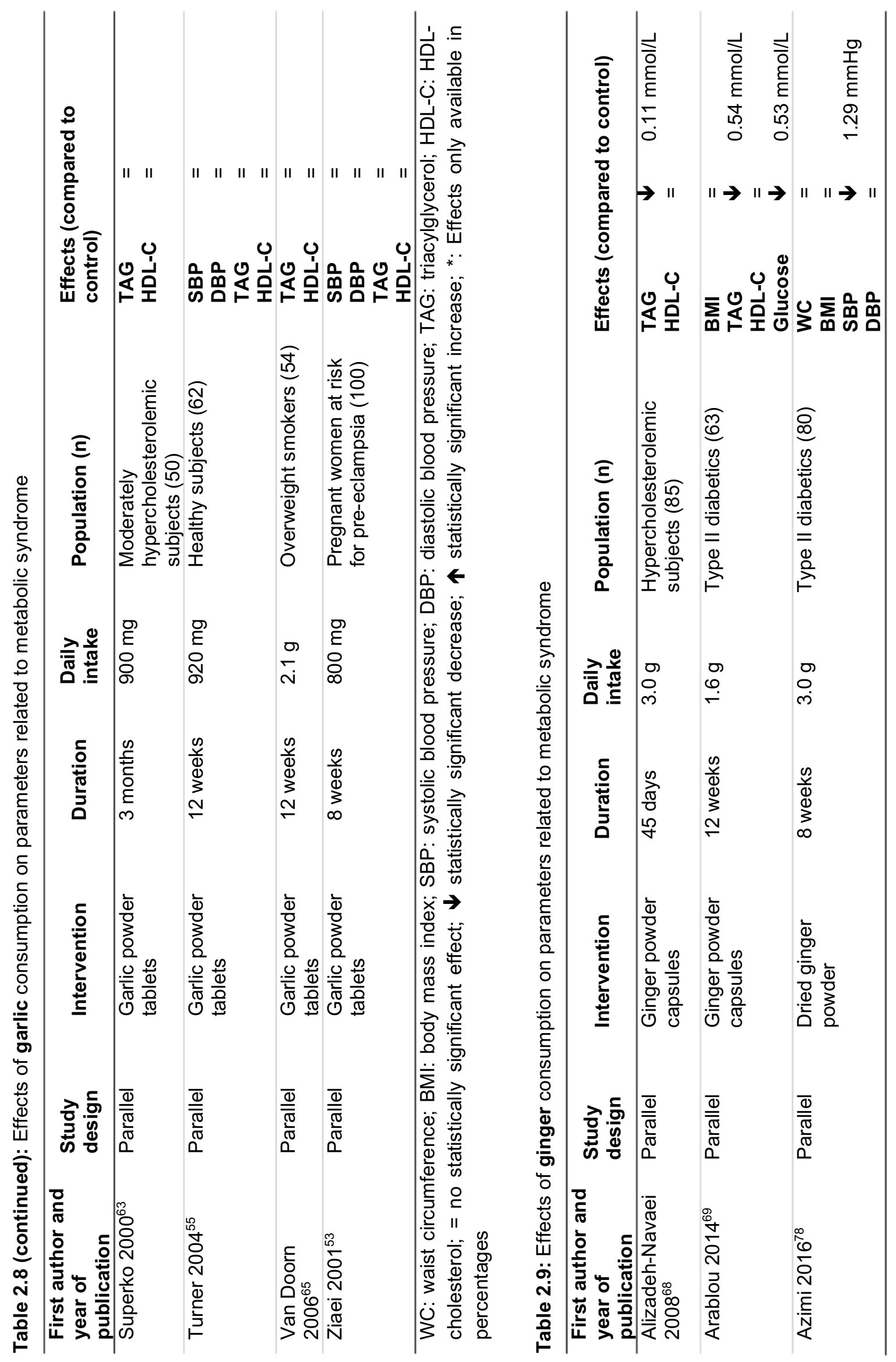


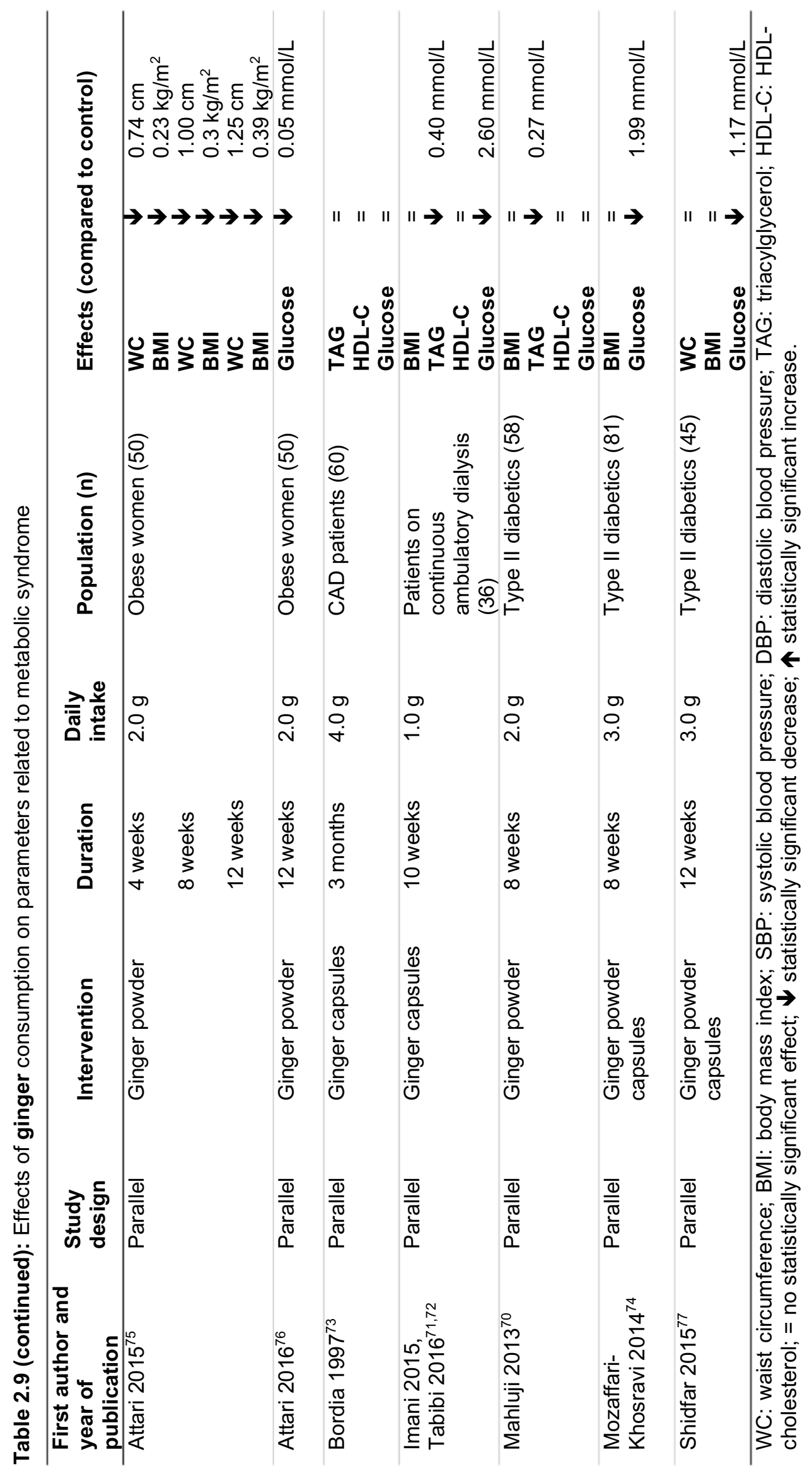




\section{Chia seeds}

Chia seeds, harvested from Salvia hispanica L., are rich in alpha-linoleic acid (ALA), which is about $75 \%$ of the oil content, and in soluble and insoluble fibers. ${ }^{79}$

Five studies were found (Table 2.10). Systolic and diastolic blood pressure decreased in a study with hypertensive subjects, ${ }^{80}$ whereas only systolic blood pressure decreased in a study with type II diabetics. ${ }^{81}$ However, in two trials with overweight and obese subjects, no effects on systolic blood pressure were found. ${ }^{82,83}$ None of the studies found effects on waist circumference or BMI, ${ }^{84}$ or concentrations of triacylglycerol or HDL cholesterol ${ }^{81,82,84}$ and glucose..$^{81-84}$

\section{Flaxseed}

The seeds of the flowering herb flax (Linum usitassimum L.) have been consumed since ancient times. ${ }^{85}$ Flaxseed is known for its high proportion of ALA, which is about $55 \%$ of all fatty acids, and for its high lignan content. 86,87

Twenty-two studies were identified (Table 2.11). Twelve studies measured waist circumference, BMI or both. One study found a decrease in both waist circumference and $\mathrm{BMI},{ }^{88}$ five studies in $\mathrm{BMI},{ }^{89-93}$ and one study found a decrease in waist circumference. ${ }^{94}$ In contrast, no changes in BMI were observed in six other studies. ${ }^{95-100}$ Systolic and diastolic blood pressure decreased in PAD patients ${ }^{100}$ and dyslipidemic subjects ${ }^{91}$. In contrast, systolic and diastolic blood pressure did not change in healthy subjects, ${ }^{89}$ overweight adolescents ${ }^{97}$ and subjects with metabolic syndrome. ${ }^{94,101}$ Results on triacylglycerol concentrations are also conflicting. Triacylglycerol concentrations were lowered in seven studies, ${ }^{90-94,102,103}$ but remained unchanged in the other ten studies. ${ }^{89,95-98,101,104-107}$ In one study, HDL cholesterol concentrations decreased, ${ }^{89}$ while in three other studies HDL cholesterol concentrations increased, ${ }^{90,91,102}$ and did not change in thirteen other studies. ${ }^{92-}$ 98,101,103-107 Finally, glucose concentrations were lowered in two studies, ${ }^{99,108}$ but not in the other five studies measuring glucose concentrations. ${ }^{94,97,106,109,110}$ 


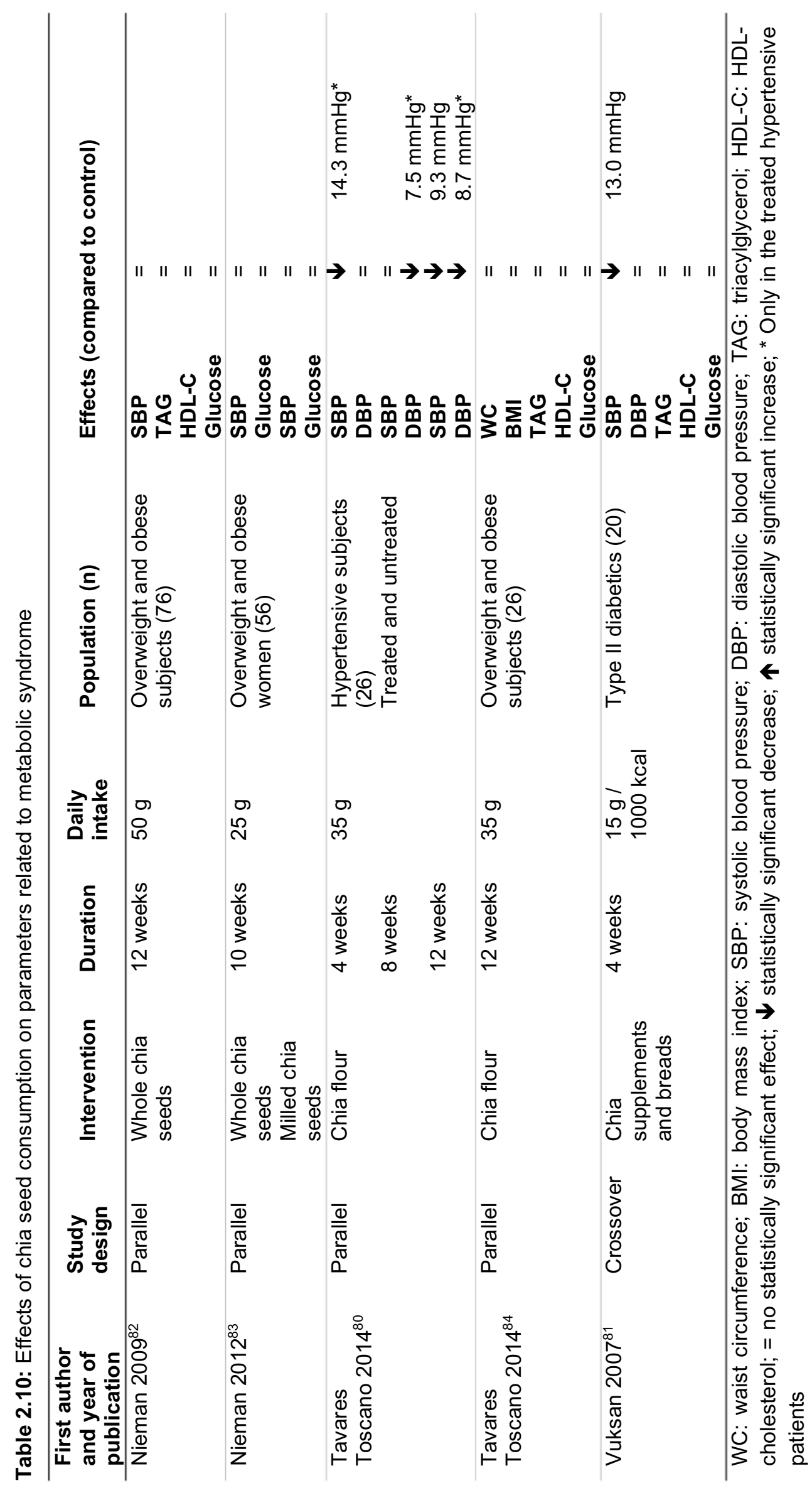




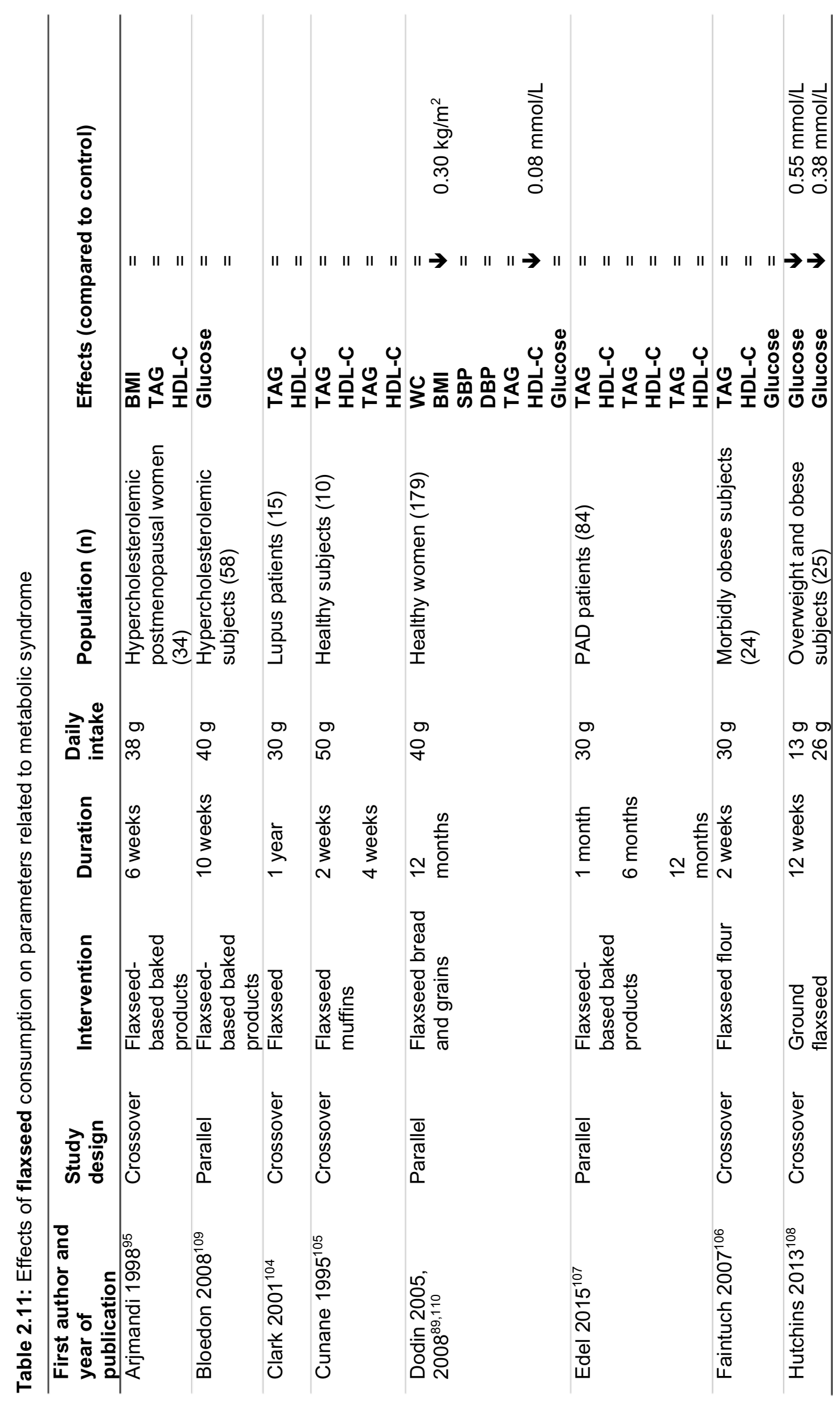




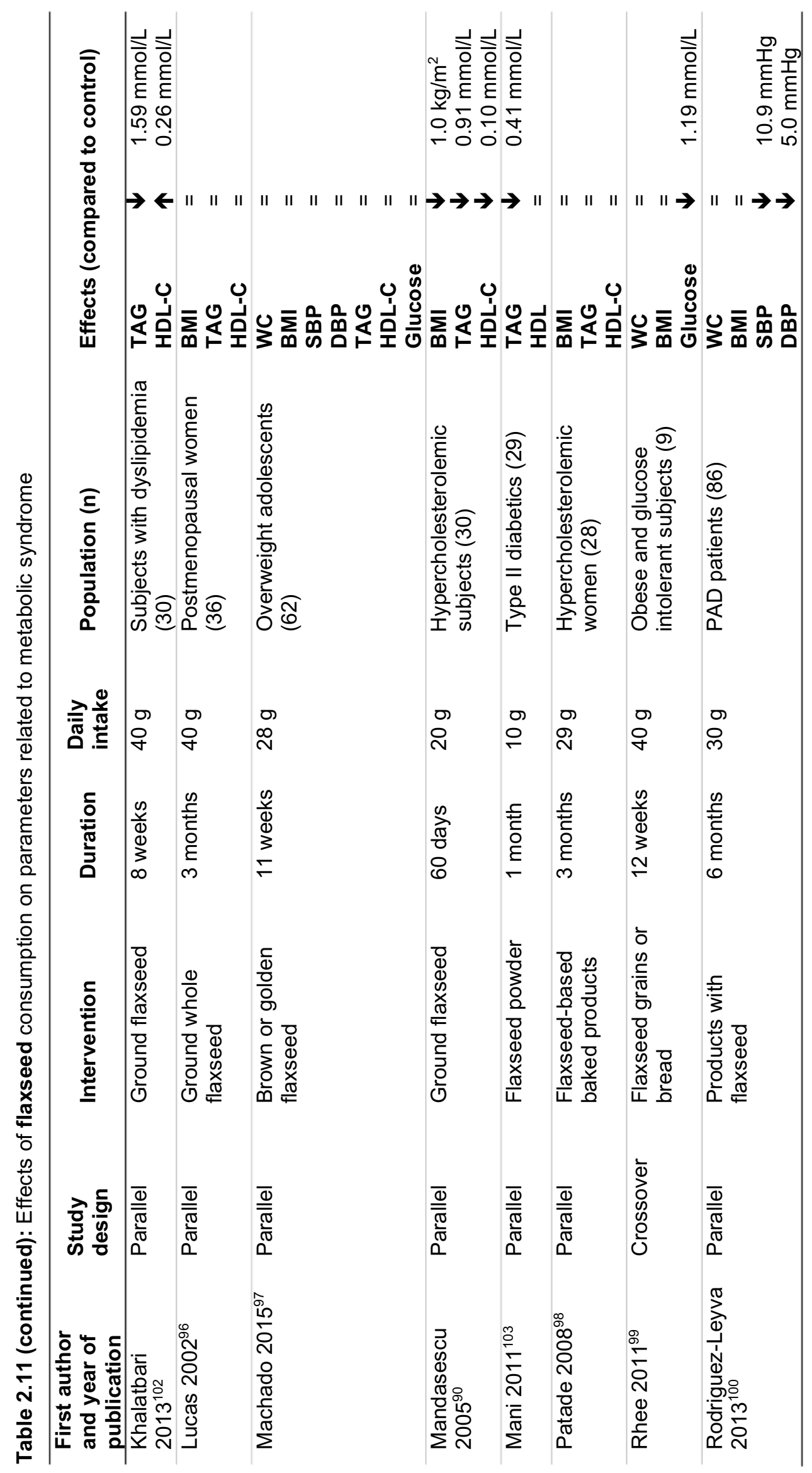




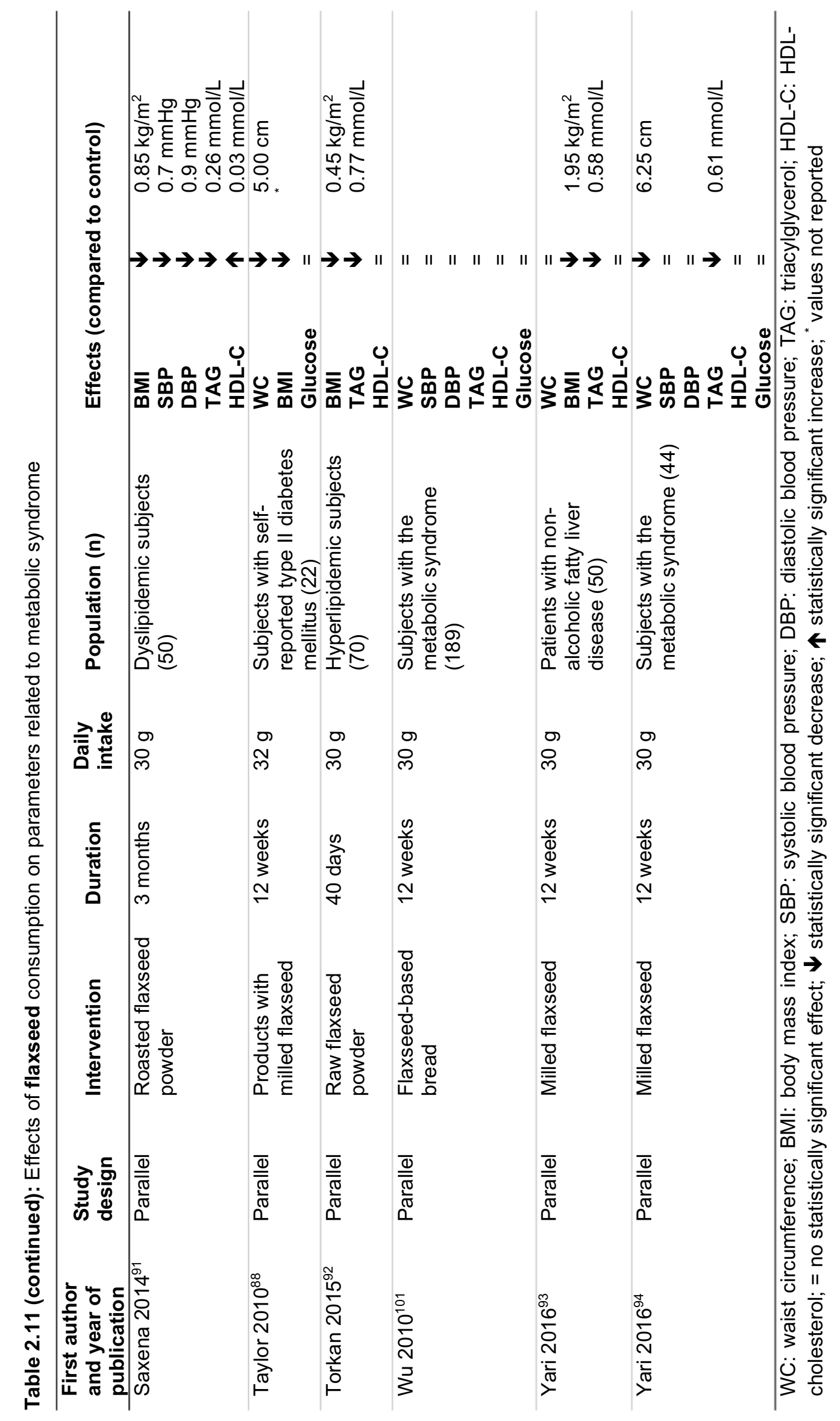




\section{Quinoa}

Last in the category seeds is quinoa. Quinoa (Chenopodium quinoa Willd.) is a grain originating from South America and the seeds are mostly used for consumption. ${ }^{111}$ It contains all essential amino acids, several minerals and vitamins, and is rich in linoleic acid.

In the only study identified (Table 2.12), quinoa consumption lowered BMI in postmenopausal women. ${ }^{112}$ Furthermore, triacylglycerol concentrations were lowered, whereas waist circumference, and concentrations of HDL cholesterol and glucose did not change.

\section{Cocoa}

Cocoa is harvested from the seeds of Theobroma cacao L. and used for the production of chocolate. Flavanols are highly abundant in cocoa, especially catechin and epicatechin. ${ }^{113}$

Of the sixteen studies identified (Table 2.13), systolic and diastolic blood pressure were measured in thirteen studies. In five of these studies, systolic and/or diastolic blood pressure was lowered, ${ }^{114-118}$ whereas blood pressure remained unchanged in the other eight studies. ${ }^{119-127}$ In one study with normocholesterolemic and hypercholesterolemic subjects, ${ }^{126} \mathrm{HDL}$ cholesterol concentrations increased in both groups. The same results were found in patients with stable congestive heart disease, ${ }^{121}$ subjects at high-risk for CVD, ${ }^{122,123}$ normal-weight and obese subjects, ${ }^{128}$ and in healthy men. ${ }^{119}$ However, HDL cholesterol concentrations were not changed in type II diabetic, ${ }^{129}$ in (pre-) hypertensive subjects, ${ }^{115-118,} 124$ in overweight and obese subjects, ${ }^{125,127}$ and in healthy men and women. ${ }^{114,130}$ Glucose concentrations were measured in eight studies, but lowered in only one study. ${ }^{126} \mathrm{BMI}$ increased in one study with subjects at high-risk for CVD, ${ }^{122,123}$ but remained stable in the other studies. ${ }^{115-118,124-127,129}$

\section{Maca}

The maca plant (Lepidium meyenii Walp.) originates from Peru, and its root and tuber provide relatively high amounts of essential amino acids and fibers. ${ }^{131}$ 

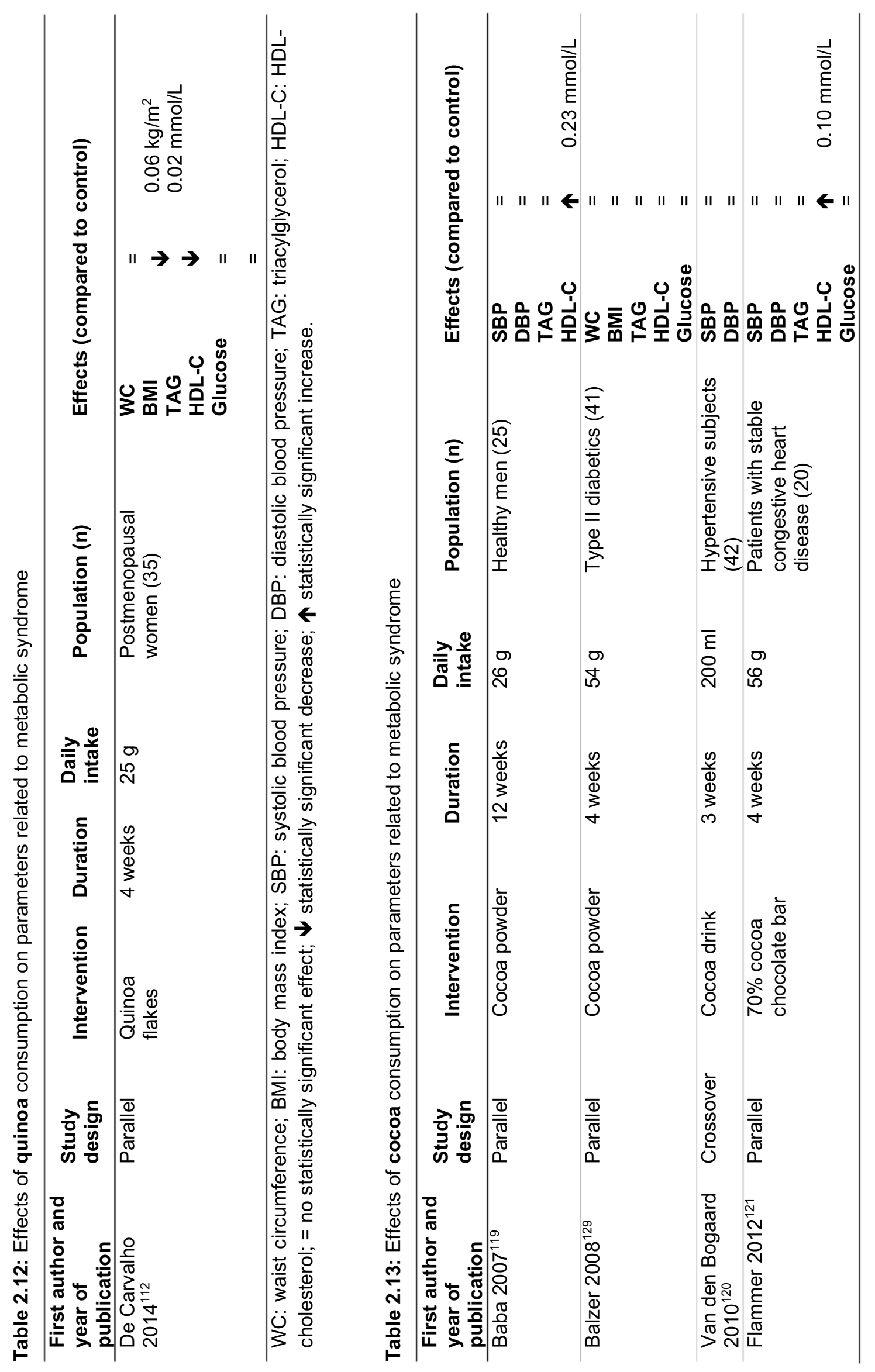


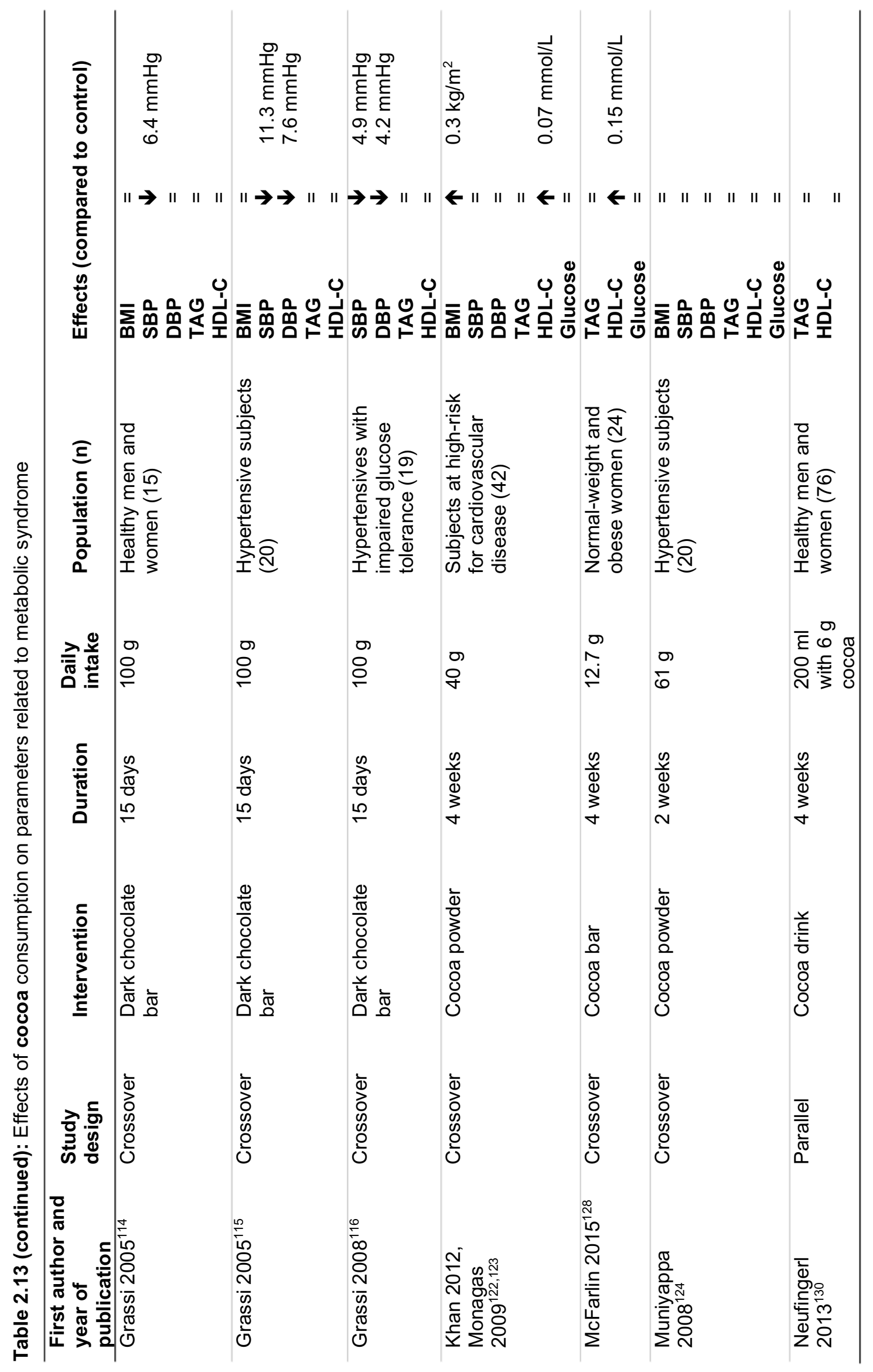




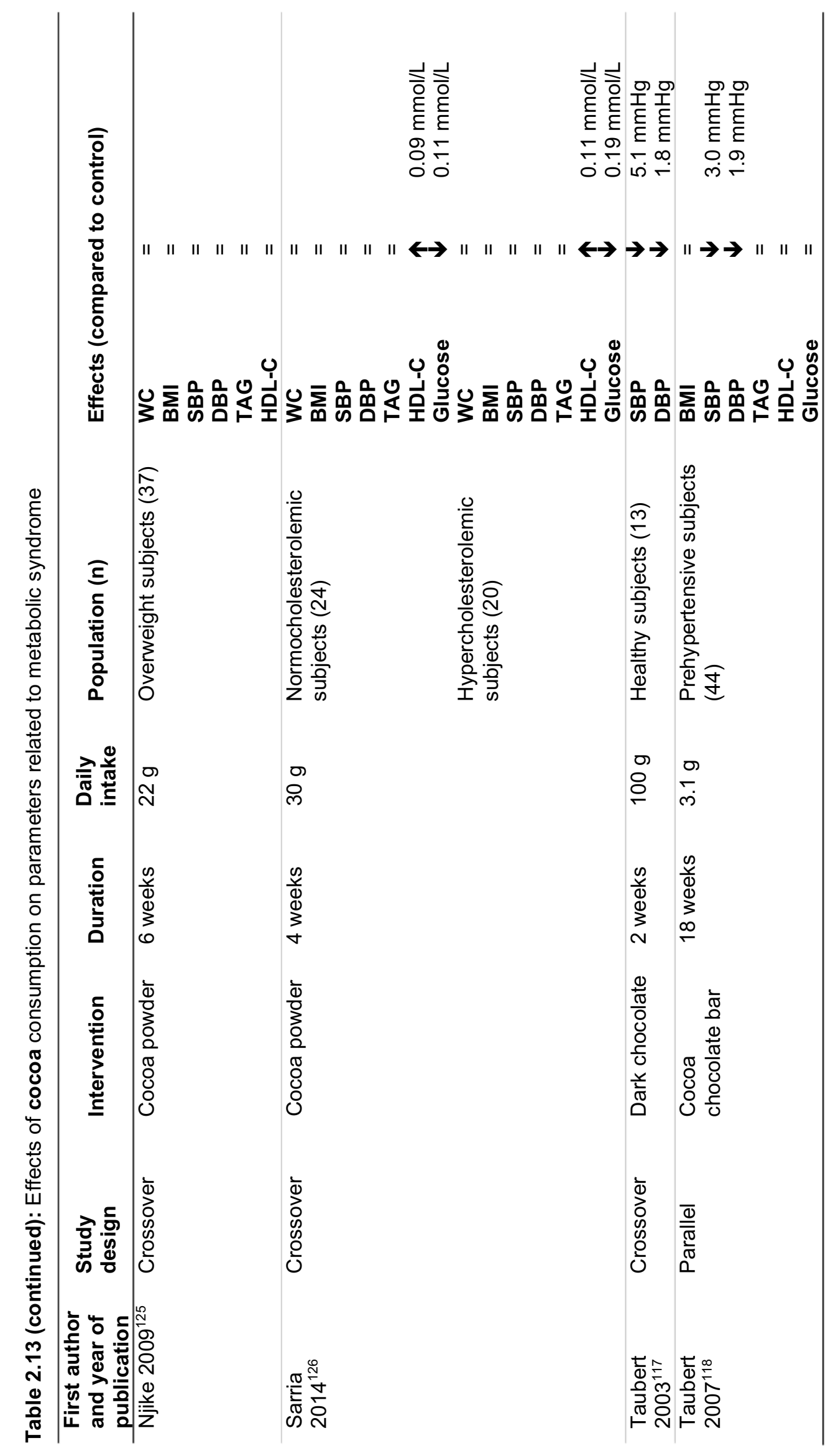




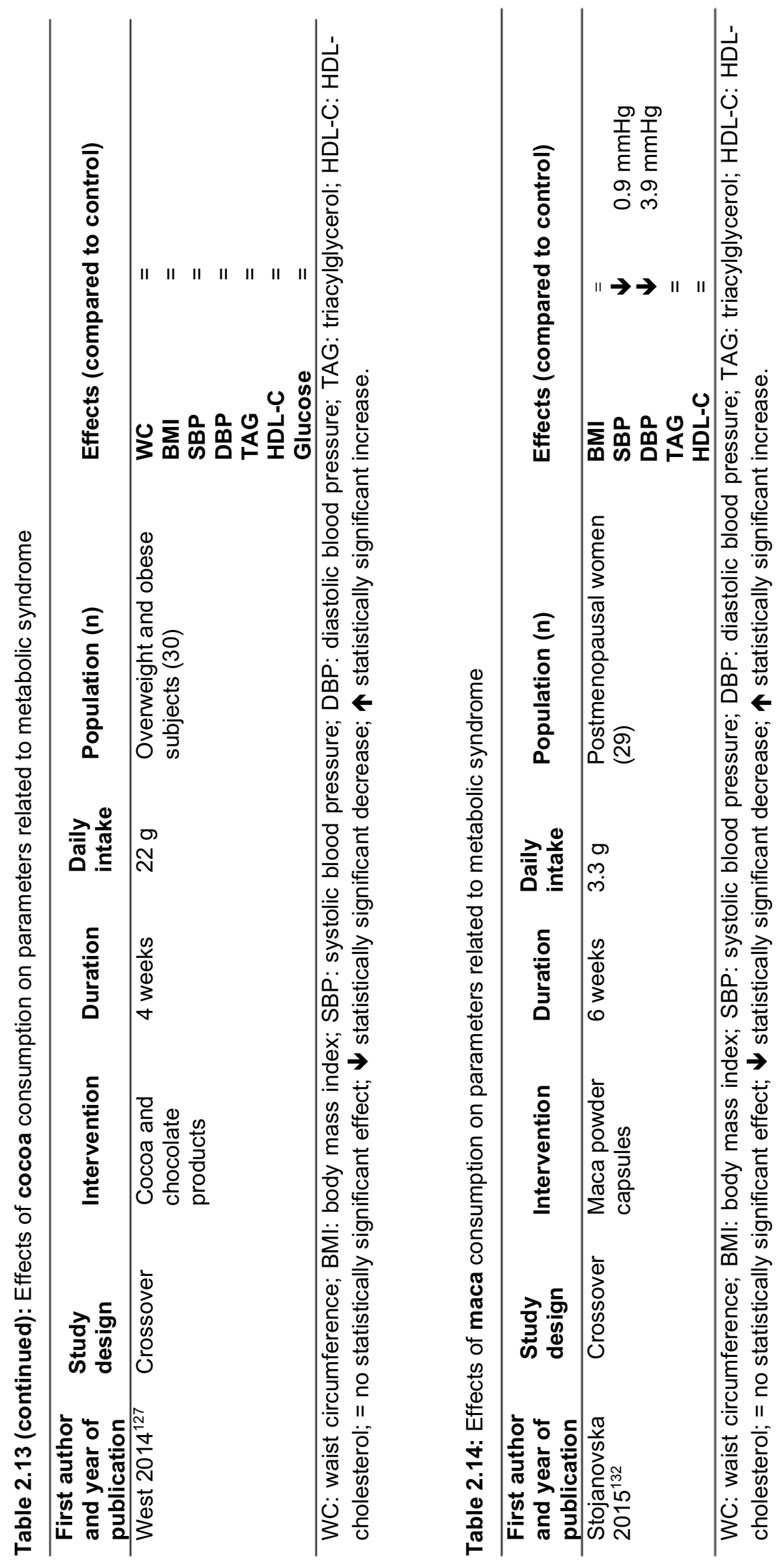


Only one study, carried out in postmenopausal women, was identified (Table 2.14). Maca powder consumption did not change $\mathrm{BMI}$, and triacylglycerol and $\mathrm{HDL}$ cholesterol concentrations, but decreased systolic and diastolic blood pressure. ${ }^{132}$

\section{Spirulina}

Spirulina (Arthrospira maxima (Setchell \& N.L.Gardner) Geitler, Arthrospira plantesis Gomont) is a blue-green algae containing carotenoids ( $\beta$-carotene and several xanthophylls), alpha-tocopherol, gamma-linoleic acid, and phycobiliproteins. ${ }^{133}$

Seven studies were identified (Table 2.15). In one study with hypertensive patients, BMI was significantly reduced after spirulina consumption. ${ }^{134}$ Results, however, were not confirmed in HIV patients ${ }^{135,136}$ and in children with the nephrotic syndrome. ${ }^{137}$ In the study of Miczke et al, ${ }^{134}$ systolic blood pressure was also decreased, while no effects were found on diastolic blood pressure. In contrast, Lee at al found that spirulina consumption in type II diabetics did not change systolic blood pressure, but decreased diastolic blood pressure. ${ }^{138}$ In addition, triacylglycerol concentrations were lowered in that study. Three studies in type II diabetics, ${ }^{139}$ hypercholesterolemic subjects ${ }^{140}$ and children with the nephrotic syndrome ${ }^{137}$ also found decreases in triacylglycerol concentrations. However, studies in elderly ${ }^{141}$ and HIV patients ${ }^{135,136}$ did not. HDL cholesterol concentrations were increased in HIVinfected patients ${ }^{135,136}$ and hypercholesterolemic subjects, ${ }^{140}$ but not in type II diabetics, ${ }^{138,139}$ elderly, ${ }^{141}$ and children with nephrotic syndrome. ${ }^{137}$ Three of the seven studies measured glucose concentrations, but no significant effects were found. ${ }^{135,136,138,139}$

\section{Wheatgrass}

Wheatgrass is harvested from wheat (Triticum aestivum L.) before it matures into whole cereal grain and is rich in chlorophyll. ${ }^{142}$

One study was identified (Table 2.16). In that study with hyperlipidemic women, consumption of wheatgrass powder lowered systolic and diastolic blood pressure, and triacylglycerol concentrations. ${ }^{143} \mathrm{HDL}$ cholesterol and glucose concentrations remained unchanged. 


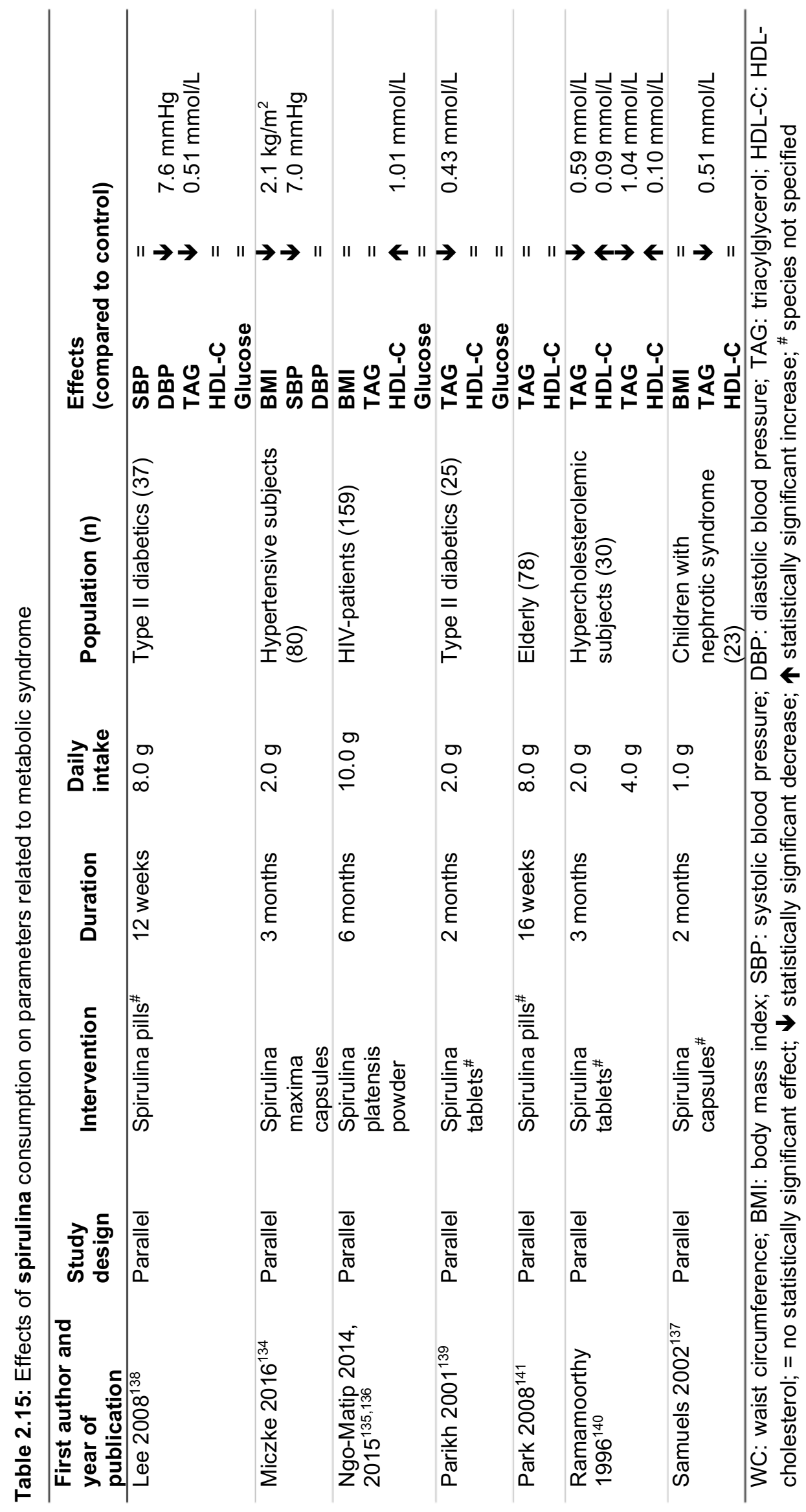




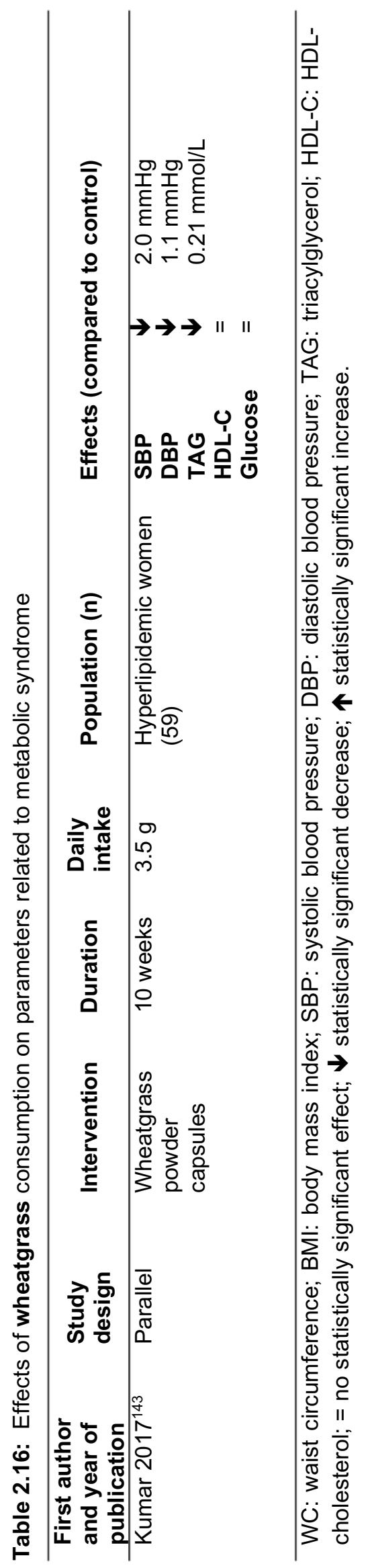




\section{Acai berries, hemp seed and bee pollen}

Acai (Euterpe oleracea Mart.) is a tree that is found in Central and South America. ${ }^{144}$ The berries of this tree contain flavonoids, mainly anthocyanins, and other phenolic compounds like catechin and isovitexin. ${ }^{144,145}$ The seeds of the plant Cannabis sativa L. are referred to as hemp seed and have no psychoactive properties. ${ }^{146,147} \mathrm{Hemp}$ seed is rich in polyunsaturated fatty acids, vitamins and minerals. Bee pollen are formed when honeybees collect pollen, which are merged together with nectar and honeybee enzymes. ${ }^{148}$ Bee pollen are rich in flavonoids (catechins and quercetin) and $\beta$-carotene, but the composition of bee pollen is variable and strongly depends on factors such as geographical location and season. No trials that matched the inclusion criteria were found for acai berries, hemp seed and bee pollen

\section{Discussion}

In this systematic review, studies evaluating effects of superfoods on parameters related to the metabolic syndrome have been critically evaluated. In general, a superfood is considered to be a nutrient-dense food, especially beneficial for health and well-being, ${ }^{149-151}$ for which no generally accepted definition exists. Therefore, several decisions had to be taken. First, the Internet was searched to make a broad selection of foods described as superfoods. Next, foods from groups that were already part of the dietary guidelines from the Netherlands were excluded because these foods are generally not positioned as superfoods. Some foods that can be considered as superfoods by others may therefore not have been included. It should further be noted that the perception of superfoods differs worldwide and is based on regional dietary habits. Acai berries and quinoa, for example, have been introduced only recently into the Western diet and are described as superfoods. However, these foods originate from South-America, where they are already part of the habitual diet for centuries ${ }^{111,147}$ and therefore not considered as superfoods in that part of the world. These considerations stress that the perception of a superfood differs between regions, which will make it even more difficult to agree upon a general and global accepted definition. 
Although we here focussed on the metabolic health effects of the 17 selected potential superfoods in human intervention trials, many of the selected foods - or extracts of these foods - have been investigated in numerous cell and animal studies. For example, flavonoids from berries (anthocyanins) or cocoa (catechin and epicatechin) have been shown to affect blood pressure in animal studies via nitric oxide-mediated pathways. ${ }^{152-154}$ Ginger extracts improved glucose concentrations in various animal models, such as type II diabetic $\mathrm{db} / \mathrm{db}$ mice and rats fed a high-fat diet, although other studies did not report any effects ${ }^{155}$ Cell studies suggested that these glucose-lowering effects were due to increased GLUT4-mediated glucose uptake in myocytes ${ }^{156}$ and insulin-dependent uptake in adipocytes. ${ }^{157}$ Lipid profiles have been improved by ginger, ${ }^{155}$ garlic extract, ${ }^{158}$ maca, ${ }^{159}$ spirulina, ${ }^{160}$ or wheatgrass ${ }^{161,162}$ in several animal models via different metabolic routes. Garlic extracts inhibited cholesterol synthesis in hepatocytes and HepG2 cells, ${ }^{163}$ whereas a spirulina extract reduced cholesterol absorption in the intestinal Caco-2 cell line. ${ }^{164}$ Wheatgrass, on the other hand, increased cholesterol excretion in hypercholesterolemic rats. ${ }^{161}$ Even though cell and animal studies were promising, there is overall only limited evidence in humans that the selected superfoods beneficially affect risk factors for the metabolic syndrome (Supplemental table 2.1). Most consistent are the results from the ginger trials, in which triacylglycerol and glucose concentrations were lowered in four out of five and five out of seven trials respectively. Despite the observed reductions in triacylglycerol concentrations, none of the four studies showed an effect on HDL cholesterol concentrations. This is somewhat unexpected, since HDL cholesterol and triacylglycerol concentrations are frequently inversely related due to cholesterylester transfer protein (CETP) activity. ${ }^{165}$ It should be noted, however, that diet-induced changes in these two lipids are not necessarily into opposite directions. ${ }^{166-168}$ Triacylglycerol concentrations were also affected by spirulina consumption in four out of six trials. These triacylglycerol-lowering effects were accompanied by increased HDL concentrations in one of these four trials. Garlic, flaxseed and cocoa consumption may have multiple effects on metabolic syndrome parameters, such as blood pressure and lipid profiles, but results are not consistent. For maca and wheatgrass, decreases in both systolic and diastolic blood pressure were observed, whereas triacylglycerol 
concentrations were lowered in studies with quinoa and wheatgrass consumption. However, as only one study for each of these superfoods was included, more studies are needed before any conclusions can be drawn.

To quantify effects, meta-analyses have been performed for some of the superfoods. In three meta-analyses, ${ }^{169-171}$ decreases in triacylglycerol and increases in HDL cholesterol concentrations were found after ginger and spirulina intake. Decreases in triacylglycerol concentrations were also evident from our results, but changes in HDL cholesterol not. No effects of cranberries or blueberries were found, ${ }^{172,173}$ while goji berry consumption decreased glucose concentrations in one meta-analysis. ${ }^{174}$ This decrease in glucose concentrations was not apparent from our results. We found no consistent effects on any of the parameters related to the metabolic syndrome of garlic, flaxseed and cocoa consumption. However, garlic consumption improved systolic and diastolic blood pressure, ${ }^{175}$ and concentrations of triacylglycerol, ${ }^{176} \mathrm{HDL}$ cholesterol ${ }^{177}$ and glucose ${ }^{178}$ in several meta-analyses. Also, flaxseed consumption improved waist circumference/BMI, ${ }^{179}$ and systolic and diastolic blood pressure in several meta-analyses, ${ }^{180,181}$ without affecting lipid concentrations. ${ }^{182}$ Systolic and/or diastolic blood pressure and lipid concentrations were improved after cocoa consumption. ${ }^{183-187}$ Thus, these meta-analyses reported more promising effects of goji berry, garlic, flaxseed and cocoa consumption on blood pressure and lipid profiles compared to our systematic review. Our conclusions were, however, based on statistical significance of the effects, while in meta-analyses a pooled estimate is calculated based on a weighted average from the results of the individual studies. Furthermore, heterogeneity was detected for most of the parameters studied in the meta-analyses. Subgroup analyses further revealed that population, ${ }^{169,171,175,177-179,182,183,185,186}$ duration of intervention, ${ }^{171,174,177-}$ 181,183-185 dose, ${ }^{171,184}$ and intervention type (whole food or extract) ${ }^{178-182}$ were associated with the efficacy of the interventions. In our review, we did not differentiate between these factors and we only included studies with whole foods, which may explain at least a part of the discrepancy in results. Well-controlled intervention trials specifically addressing these factors are needed to assess these discrepancies. 


\section{Limitations}

Studies in this review were selected based on predefined selection criteria, including the minimal duration of the intervention. Only trials that lasted at least 2 weeks were included, leading to the inclusion of studies with a median duration of 8 weeks (range from 2 to 52 weeks). Also, in the majority of the studies, subjects were asked not to change their habitual lifestyles, including energy intakes and exercise regimes. With this approach, it is possible to examine diet-induced effects on serum lipids, glucose and blood pressure, which may change and stabilize within a relatively short period. However, it is virtually impossible to detect changes in BMI and waist circumference in shorter-term studies.

Studies were also selected based on characteristics of the intervention product. Trials using isolated constituents from the selected superfoods were excluded. For example, studies that used isolated polysaccharides from goji berries or capsinoids from chili peppers as intervention products were not selected. However, examining effects of isolated compounds can provide insight into underlying mechanisms. In addition, if the effective compounds from a superfood are known, these can be isolated and used for the production of functional foods. On the other hand, the effects of the food matrix on for example absorption kinetics and interaction between components are not taken into account when isolated compounds are investigated. ${ }^{188}$

Lastly, it should be noted that this review focussed on the effects of foods labelled as superfoods on markers related to metabolic syndrome, and not on other potential health benefits. Conclusions from our review may be different if other potential health benefits are considered.

To summarize, a broadly accepted definition for superfoods is lacking and differences in the perception of superfoods around the world makes it challenging to agree upon a universally accepted definition. The superfoods show, based on the results of this review, only limited evidence for consistent effects on parameters related to metabolic syndrome. For many of the foods, presented results are contradictory or not convincing, while for other foods, the number of studies is limited. Results of several meta-analyses suggest that results might be influenced 
by various intervention-related factors, such as duration and dose of consumption of the included whole foods. To address these apparent discrepancies, well-controlled randomized trials specifically addressing these questions are needed. Even though the evidence for effects on metabolic syndrome parameters is limited, the selected foods might still have other potential health benefits. 


\section{References}

1. Alberti KG, Eckel RH, Grundy SM, Zimmet PZ, Cleeman JI, Donato KA, et al. Harmonizing the metabolic syndrome: a joint interim statement of the International Diabetes Federation Task Force on Epidemiology and Prevention; National Heart, Lung, and Blood Institute; American Heart Association; World Heart Federation; International Atherosclerosis Society; and International Association for the Study of Obesity. Circulation. 2009;120(16):1640-5

2. Brown L, Poudyal H, Panchal SK. Functional foods as potential therapeutic options for metabolic syndrome. Obes Rev. 2015;16(11):914-41.

3. De la Iglesia R, Loria-Kohen V, Zulet MA, Martinez JA, Reglero G, Ramirez de Molina A. Dietary strategies implicated in the prevention and treatment of metabolic syndrome. Int $\mathrm{J}$ Mol Sci. 2016;17(11).

4. Waltenberger B, Mocan A, Smejkal K, Heiss EH, Atanasov AG. Natural products to counteract the epidemic of cardiovascular and metabolic Disorder. Molecules. 2016;21.

5. Action E. Scientific concepts of functional foods in Europe: consensus document. $\mathrm{Br} \mathrm{J}$ Nutr. 1999;81(1):1-27.

6. Arai S. Global view on functional foods: Asian perspectives. Br J Nutr. 2002;88 Suppl 2:S139-43.

7. Health Council of the Netherlands, Dutch dietary guidelines, Health Council of the Netherlands, The Hague, 2015.

8. Michalska A, Łysiak G. Bioactive compounds of blueberries: Post-harvest factors influencing the nutritional value of products. Int J Mol Sci. 2015;16(8):18642-63.

9. McAnulty LS, Collier SR, Landram MJ, Whittaker DS, Isaacs SE, Klemka JM, et al. Six weeks daily ingestion of whole blueberry powder increases natural killer cell counts and reduces arterial stiffness in sedentary males and females. Nutr Res. 2014;34(7):577-84.

10. Riso P, Klimis-Zacas D, Bo C, Martini D, Campolo J, Vendrame S, et al. Effect of a wild blueberry (Vaccinium angustifolium) drink intervention on markers of oxidative stress, inflammation and endothelial function in humans with cardiovascular risk factors. Eur J Nutr. 2013; 52(3):949-61.

11. Basu A, Du M, Leyva MJ, Sanchez K, Betts NM, Wu M, et al. Blueberries decrease cardiovascular risk factors in obese men and women with metabolic syndrome. J Nutr. 2010;140(9):1582-7. .

12. Stull AJ, Cash KC, Champagne CM, Gupta AK, Boston R, Beyl RA, et al. Blueberries improve endothelial function, but not blood pressure, in adults with metabolic syndrome: a randomized, double-blind, placebo-controlled clinical trial. Nutrients. 2015;7(6):4107-23.

13. Johnson SA, Figueroa A, Navaei N, Wong A, Kalfon R, Ormsbee LT, et al. Daily blueberry consumption improves blood pressure and arterial stiffness in postmenopausal women with preand stage 1-hypertension: a randomized, double-blind, placebo-controlled clinical trial. J Acad Nutr Diet. 2015;115(3):369-77.

14. McAnulty SR, McAnulty LS, Morrow JD, Khardouni D, Shooter L, Monk J, et al. Effect of daily fruit ingestion on angiotensin converting enzyme activity, blood pressure, and oxidative stress in chronic smokers. Free Radical Res. 2005;39(11):1241-8. 
15. Stull AJ, Cash KC, Johnson WD, Champagne CM, Cefalu WT. Bioactives in blueberries improve insulin sensitivity in obese, insulin-resistant men and women. J Nutr. 2010;140(10):1764-8.

16. Nyberg S, Gerring E, Gjellan S, Vergara M, Lindstrom T, Nystrom FH. Effects of exercise with or without blueberries in the diet on cardio-metabolic risk factors: an exploratory pilot study in healthy subjects. Upsala J Med Sci. 2013;118(4):247-55.

17. Davidson E, Zimmermann BF, Jungfer E, Chrubasik-Hausmann S. Prevention of urinary tract infections with vaccinium products. Phytother Res. 2014;28(3):465-70.

18. Skrovankova S, Sumczynski D, Mlcek J, Jurikova T, Sochor J. Bioactive compounds and antioxidant activity in different types of berries. Int J Mol Sci. 2015;16(10):24673-706.

19. Novotny JA, Baer DJ, Khoo C, Gebauer SK, Charron CS. Cranberry juice consumption lowers markers of cardiometabolic risk, including blood pressure and circulating C-reactive protein, triglyceride, and glucose concentrations in adults. J Nutr. 2015;145(6):1185-93.

20. Duthie SJ, Jenkinson AM, Crozier A, Mullen W, Pirie L, Kyle J, et al. The effects of cranberry juice consumption on antioxidant status and biomarkers relating to heart disease and cancer in healthy human volunteers. Eur J Nutr. 2006;45(2):113-22.

21. Basu A, Betts NM, Ortiz J, Simmons B, Wu M, Lyons TJ. Low-energy cranberry juice decreases lipid oxidation and increases plasma antioxidant capacity in women with metabolic syndrome. Nutr Res. 2011;31(3):190-6.

22. Ruel G, Lapointe A, Pomerleau S, Couture P, Lemieux S, Lamarche B, et al. Evidence that cranberry juice may improve augmentation index in overweight men. Nutr Res. 2013;33(1):41-9.

23. Chambers BK, Camire ME. Can cranberry supplementation benefit adults with type 2 diabetes? Diabetes care. 2003;26(9):2695-6.

24. Lee IT, Chan YC, Lin CW, Lee WJ, Sheu WH. Effect of cranberry extracts on lipid profiles in subjects with Type 2 diabetes. Diabetic Med. 2008;25(12):1473-7.

25. Dohadwala MM, Holbrook M, Hamburg NM, Shenouda SM, Chung WB, Titas M, et al. Effects of cranberry juice consumption on vascular function in patients with coronary artery disease. Am $\mathrm{J}$ Clin Nutr. 2011;93(5):934-40.

26. Flammer AJ, Martin EA, GossI M, Widmer RJ, Lennon RJ, Sexton JA, et al. Polyphenol-rich cranberry juice has a neutral effect on endothelial function but decreases the fraction of osteocalcinexpressing endothelial progenitor cells. Eur J Nutr. 2013;52(1):289-96.

27. Potterat O. Goji (Lycium barbarum and L. chinense): Phytochemistry, pharmacology and safety in the perspective of traditional uses and recent popularity. Planta Med. 2010;76(1):7-19.

28. Amagase H, Nance DM. Lycium barbarum increases caloric expenditure and decreases waist circumference in healthy overweight men and women: pilot study. J Am Coll Nutr. 2011;30(5):3049.

29. Amagase H, Nance DM. A randomized, double-blind, placebo-controlled, clinical study of the general effects of a standardized Lycium barbarum (Goji) Juice, GoChi. J Altern Complement Med. 2008;14(4):403-12.

30. Amagase H, Sun B, Nance DM. Immunomodulatory effects of a standardized Lycium barbarum fruit juice in Chinese older healthy human subjects. J Med Food. 2009;12(5):1159-65. 
31. Giampieri F, Forbes-Hernandez TY, Gasparrini M, Alvarez-Suarez JM, Afrin S, Bompadre S, et al. Strawberry as a health promoter: an evidence based review. Food Funct. 2015;6(5):1386-98.

32. Basu A, Betts NM, Nguyen A, Newman ED, Fu D, Lyons TJ. Freeze-dried strawberries lower serum cholesterol and lipid peroxidation in adults with abdominal adiposity and elevated serum lipids. The J Nutr. 2014;144(6):830-7.

33. Basu A, Fu DX, Wilkinson M, Simmons B, Wu M, Betts NM, et al. Strawberries decrease atherosclerotic markers in subjects with metabolic syndrome. Nutr Res. 2010;30(7):462-9.

34. Jenkins DJ, Nguyen TH, Kendall CW, Faulkner DA, Bashyam B, Kim IJ, et al. The effect of strawberries in a cholesterol-lowering dietary portfolio. Metab Clin Exp. 2008;57(12):1636-44.

35. Burton-Freeman B, Linares A, Hyson D, Kappagoda T. Strawberry modulates LDL oxidation and postprandial lipemia in response to high-fat meal in overweight hyperlipidemic men and women. $J$ Am Coll Nutr. 2010;29(1):46-54.

36. Zunino SJ, Parelman MA, Freytag TL, Stephensen CB, Kelley DS, Mackey BE, et al. Effects of dietary strawberry powder on blood lipids and inflammatory markers in obese human subjects. $\mathrm{Br}$ J Nutr. 2012;108(5):900-9.

37. Ellis CL, Edirisinghe I, Kappagoda T, Burton-Freeman B. Attenuation of meal-induced inflammatory and thrombotic responses in overweight men and women after 6-week daily strawberry (Fragaria) intake. A randomized placebo-controlled trial. J Atheroscler Thromb. 2011;18(4):318-27.

38. Moazen S, Amani R, Homayouni Rad A, Shahbazian H, Ahmadi K, Taha Jalali M. Effects of freezedried strawberry supplementation on metabolic biomarkers of atherosclerosis in subjects with type 2 diabetes: a randomized double-blind controlled trial. Ann Nutr Metab. 2013;63(3):256-64.

39. Asnin L, Park SW. Isolation and analysis of bioactive compounds in Capsicum peppers. Crit Rev Food Sci Nutr. 2015;55(2):254-89.

40. Ahuja KD, Ball MJ. Effects of daily ingestion of chilli on serum lipoprotein oxidation in adult men and women. Br J Nutr. 2006;96(2):239-42.

41. Ahuja KD, Robertson IK, Geraghty DP, Ball MJ. Effects of chili consumption on postprandial glucose, insulin, and energy metabolism. Am J Clin Nutr. 2006;84(1):63-9.

42. Ahuja KD, Robertson IK, Geraghty DP, Ball MJ. The effect of 4-week chilli supplementation on metabolic and arterial function in humans. Eur J Clin Nutr. 2007;61(3):326-33.

43. Nieman DC, Cialdella-Kam L, Knab AM, Shanely RA. Influence of red pepper spice and turmeric on inflammation and oxidative stress biomarkers in overweight females: a metabolomics approach. Plant Foods Hum Nutr. 2012;67(4):415-21.

44. Martins N, Petropoulos S, Ferreira IC. Chemical composition and bioactive compounds of garlic (Allium sativum L.) as affected by pre- and post-harvest conditions: A review. Food Chem. 2016;211:41-50.

45. Ashraf R, Khan RA, Ashraf I, Qureshi AA. Effects of Allium sativum (garlic) on systolic and diastolic blood pressure in patients with essential hypertension. Pak J Pharm Sci 2013;26(5):859-63.

46. Kiesewetter $H$, Jung F, Jung EM, Blume J, Mrowietz C, Birk A, et al. Effects of garlic coated tablets in peripheral arterial occlusive disease. J Clin Invest. 1993;71(5):383-6. 
47. Kiesewetter H, Jung F, Jung EM, Mroweitz C, Koscielny J, Wenzel E. Effect of garlic on platelet aggregation in patients with increased risk of juvenile ischaemic attack. Eur $\mathrm{J}$ Clin Pharmacol. 1993;45(4):333-6.

48. Isaacsohn JL, Moser M, Stein EA, Dudley K, Davey JA, Liskov E, et al. Garlic powder and plasma lipids and lipoproteins: a multicenter, randomized, placebo-controlled trial. Arch Intern Med. 1998;158(11):1189-94.

49. Jain AK, Vargas R, Gotzkowsky S, McMahon FG. Can garlic reduce levels of serum lipids? A controlled clinical study. Am J Med. 1993;94(6):632-5.

50. McCrindle BW, Helden E, Conner WT. Garlic extract therapy in children with hypercholesterolemia. JAMA Pediatr. 1998;152(11):1089-94.

51. Simons LA, Balasubramaniam S, Konigsmark M, Parfitt A, Simons J, Peters W. On the effect of garlic on plasma lipids and lipoproteins in mild hypercholesterolaemia. Atherosclerosis. 1995; $113(2): 219-25$.

52. Aalami-Harandi R, Karamali M, Asemi Z. The favorable effects of garlic intake on metabolic profiles, hs-CRP, biomarkers of oxidative stress and pregnancy outcomes in pregnant women at risk for pre-eclampsia: randomized, double-blind, placebo-controlled trial. J Metern Fetal Neonatal Med : the official journal of the European Association of Perinatal Medicine, the Federation of Asia and Oceania Perinatal Societies, the International Society of Perinatal Obstet. 2015;28(17):2020-7.

53. Ziaei S, Hantoshzadeh S, Rezasoltani P, Lamyian M. The effect of garlic tablet on plasma lipids and platelet aggregation in nulliparous pregnants at high risk of preeclampsia. Eur $\mathrm{J}$ Obestet Gynecol Reprod Biol 2001;99(2):201-6.

54. Mahdavi-Roshan M, Zahedmehr A, Mohammad-Zadeh A, Sanati HR, Shakerian F, Firouzi A, et al. Effect of garlic powder tablet on carotid intima-media thickness in patients with coronary artery disease: a preliminary randomized controlled trial. Nutr Health. 2013;22(2):143-55.

55. Turner B, Molgaard C, Marckmann P. Effect of garlic (Allium sativum) powder tablets on serum lipids, blood pressure and arterial stiffness in normo-lipidaemic volunteers: a randomised, doubleblind, placebo-controlled trial. Br J Nutr. 2004;92(4):701-6.

56. Ernst E, Weihmayr T, Matrai A. Garlic and blood lipids. Br Med J. 1985;291(6488):139.

57. Ashraf R, Khan RA, Ashraf I. Garlic (Allium sativum) supplementation with standard antidiabetic agent provides better diabetic control in type 2 diabetes patients. Pak J Pharm Sci. 2011;24(4):56570 .

58. Adler AJ, Holub BJ. Effect of garlic and fish-oil supplementation on serum lipid and lipoprotein concentrations in hypercholesterolemic men. Am J Clin Nutr. 1997;65(2):445-50.

59. Gardner CD, Chatterjee LM, Carlson JJ. The effect of a garlic preparation on plasma lipid levels in moderately hypercholesterolemic adults. Atherosclerosis. 2001;154(1):213-20

60. Gardner CD, Lawson LD, Block E, Chatterjee LM, Kiazand A, Balise RR, et al. Effect of raw garlic vs commercial garlic supplements on plasma lipid concentrations in adults with moderate hypercholesterolemia: a randomized clinical trial. Arch Intern Med. 2007;167(4):346-53.

61. Kojuri J, Vosoughi AR, Akrami M. Effects of anethum graveolens and garlic on lipid profile in hyperlipidemic patients. Lipids Health Dis. 2007;6:5. 
62. Neil HA, Silagy CA, Lancaster T, Hodgeman J, Vos K, Moore JW, et al. Garlic powder in the treatment of moderate hyperlipidaemia: a controlled trial and meta-analysis. Clin Med. 1996;30(4):329-34.

63. Superko HR, Krauss RM. Garlic powder, effect on plasma lipids, postprandial lipemia, low-density lipoprotein particle size, high-density lipoprotein subclass distribution and lipoprotein(a). J Am Coll Cardiol. 2000;35(2):321-6.

64. Ashraf R, Aamir K, Shaikh AR, Ahmed T. Effects of garlic on dyslipidemia in patients with type 2 diabetes mellitus. J Ayub Med Coll Abbottabad. 2005;17(3):60-4.

65. van Doorn MB, Espirito Santo SM, Meijer P, Kamerling IM, Schoemaker RC, Dirsch V, et al. Effect of garlic powder on C-reactive protein and plasma lipids in overweight and smoking subjects. Am J Clin Nutr. 2006;84(6):1324-9.

66. Prasad S, Tyagi AK. Ginger and its constituents: role in prevention and treatment of gastrointestinal cancer. Gastroenterol Res Pract. 2015;2015:142979.

67. Rahmani AH, Shabrmi FM, Aly SM. Active ingredients of ginger as potential candidates in the prevention and treatment of diseases via modulation of biological activities. Int $\mathrm{J}$ Physiol Pathophysiol Pharmacol. 2014;6(2):125-36.

68. Alizadeh-Navaei R, Roozbeh F, Saravi M, Pouramir M, Jalali F, Moghadamnia AA. Investigation of the effect of ginger on the lipid levels. A double blind controlled clinical trial. Saudi Med J. 2008;29(9):1280-4.

69. Arablou T, Aryaeian N, Valizadeh M, Sharifi F, Hosseini A, Djalali M. The effect of ginger consumption on glycemic status, lipid profile and some inflammatory markers in patients with type 2 diabetes mellitus. Int J Food Sci Nutr. 2014;65(4):515-20.

70. Mahluji S, Attari VE, Mobasseri M, Payahoo L, Ostadrahimi A, Golzari SE. Effects of ginger (Zingiber officinale) on plasma glucose level, $\mathrm{HbA} 1 \mathrm{c}$ and insulin sensitivity in type 2 diabetic patients. Int J Food Sci Nutr. 2013;64(6):682-6.

71. Tabibi H, Imani H, Atabak S, Najafi I, Hedayati M, Rahmani L. Effects of ginger on serum lipids and lipoproteins in peritoneal dialysis patients: a randomized controlled trial. Peritoneal Dial Int. 2016;36(2):140-5.

72. Imani H, Tabibi H, Najafi I, Atabak S, Hedayati M, Rahmani L. Effects of ginger on serum glucose, advanced glycation end products, and inflammation in peritoneal dialysis patients. Nutr. 2015;31(5):703-7.

73. Bordia A, Verma SK, Srivastava KC. Effect of ginger (Zingiber officinale Rosc.) and fenugreek (Trigonella foenumgraecum L.) on blood lipids, blood sugar and platelet aggregation in patients with coronary artery disease. Prostaglandins Leukot Essent Fatty Acids. 1997;56(5):379-84.

74. Mozaffari-Khosravi H, Talaei B, Jalali BA, Najarzadeh A, Mozayan MR. The effect of ginger powder supplementation on insulin resistance and glycemic indices in patients with type 2 diabetes: a randomized, double-blind, placebo-controlled trial. Complement Ther Med. 2014;22(1):9-16.

75. Ebrahimzadeh Attari V, Asghari Jafarabadi M, Zemestani M, Ostadrahimi A. Effect of Zingiber officinale Supplementation on Obesity Management with Respect to the Uncoupling Protein 1 - 
3826A>G and ss3-adrenergic Receptor Trp64Arg Polymorphism. Phytother Res. 2015;29(7):10329.

76. Ebrahimzadeh Attari V, Ostadrahimi A, Asghari Jafarabadi M, Mehralizadeh S, Mahluji S. Changes of serum adipocytokines and body weight following Zingiber officinale supplementation in obese women: a RCT. Eur J Nutr. 2016;55(6):2129-36.

77. Shidfar F, Rajab A, Rahideh T, Khandouzi N, Hosseini S, Shidfar S. The effect of ginger (Zingiber officinale) on glycemic markers in patients with type 2 diabetes. J Comlementary Integr Med. 2015;12(2):165-70.

78. Azimi P, Ghiasvand R, Feizi A, Hosseinzadeh J, Bahreynian M, Hariri M, et al. Effect of cinnamon, cardamom, saffron and ginger consumption on blood pressure and a marker of endothelial function in patients with type 2 diabetes mellitus: A randomized controlled clinical trial. Blood pressure. 2016;25(3):133-40.

79. Muñoz LA, Cobos A, Diaz O, Aguilera JM. Chia seed (Salvia hispanica): an ancient grain and a new functional food. Food Rev Int. 2013;29(4):394-408.

80. Toscano LT, da Silva CS, Toscano LT, de Almeida AE, Santos Ada C, Silva AS. Chia flour supplementation reduces blood pressure in hypertensive subjects. Plant Foods Hum Nutr. 2014;69(4):392-8.

81. Vuksan V, Whitham D, Sievenpiper JL, Jenkins AL, Rogovik AL, Bazinet RP, et al. Supplementation of conventional therapy with the novel grain Salba (Salvia hispanica L.) improves major and emerging cardiovascular risk factors in type 2 diabetes: results of a randomized controlled trial. Diabetes care. 2007;30(11):2804-10.

82. Nieman DC, Cayea EJ, Austin MD, Henson DA, McAnulty SR, Jin F. Chia seed does not promote weight loss or alter disease risk factors in overweight adults. Nutr Res. 2009;29(6):414-8.

83. Nieman DC, Gillitt N, Jin F, Henson DA, Kennerly K, Shanely RA, et al. Chia seed supplementation and disease risk factors in overweight women: a metabolomics investigation. J Altern Complement Med. 2012;18(7):700-8.

84. Tavares Toscano L, Tavares Toscano L, Leite Tavares R, da Oliveira Silva CS, Silva AS. Chia induces clinically discrete weight loss and improves lipid profile only in altered previous values. Nutr Hosp. 2014;31(3):1176-82.

85. Kajla P, Sharma A, Sood DR. Flaxseed-a potential functional food source. J Food Sci Technol. 2015;52(4):1857-71.

86. Dubois V, Breton S, Linder M, Fanni J, Parmentier M. Fatty acid profiles of 80 vegetable oils with regard to their nutritional potential. Eur J Lipid Sci Technol. 2007;109(7):710-32.

87. Touré A, Xueming X. Flaxseed lignans: source, biosynthesis, metabolism, antioxidant activity, bioactive components, and health benefits. Compr Rev Food Sci Food Saf. 2010;9(3):261-9.

88. Taylor CG, Noto AD, Stringer DM, Froese S, Malcolmson L. Dietary milled flaxseed and flaxseed oil improve $\mathrm{N}-3$ fatty acid status and do not affect glycemic control in individuals with well-controlled type 2 diabetes. J Am Coll Nutr. 2010;29(1):72-80.

89. Dodin S, Lemay A, Jacques H, Legare F, Forest JC, Masse B. The effects of flaxseed dietary supplement on lipid profile, bone mineral density, and symptoms in menopausal women: a 
randomized, double-blind, wheat germ placebo-controlled clinical trial. J Clin Endocrinol Metab. 2005;90(3):1390-7.

90. Mandasescu S, Mocanu V, Dascalita AM, Haliga R, Nestian I, Stitt PA, et al. Flaxseed supplementation in hyperlipidemic patients. Rev Med Chir Soc Med Nat lasi. 2005;109(3):502-6.

91. Saxena $S$, Katare $C$. Evaluation of flaxseed formulation as a potential therapeutic agent in mitigation of dyslipidemia. Biomed J. 2014;37(6):386-90.

92. Torkan M, Entezari MH, Siavash M. Effect of flaxseed on blood lipid level in hyperlipidemic patients. Rev Recent Clin Trials. 2015;10(1):61-7.

93. Yari Z, Rahimlou M, Eslamparast T, Ebrahimi-Daryani N, Poustchi H, Hekmatdoost A. Flaxseed supplementation in non-alcoholic fatty liver disease: a pilot randomized, open labeled, controlled study. Int J Food Sci Nutr. 2016;67(4):461-9.

94. Yari Z, Rahimlou M, Poustchi H, Hekmatdoost A. Flaxseed supplementation in metabolic syndrome management: a pilot randomized, open-labeled, controlled study. Phytother Res. 2016;30(8):133944.

95. Arjmandi $\mathrm{BH}$, Khan DA, Juma S, Drum ML, Venkatesh $\mathrm{S}$, Sohn E, et al. Whole flaxseed consumption lowers serum LDL-cholesterol and lipoprotein (a) concentrations in postmenopausal women. Nutr Res. 1998;18(7):1203-14.

96. Lucas EA, Wild RD, Hammond LJ, Khalil DA, Juma S, Daggy BP, et al. Flaxseed improves lipid profile without altering biomarkers of bone metabolism in postmenopausal women. J Clin Endocrinol Metab. 2002;87(4):1527-32.

97. Machado AM, de Paula H, Cardoso LD, Costa NM. Effects of brown and golden flaxseed on the lipid profile, glycemia, inflammatory biomarkers, blood pressure and body composition in overweight adolescents. Nutrition (Burbank, Los Angeles County, Calif). 2015;31(1):90-6.

98. Patade A, Devareddy L, Lucas EA, Korlagunta K, Daggy BP, Arjmandi BH. Flaxseed reduces total and LDL cholesterol concentrations in Native American postmenopausal women. J Women Health. 2008;17(3):355-66.

99. Rhee $\mathrm{Y}$, Brunt A. Flaxseed supplementation improved insulin resistance in obese glucose intolerant people: a randomized crossover design. Nutr J. 2011;10:44.

100. Rodriguez-Leyva D, Weighell W, Edel AL, LaVallee R, Dibrov E, Pinneker R, et al. Potent antihypertensive action of dietary flaxseed in hypertensive patients. Hypertension (Dallas, Tex : 1979). 2013;62(6):1081-9.

101. Wu H, Pan A, Yu Z, Qi Q, Lu L, Zhang G, et al. Lifestyle counseling and supplementation with flaxseed or walnuts influence the management of metabolic syndrome. J Nutr. 2010;140(11):193742.

102. Khalatbari Soltani S, Jamaluddin R, Tabibi H, Mohd Yusof BN, Atabak S, Loh SP, et al. Effects of flaxseed consumption on systemic inflammation and serum lipid profile in hemodialysis patients with lipid abnormalities. Hemodial Int. 2013;17(2):275-81.

103. Mani UV, Mani I, Biswas M, Kumar SN. An open-label study on the effect of flax seed powder (Linum usitatissimum) supplementation in the management of diabetes mellitus. J Diet Suppl. 2011;8(3):257-65. 
104. Clark WF, Kortas C, Heidenheim AP, Garland J, Spanner E, Parbtani A. Flaxseed in lupus nephritis: a two-year nonplacebo-controlled crossover study. J Am Coll Nutr. 2001;20(2 Suppl):143-8.

105. Cunnane SC, Hamadeh MJ, Liede AC, Thompson LU, Wolever TM, Jenkins DJ. Nutritional attributes of traditional flaxseed in healthy young adults. Am J Clin Nutr. 1995;61(1):62-8

106. Faintuch J, Horie LM, Barbeiro HV, Barbeiro DF, Soriano FG, Ishida RK, et al. Systemic inflammation in morbidly obese subjects: response to oral supplementation with alpha-linolenic acid. Obes Surg. 2007;17(3):341-7.

107. Edel AL, Rodriguez-Leyva D, Maddaford TG, Caligiuri SP, Austria JA, Weighell W, et al. Dietary flaxseed independently lowers circulating cholesterol and lowers it beyond the effects of cholesterol-lowering medications alone in patients with peripheral artery disease. J Nutr. 2015;145(4):749-57.

108. Hutchins AM, Brown BD, Cunnane SC, Domitrovich SG, Adams ER, Bobowiec CE. Daily flaxseed consumption improves glycemic control in obese men and women with pre-diabetes: a randomized study. Nutr Res. 2013;33(5):367-75.

109. Bloedon LT, Balikai S, Chittams J, Cunnane SC, Berlin JA, Rader DJ, et al. Flaxseed and cardiovascular risk factors: results from a double blind, randomized, controlled clinical trial. J Am Coll Nutr. 2008;27(1):65-74.

110. Dodin S, Cunnane SC, Masse B, Lemay A, Jacques H, Asselin G, et al. Flaxseed on cardiovascular disease markers in healthy menopausal women: a randomized, double-blind, placebo-controlled trial. Nutrition. 2008;24(1):23-30.

111. Vega-Galvez A, Miranda M, Vergara J, Uribe E, Puente L, Martinez EA. Nutrition facts and functional potential of quinoa (Chenopodium quinoa willd.), an ancient Andean grain: a review. J Sci Food Agric. 2010;90(15):2541-7.

112. Carvalho FG, Ovídio PP, Padovan GJ, Jordão Junior AA, Marchini JS, Navarro AM. Metabolic parameters of postmenopausal women after quinoa or corn flakes intake--a prospective and double-blind study. Int J Food Sci Nutr. 2014; 65(3):380-5.

113. Belščak A, Komes D, Horžić $D$, Ganić KK, Karlović D. Comparative study of commercially available cocoa products in terms of their bioactive composition. Food Res Int. 2009;42(5):707-16.

114. Grassi D, Lippi C, Necozione S, Desideri G, Ferri C. Short-term administration of dark chocolate is followed by a significant increase in insulin sensitivity and a decrease in blood pressure in healthy persons. Am J Clin Nutr. 2005;81(3):611-4.

115. Grassi D, Necozione S, Lippi C, Croce G, Valeri L, Pasqualetti P, et al. Cocoa reduces blood pressure and insulin resistance and improves endothelium-dependent vasodilation in hypertensives. Hypertension. 2005; 46(2):398-405.

116. Grassi D, Desideri G, Necozione S, Lippi C, Casale R, Properzi G, et al. Blood pressure is reduced and insulin sensitivity increased in glucose-intolerant, hypertensive subjects after 15 days of consuming high-polyphenol dark chocolate. J Nutr. 2008;138(9):1671-6.

117. Taubert $D$, Berkels R, Roesen R, Klaus W. Chocolate and blood pressure in elderly individuals with isolated systolic hypertension. JAMA. 2003;290(8):1029-30. 
118. Taubert D, Roesen R, Lehmann C, Jung N, Schomig E. Effects of low habitual cocoa intake on blood pressure and bioactive nitric oxide: a randomized controlled trial. Jama. 2007;298(1):49-60.

119. Baba S, Osakabe N, Kato Y, Natsume M, Yasuda A, Kido T, et al. Continuous intake of polyphenolic compounds containing cocoa powder reduces LDL oxidative susceptibility and has beneficial effects on plasma HDL-cholesterol concentrations in humans. Am J Clin Nutr. 2007;85(3):709-17.

120. Bogaard B, Draijer R, Westerhof BE, Meiracker AH, Montfrans GA, Born BJ. Effects on peripheral and central blood pressure of cocoa with natural or high-dose theobromine: a randomized, doubleblind crossover trial. Hypertension. 2010; 56(5):839-46.

121. Flammer AJ, Sudano I, Wolfrum M, Thomas R, Enseleit F, Periat D, et al. Cardiovascular effects of flavanol-rich chocolate in patients with heart failure. Eur Heart J. 2012;33(17):2172-80.

122. Khan N, Monagas M, Andres-Lacueva C, Casas R, Urpi-Sarda M, Lamuela-Raventos RM, et al. Regular consumption of cocoa powder with milk increases HDL cholesterol and reduces oxidized LDL levels in subjects at high-risk of cardiovascular disease. Nutr Metab Cardiovasc Dis. 2012;22(12):1046-53.

123. Monagas M, Khan N, Andres-Lacueva C, Casas R, Urpi-Sarda M, Llorach R, et al. Effect of cocoa powder on the modulation of inflammatory biomarkers in patients at high risk of cardiovascular disease. Am J Clin Nutr. 2009;90(5):1144-50.

124. Muniyappa R, Hall G, Kolodziej TL, Karne RJ, Crandon SK, Quon MJ. Cocoa consumption for 2 wk enhances insulin-mediated vasodilatation without improving blood pressure or insulin resistance in essential hypertension. Am J Clin Nutr. 2008;88(6):1685-96.

125. Njike VY, Faridi Z, Shuval K, Dutta S, Kay CD, West SG, et al. Effects of sugar-sweetened and sugar-free cocoa on endothelial function in overweight adults. Int J Cardiol. 2011;149(1):83-8.

126. Sarria B, Martinez-Lopez S, Sierra-Cinos JL, Garcia-Diz L, Mateos R, Bravo L. Regular consumption of a cocoa product improves the cardiometabolic profile in healthy and moderately hypercholesterolaemic adults. Br J Nutr. 2014;111(1):122-34.

127. West SG, McIntyre MD, Piotrowski MJ, Poupin N, Miller DL, Preston AG, et al. Effects of dark chocolate and cocoa consumption on endothelial function and arterial stiffness in overweight adults. Br J Nutr. 2014;111(4):653-61.

128. McFarlin BK, Venable AS, Henning AL, Prado EA, Best Sampson JN, Vingren JL, et al. Natural cocoa consumption: Potential to reduce atherogenic factors? J Nutr Biochem. 2015;26(6):626-32.

129. Balzer J, Rassaf T, Heiss C, Kleinbongard P, Lauer T, Merx M, et al. Sustained benefits in vascular function through flavanol-containing cocoa in medicated diabetic patients a double-masked, randomized, controlled trial. J Am Coll Cardiol. 2008;51(22):2141-9.

130. Neufingerl N, Zebregs YE, Schuring EA, Trautwein EA. Effect of cocoa and theobromine consumption on serum HDL-cholesterol concentrations: a randomized controlled trial. Am J Clin Nutr. 2013;97(6):1201-9.

131. Gonzales GF, Gonzales C, Gonzales-Castaneda C. Lepidium meyenii (Maca): a plant from the highlands of Peru-from tradition to science. Forsch Komplementärmed. 2009;16(6):373-80.

132. Stojanovska L, Law C, Lai B, Chung T, Nelson K, Day S, et al. Maca reduces blood pressure and depression, in a pilot study in postmenopausal women. Climacteric. 2015;18(1):69-78. 
133. Buono S, Langellotti AL, Martello A, Rinna F, Fogliano V. Functional ingredients from microalgae. Food Funct. 2014;5(8):1669-85.

134. Miczke A, Szulinska M, Hansdorfer-Korzon R, Kregielska-Narozna M, Suliburska J, Walkowiak J, et al. Effects of spirulina consumption on body weight, blood pressure, and endothelial function in overweight hypertensive Caucasians: a double-blind, placebo-controlled, randomized trial. Eur Rev Med Pharmacol Sci. 2016;20(1):150-6.

135. Ngo-Matip ME, Pieme CA, Azabji-Kenfack M, Biapa PC, Germaine N, Heike E, et al. Effects of Spirulina platensis supplementation on lipid profile in HIV-infected antiretroviral naive patients in Yaounde-Cameroon: a randomized trial study. Lipids Health Dis. 2014;13:191

136. Ngo-Matip ME, Pieme CA, Azabji-Kenfack M, Moukette BM, Korosky E, Stefanini P, et al. Impact of daily supplementation of Spirulina platensis on the immune system of naive HIV-1 patients in Cameroon: a 12-months single blind, randomized, multicenter trial. Nutr J. 2015;14:70.

137. Samuels R, Mani UV, Iyer UM, Nayak US. Hypocholesterolemic effect of spirulina in patients with hyperlipidemic nephrotic syndrome. J Med Food. 2002;5(2):91-6.

138. Lee EH, Park JE, Choi YJ, Huh KB, Kim WY. A randomized study to establish the effects of spirulina in type 2 diabetes mellitus patients. Nutr Res Pract. 2008;2(4):295-300.

139. Parikh $P$, Mani $U$, lyer $U$. Role of spirulina in the control of glycemia and lipidemia in Type 2 Diabetes Mellitus. J Med Food. 2001;4(4):193-9.

140. Ramamoorthy A, Premakumari S. Effect of supplementation of Spirulina on hypercholesterolemic patients. J Food Sci Technol. 1996;33(2):124-8.

141. Park HJ, Lee YJ, Ryu HK, Kim MH, Chung HW, Kim WY. A randomized double-blind, placebocontrolled study to establish the effects of spirulina in elderly Koreans. Ann Nutr Metab. 2008;52(4):322-8.

142. Rajoria A, Mehta A, Mehta P, Ahirwal L, Shukla S. Phytochemical analysis and estimation of major bioactive compounds from Triticum aestivum L. grass with antimicrobial potential. Pak J Pharm Sci. 2015.

143. Kumar N, lyer U. Impact of wheatgrass (Triticum aestivum L.) supplementation on atherogenic lipoproteins and menopausal symptoms in hyperlipidemic South Asian women - a randomized controlled study. J Diet Suppl. 2017;14(5):503-13.

144. Dutra RC, Campos MM, Santos AR, Calixto JB. Medicinal plants in Brazil: pharmacological studies, drug discovery, challenges and perspectives. Pharmacol Res. 2016;112:4-29.

145. Rocha AP, Carvalho LC, Sousa MA, Madeira SV, Sousa PJ, Tano T, et al. Endothelium-dependent vasodilator effect of Euterpe oleracea Mart. (Acai) extracts in mesenteric vascular bed of the rat. Vas Pharmacol. 2007;46(2):97-104.

146. Callaway J. Hempseed as a nutritional resource: an overview. Euphytica. 2004;140(1):65-72.

147. Mihoc M, Pop G, Alexa E, Radulov I. Nutritive quality of romanian hemp varieties (Cannabis sativa L.) with special focus on oil and metal contents of seeds. Chem Cent J. 2012;6(1):122.

148. Denisow B, Denisow-Pietrzyk M. Biological and therapeutic properties of bee pollen: a review. J Sci Food Agric. 2016;96(13):4303-9. 
149. Crozier SJ, Preston AG, Hurst JW, Payne MJ, Mann J, Hainly L, et al. Cacao seeds are a "Super Fruit": A comparative analysis of various fruit powders and products. Chem Cent J. 2011;5:5-.

150. Clements WT, Lee SR, Bloomer RJ. Nitrate ingestion: a review of the health and physical performance effects. Nutrients. 2014;6(11):5224-64.

151. Oude Groeniger J, van Lenthe FJ, Beenackers MA, Kamphuis CB. Does social distinction contribute to socioeconomic inequalities in diet: the case of 'superfoods' consumption. Int J Behav Nutr Phys Act. 2017;14(1):40.

152. Fraga CG, Litterio MC, Prince PD, Calabro V, Piotrkowski B, Galleano M. Cocoa flavanols: effects on vascular nitric oxide and blood pressure. J Clin Biochem Nutr. 2011;48(1):63-7.

153. Lila MA. Anthocyanins and human health: an in vitro investigative approach. J Biomed Biotechnol. 2004;2004(5):306-13.

154. De Pascual-Teresa S, Moreno DA, Garcia-Viguera C. Flavanols and anthocyanins in cardiovascular health: a review of current evidence. Int J Mol Sci. 2010;11(4):1679-703.

155. Li Y, Tran VH, Duke CC, Roufogalis BD. Preventive and protective properties of Zingiber officinale (ginger) in diabetes mellitus, diabetic complications, and associated lipid and other metabolic disorders: a brief review. J Evidence-Based Complementary Altern Med. 2012;2012:516870.

156. Li Y, Tran VH, Duke CC, Roufogalis BD. Gingerols of Zingiber officinale enhance glucose uptake by increasing cell surface GLUT4 in cultured L6 myotubes. Planta Med. 2012;78(14):1549-55.

157. Sekiya K, Ohtani A, Kusano S. Enhancement of insulin sensitivity in adipocytes by ginger. BioFactors. 2004;22(1-4):153-6.

158. Pérez-Torres I, Torres-Narváez JC, Pedraza-Chaverri J, Rubio-Ruiz ME, Díaz-Díaz E, del ValleMondragón L, et al. Effect of the aged garlic extract on cardiovascular function in metabolic syndrome rats. Molecules. 2016;21(11):1425.

159. Večeřa R, Orolin J, Škottová N, Kazdová L, Oliyarnik O, Ulrichová J, et al. The influence of maca (Lepidium meyenii) on antioxidant status, lipid and glucose metabolism in rat. Plant Foods Hum Nutri. 2007;62(2):59-63.

160. Deng R, Chow TJ. Hypolipidemic, antioxidant, and antiinflammatory activities of microalgae Spirulina. Cardiovasc Ther. 2010;28(4):e33-e45.

161. Kothari S, Jain AK, Mehta SC, Tonpay SD. Hypolipidemic effect of fresh Triticum aestivum (wheat) grass juice in hypercholesterolemic rats. Acta Pol Pharm. 2011;68(2):291-4.

162. Sethi J, Yadav M, Dahiya K, Sood S, Singh V, Bhattacharya S. Antioxidant effect of Triticum aestivium (wheat grass) in high-fat diet-induced oxidative stress in rabbits. Methods Find Exp Clin Pharmacol. 2010;32(4):233-5.

163. Yeh YY, Liu L. Cholesterol-lowering effect of garlic extracts and organosulfur compounds: human and animal studies. J Nutr. 2001;131(3s):989s-93s.

164. Nagaoka S, Shimizu K, Kaneko H, Shibayama F, Morikawa K, Kanamaru Y, et al. A novel protein C-phycocyanin plays a crucial role in the hypocholesterolemic action of Spirulina platensis concentrate in rats. J Nutr. 2005;135(10):2425-30.

165. Welty FK. How do elevated triglycerides and low HDL-cholesterol affect inflammation and atherothrombosis? Curr Cardiol Rep. 2013;15(9):400. 
166. Crouse JR, Grundy SM. Effects of alcohol on plasma lipoproteins and cholesterol and triglyceride metabolism in man. J Lipid Res. 1984;25(5):486-96.

167. Brinton EA. Effects of ethanol intake on lipoproteins. Curr Atheroscler Rep. 2012;14(2):108-14.

168. Eslick GD, Howe PR, Smith C, Priest R, Bensoussan A. Benefits of fish oil supplementation in hyperlipidemia: a systematic review and meta-analysis. Int J Cardiol. 2009;136(1):4-16.

169. Jafarnejad S, Keshavarz SA, Mahbubi S, Saremi S, Arab A, Abbasi S, et al. Effect of ginger (Zingiber officinale) on blood glucose and lipid concentrations in diabetic and hyperlipidemic subjects: A meta-analysis of randomized controlled trials. J Funct Foods. 2017;29:127-34.

170. Mazidi M, Gao H-K, Rezaie P, Ferns GA. The effect of ginger supplementation on serum C-reactive protein, lipid profile and glycaemia: a systematic review and meta-analysis. Food Nutr Res. 2016;60:32613-.

171. Serban MC, Sahebkar A, Dragan S, Stoichescu-Hogea G, Ursoniu S, Andrica F, et al. A systematic review and meta-analysis of the impact of Spirulina supplementation on plasma lipid concentrations. Clin Nutr. 2016;35(4):842-51.

172. Zhu Y, Miao Y, Meng Z, Zhong Y. Effects of Vaccinium berries on serum lipids: a meta-analysis of randomized controlled trials. J Evidence-Based Complementary Altern Med. 2015;2015:790329.

173. Zhu Y, Sun J, Lu W, Wang X, Wang X, Han Z, et al. Effects of blueberry supplementation on blood pressure: a systematic review and meta-analysis of randomized clinical trials. J Hum Hypertens. 2017;31(3):165-71.

174. Guo XF, Li ZH, Cai H, Li D. The effects of Lycium barbarum L. (L. barbarum) on cardiometabolic risk factors: a meta-analysis of randomized controlled trials. Food Funct. 2017;8(5):1741-8.

175. Ried K. Garlic lowers blood pressure in hypertensive individuals, regulates serum cholesterol, and stimulates immunity: an updated meta-analysis and review. J Nutr. 2016;146(2):389s-96s.

176. Zeng T, Guo FF, Zhang CL, Song FY, Zhao XL, Xie KQ. A meta-analysis of randomized, doubleblind, placebo-controlled trials for the effects of garlic on serum lipid profiles. J Sci Food Agric. 2012;92(9):1892-902.

177. Ried K, Toben C, Fakler P. Effect of garlic on serum lipids: an updated meta-analysis. Nutr Rev. 2013;71(5):282-99.

178. Hou LQ, Liu YH, Zhang YY. Garlic intake lowers fasting blood glucose: meta-analysis of randomized controlled trials. Asia Pac J Clin Nutr. 2015;24(4):575-82.

179. Mohammadi-Sartang M, Mazloom Z, Raeisi-Dehkordi H, Barati-Boldaji R, Bellissimo N, Totosy de Zepetnek JO. The effect of flaxseed supplementation on body weight and body composition: a systematic review and meta-analysis of 45 randomized placebo-controlled trials. Obes Rev. 2017;18(9):1096-107.

180. Khalesi S, Irwin C, Schubert M. Flaxseed consumption may reduce blood pressure: a systematic review and meta-analysis of controlled trials. J Nutr. 2015;145(4):758-65.

181. Ursoniu S, Sahebkar A, Andrica F, Serban C, Banach M. Effects of flaxseed supplements on blood pressure: A systematic review and meta-analysis of controlled clinical trial. Clin Nutr. 2016;35(3):615-25. 


\section{CHAPTER 2}

182. Pan A, Yu D, Demark-Wahnefried W, Franco OH, Lin X. Meta-analysis of the effects of flaxseed interventions on blood lipids. Am J Clin Nutr. 2009;90(2):288-97.

183. Desch S, Schmidt J, Kobler D, Sonnabend M, Eitel I, Sareban M, et al. Effect of cocoa products on blood pressure: systematic review and meta-analysis. Am J Hypertens. 2010;23(1):97-103.

184. Hooper L, Kay C, Abdelhamid A, Kroon PA, Cohn JS, Rimm EB, et al. Effects of chocolate, cocoa, and flavan-3-ols on cardiovascular health: a systematic review and meta-analysis of randomized trials. Am J Clin Nutr. 2012;95(3):740-51.

185. Jia L, Liu X, Bai YY, Li SH, Sun K, He C, et al. Short-term effect of cocoa product consumption on lipid profile: a meta-analysis of randomized controlled trials. Am J Clin Nutr. 2010;92(1):218-25.

186. Ried K, Fakler P, Stocks NP. Effect of cocoa on blood pressure. The Cochrane database of systematic reviews. 2017;4:Cd008893.

187. Tokede OA, Gaziano JM, Djousse L. Effects of cocoa products/dark chocolate on serum lipids: a meta-analysis. Eur J Clin Nutr. 2011;65(8):879-86.

188. Sansone R, Ottaviani JI, Rodriguez-Mateos A, Heinen Y, Noske D, Spencer JP, et al. Methylxanthines enhance the effects of cocoa flavanols on cardiovascular function: randomized, double-masked controlled studies. Am J Clin Nutr. 2017;105(2):352-60. 


\section{Supplemental materials}

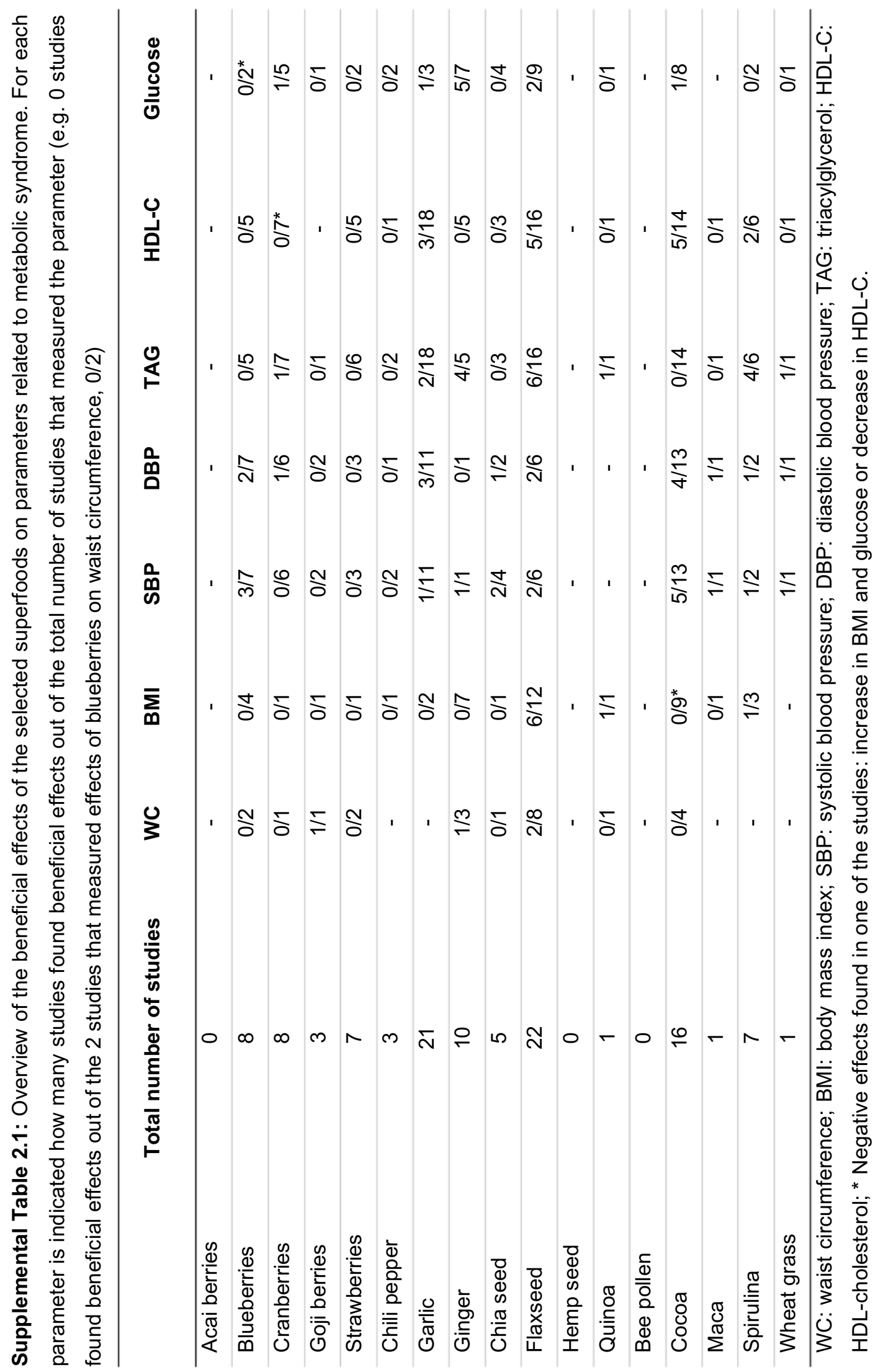





\section{CHAPTER 3}

\section{A single dose of goji berries does not affect postprandial energy expenditure and substrate oxidation in healthy, overweight men}

José J. van den Driessche, Jogchum Plat, Guy Plasqui, Ronald P. Mensink

Journal of Nutrition and Metabolism 2019 


\section{Abstract}

Background and aim: Increasing energy expenditure is an effective strategy for the prevention of obesity. In this respect, Lycium barbarum (goji berry) is of interest, as it has been shown to increase postprandial oxygen consumption. Although this suggests that energy expenditure was also increased, energy expenditure and substrate oxidation can only be assessed accurately when both oxygen consumption and carbon dioxide production are measured. We therefore investigated the effects of a single dose of Lycium barbarum fruit on postprandial energy expenditure and substrate oxidation in a randomized, double blind crossover trial. In addition, markers of lipid and glucose metabolism were measured.

Methods: Seventeen healthy, overweight men received in a random order a meal containing 25 grams of dried Lycium barbarum fruit or a control meal matched for caloric content and macronutrient composition. Energy expenditure and the respiratory quotient were determined using indirect calorimetry before and up to 4 hours after meal intake. Blood was sampled before and after meal intake at regular intervals for analyses of plasma glucose, serum triacylglycerol and free fatty acid concentrations.

Results: Energy expenditure significantly increased after the Lycium barbarum and control meal, but no differences were found between the meals $(p=0.217)$. Postprandial changes in respiratory quotient $(p=0.719)$ and concentrations of glucose $(p=0.663)$, triacylglycerol $(p=0.391)$ and free fatty acids $(p=0.287)$ were also not affected by Lycium barbarum intake.

Conclusions: A single dose of Lycium barbarum does not affect postprandial energy expenditure, substrate oxidation, and markers for lipid and glucose metabolism in healthy, overweight men. The trial was registered at clinicaltrials.gov as NCT02779985. 


\section{Introduction}

One of the main risk factors for the onset of cardiovascular diseases (CVDs) and type II diabetes mellitus is obesity, which develops when energy intake exceeds energy expenditure. ${ }^{1}$ Thus, increasing energy expenditure is a promising strategy to prevent obesity thereby lowering the risk to develop CVDs and type II diabetes mellitus. In addition, impaired fasting and postprandial fat oxidation have been linked to an increased risk for weight gain and obesity. ${ }^{2,3}$ In this light, foods affecting energy expenditure and fat oxidation are of interest.

Berries from the plant Lycium barbarum (goji berry, wolfberry) originate from Asia and have recently gained popularity in European countries. ${ }^{4}$ Major constituents of goji berries include polysaccharides, specifically referred to as Lycium barbarum polysaccharides (LBP), carotenoids (mainly zeaxanthin), and vitamins. ${ }^{5}$ Lycium barbarum has been consumed in Asia as part of traditional medicine for decades due to its potential beneficial effects on among others the development of CVDs and type II diabetes mellitus. It has also been suggested that the goji berry stimulates metabolism. Indeed, animal studies have indicated that expression of genes related to energy metabolism, such as UCP-1 and PGC1 $\alpha$, was elevated after Lycium barbarum intake. ${ }^{6}$ Unfortunately, energy expenditure was not measured in these experiments. However, the effect of Lycium barbarum on energy metabolism has been investigated in one human trial. In that study, it was found that a single dose of Lycium barbarum increased postprandial oxygen consumption in healthy men and women. ${ }^{7}$ Although this suggests that energy expenditure was increased, energy expenditure and substrate oxidation can only be assessed accurately when both oxygen consumption and carbon dioxide production are measured. The main objective of the current study was therefore to investigate the effects of a single dose of 25 grams dried Lycium barbarum fruit as part of a meal on postprandial energy expenditure and substrate oxidation in healthy, overweight men. In addition, effects on markers for postprandial lipid and glucose metabolism were studied, since postprandial hyperglycemia and hypertriglyceridemia are established risk factors for CVD. ${ }^{8,9}$ Overweight subjects were studied, as they are at increased risk to develop 
obesity and subsequently CVDs and type II diabetes mellitus, and might therefore benefit most from interventions targeting energy expenditure.

\section{Subjects and methods}

\section{Study population}

Apparently healthy overweight men were recruited using advertisement in local newspapers, online advertisements, and posters in university buildings and the hospital. Furthermore, subjects that had already participated in earlier studies from our department were approached. Men were invited for a screening visit if they met the following criteria: aged between 18 and 65 years, BMI between 25 and $30 \mathrm{~kg} / \mathrm{m}^{2}$, non-smoking, no use of anticoagulants or medications known to affect lipid or glucose metabolism, no conditions that might interfere with study outcomes, stable body weight ( $\leq 3 \mathrm{~kg}$ weight loss or gain in the past 3 months), no participation in another biomedical study during the past month and no abuse of drugs or alcohol. During a screening visit, fasting blood samples were taken for analysis of serum lipids and plasma glucose. In addition, height and weight were measured. Eighteen subjects were enrolled. These subjects had fasting serum triacylglycerol concentrations below $2.2 \mathrm{mmol} / \mathrm{L}$ and no elevated fasting serum cholesterol $(<8.0$ $\mathrm{mmol} / \mathrm{L})$ or plasma glucose $(<7.0 \mathrm{mmol} / \mathrm{L})$ concentrations. All subjects signed informed consent before the screening visit. This study was approved by the medical ethical committee of Maastricht University Medical Centre+ (MUMC+) and registered at clinicaltrials.gov as NCT02779985.

\section{Study design}

A randomized, double-blind, cross-over study with two treatments was carried out. For this, subjects visited the university during two occasions separated by a washout period of at least 7 days. On the day preceding each test day, subjects were asked to abstain from alcohol consumption, exercise and caffeine consumption (from 12.00 PM onwards) and to consume a standardized meal in the evening. Subjects were instructed to select a ready-made meal with a fixed macronutrient composition (30$40 \%$ fat, $40-50 \%$ carbohydrates and $13-16 \%$ proteins) from a list and to consume 
the same meal the evening before both test days to eliminate potential effects of the previous meal. ${ }^{10}$

After an overnight fast (from 08.00 PM), subjects came to the university by public transport or by car to limit physical activities in the morning as much as possible. Before the start of the measurements, subjects rested for 15 minutes in supine position and an intravenous catheter was placed for blood withdrawal. Next, indirect calorimetry was performed for 30 minutes and a blood sample was drawn (T0). Subjects were then asked to consume one of the two test meals in three equal parts divided over 10 minutes. After meal intake, indirect calorimetry measurements were continued for another 140 minutes. Next, the hood was removed and after a 20minute break during which subjects were allowed to stroll around, measurements were continued for another 40 minutes (T160 - T200). The last hour of the test days consisted of another 20-minute break and 40 minutes of indirect calorimetry measurements (T220 - T260). Blood samples were taken $15 \mathrm{~min}$ (T15), $30 \mathrm{~min}$ (T30), 45 min (T45), 60 min (T60), 90 min (T90), 120 min (T120), 180 min (T180) and $240 \mathrm{~min}$ (T240) after meal intake. A validated food frequency questionnaire was used to assess habitual food intake over the past month. Calorie content and macronutrient composition (Supplemental Table 3.1) was calculated using the Dutch food composition table (NEVO). Throughout the study, subjects were asked not to change their habitual diet and exercise pattern, and to record in a study diary their alcohol consumption and any changes in health status.

\section{Test meals}

At the two test days, subjects received a meal containing 25 grams of dried Lycium barbarum fruit ( $82 \mathrm{kcal}, 0.9 \mathrm{~g}$ fat, $13.3 \mathrm{~g}$ carbohydrates and $3.3 \mathrm{~g}$ protein per 25 grams; Superfood.nl, The Netherlands) or a control meal matched for macronutrient composition and energy content (Table 3.1). The amounts of carbohydrates, fat and proteins provided by Lycium barbarum fruit were in the control meal derived from plant-based sources. The Lycium barbarum and control meals had similar energy contents (684 kcal and $683 \mathrm{kcal}$ respectively) and macronutrient composition (55 En\% fat, 32 En\% carbohydrate, 12 En\% protein vs. 55 En\% fat, 33 En\% carbohydrate, $12 \mathrm{En} \%$ protein). Meals contained over 40 grams of fat to trigger a 
postprandial triacylglycerol response. ${ }^{11}$ The test meals, which were prepared by a research dietician, were flavored with caramel and presented in red, masked cups to blind the subject and the investigator.

Table 3.1: Macronutrient composition of the mixed test meals

\begin{tabular}{lcc}
\hline & $\begin{array}{c}\text { Lycium barbarum } \\
\text { meal * }\end{array}$ & $\begin{array}{c}\text { Control } \\
\text { meal }\end{array}$ \\
\hline Energy (kcal) & 684 & 683 \\
\hline Total Fat $(\mathrm{g})$ & 41.8 & 41.9 \\
$($ En\%) & 55 & 55 \\
\hline Carbohydrates $(\mathrm{g})$ & 54.4 & 56.0 \\
(En\%) & 32 & 33 \\
\hline Proteins $(\mathrm{g})$ & 20.3 & 20.3 \\
(En\%) & 12 & 12 \\
\hline
\end{tabular}

Values based on package information.

* Lycium barbarum meal contained $25 \mathrm{~g}$ dried Lycium barbarum, providing $82 \mathrm{kcal}, 0.9 \mathrm{~g}$ fat, $13.3 \mathrm{~g}$ carbohydrates, and $3.3 \mathrm{~g}$ protein.

\section{Indirect calorimetry}

Oxygen consumption $\left(\mathrm{VO}_{2}\right)$ and carbon dioxide production $\left(\mathrm{VCO}_{2}\right)$ were measured during fasting and postprandial conditions using a ventilated hood system (Omnical, Maastricht University, Maastricht, The Netherlands). Calibration of the indirect calorimeter was performed automatically every 30 min with span gas $\left(18 \% \mathrm{O}_{2}\right.$ and $\left.0.8 \% \mathrm{CO}_{2}\right)$ and nitrogen gas (100\%). Validation of the system was performed regularly during the study with a methanol combustion test. $\mathrm{VO}_{2}, \mathrm{VCO}_{2}$ and respiratory quotient (RQ) were averaged over 20 minutes at baseline (T0) and 10$30 \mathrm{~min}$ (T20), 30-50 min (T40), 50-70 min (T60), 70-90 min (T80), 90-110 $\mathrm{min}$ (T100), 110-130 $\mathrm{min}$ (T120), 170-190 $\mathrm{min}$ (T180) and 230-250 min (T240) after meal intake. Energy expenditure was calculated from $\mathrm{VO}_{2}$ and $\mathrm{VCO}_{2}$ data using the formula of Weir. ${ }^{12}$ Fat and carbohydrate oxidation were calculated using stoichiometric equations. ${ }^{13}$ 


\section{Biochemical analysis}

NaF-containing vacutainer tubes (Becton, Dickinson and Company, Franklin Lakes, $\mathrm{NJ}$, USA) were placed on ice immediately after blood withdrawal. Tubes were centrifuged within 30 minutes at $1300 \mathrm{xg}$ for 15 minutes at $4{ }^{\circ} \mathrm{C}$. Serum separator tubes (Becton, Dickinson and Company) were allowed to clot for 30-60 minutes at room temperature after withdrawal and centrifuged at $21{ }^{\circ} \mathrm{C}$ for 15 minutes at 1300 $x \mathrm{~g}$. All plasma and serum samples were directly frozen in liquid nitrogen and stored at $-80{ }^{\circ} \mathrm{C}$ until analysis.

At all time points, NaF plasma was used for analysis of glucose (Horiba ABX, Montpellier, France) and serum for analysis of free fatty acids (WAKO Chemicals $\mathrm{GmbH}$, Neuss, Germany). Serum triacylglycerol concentrations corrected for free glycerol (Sigma-Aldrich Corp., St. Louis, MO, USA) were measured at T0, T30, T60, T120, T180 and T240.

\section{Statistical analysis}

It was calculated that a sample size of 18 subjects was needed to detect a difference of $0.18 \mathrm{~kJ} / \mathrm{min}$ with a power of $80 \%$ and a within-subject variation of $0.25 \mathrm{~kJ} / \mathrm{min} .{ }^{14}$ All data is presented as mean $\pm S D$. Differences between test days in fasting values were compared using a paired samples T-test. Postprandial changes from baseline were analyzed using linear mixed models with meal and time as fixed factors and meal * time as interaction term. The interaction term was not significant in any of the models and therefore removed from all models. If the factor time was significant, time points were compared to baseline using post hoc tests with Bonferroni correction. The incremental area under the curve (iAUC), defined as the area above baseline values, was calculated with the trapezoidal rule ${ }^{15}$ for the 4 hours after meal intake. The decremental area under the curve (dAUC), defined as the area below baseline values, was calculated likewise. iAUCs and dAUCs were not normally distributed, as was apparent from Shapiro-Wilk tests. Therefore, values are presented as medians and ranges, and differences between test meals were compared using non-parametric tests. P-values $<0.05$ were considered statistically significant. Statistical analyses were performed with SPSS 21.0 for Mac (IBM Corp., Armonk, NY, USA). 


\section{Results}

\section{Subject characteristics}

Eighteen subjects were included and completed the study (Supplemental figure 3.1). One of the subjects was excluded from analysis due to clear absence of a postprandial response in all parameters measured during one of the test days. Average age of the remaining 17 subjects was $59.5 \pm 5.4$ years. Subjects were overweight with an average BMl of $27.2 \pm 1.4 \mathrm{~kg} / \mathrm{m}^{2}$. Inspection of the diaries did not reveal any protocol deviations that may have affected the results. Baseline characteristics of the 17 subjects who completed the study are presented in Table

\section{2 .}

Table 3.2: Baseline characteristics of the overweight men $(n=17)$

\begin{tabular}{lc}
\hline & Mean \pm SD \\
\hline Age $(\mathrm{y})$ & $59.5 \pm 5.4$ \\
\hline $\mathrm{BMI}\left(\mathrm{kg} / \mathrm{m}^{2}\right)$ & $27.2 \pm 1.4$ \\
\hline Weight $(\mathrm{kg})$ & $86.5 \pm 6.5$ \\
\hline Glucose $(\mathrm{mmol} / \mathrm{L})$ & $5.3 \pm 0.4$ \\
\hline Total cholesterol $(\mathrm{mmol} / \mathrm{L})$ & $5.3 \pm 0.7$ \\
\hline Triacylglycerol $(\mathrm{mmol} / \mathrm{L})$ & $1.2 \pm 0.4$ \\
\hline
\end{tabular}

\section{Energy expenditure}

Baseline energy expenditure did not differ between the two visits $(p=0.709$, data not shown). After meal intake, energy expenditure increased significantly $(p<0.01$ for factor time; Figure 3.1). No difference was found between the Lycium barbarum and control meals ( $p=0.217$ for factor meal). The iAUC over 4 hours was also not significantly different between the two meals $(p=0.113$, Supplemental table 3.2).

$\mathrm{VO}_{2}$ and $\mathrm{VCO}_{2}$ (Supplemental figure 3.2) did not differ between the Lycium barbarum and control meals at baseline $(p=0.673$ and $p=0.885$ respectively) and after meal intakes $(p=0.212$ and $p=0.431$ respectively). 


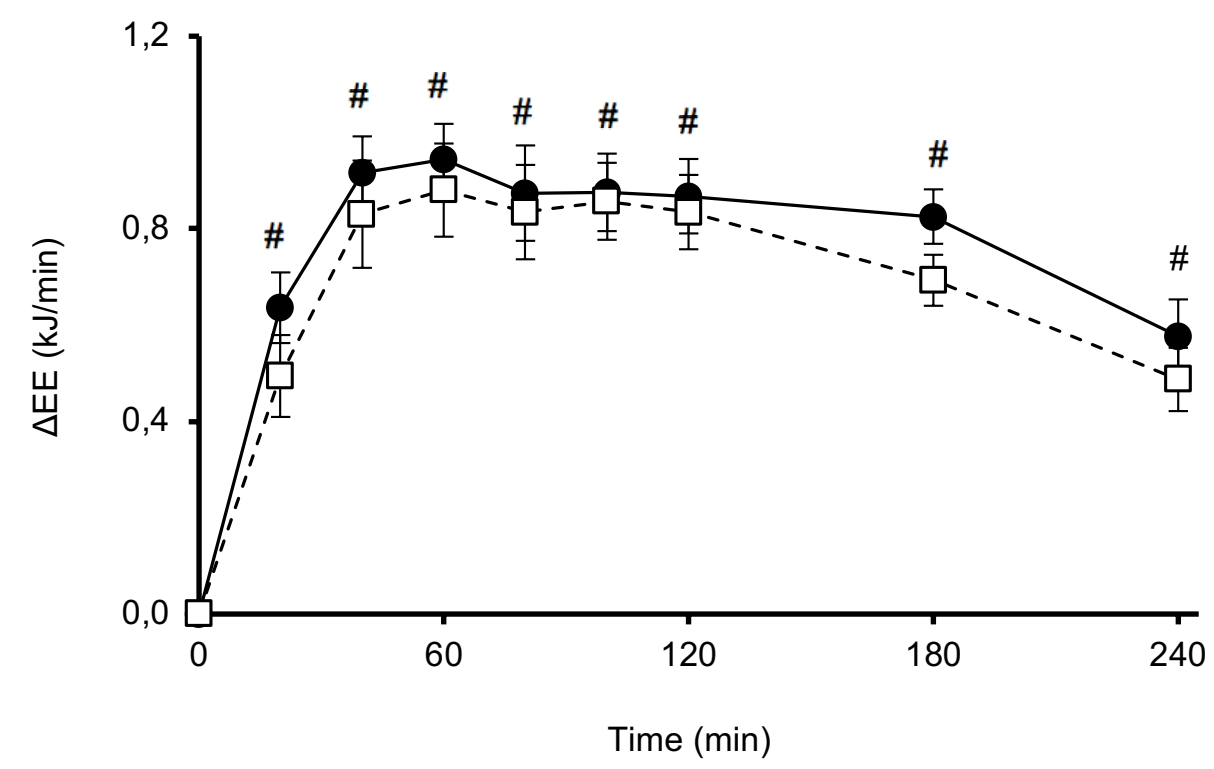

Figure 3.1: Mean changes $( \pm S E M)$ in energy expenditure $(E E)$ following the Lycium barbarum meal $(\square)$ and the control meal $(\bullet)$ in 17 healthy overweight men. Data was analyzed using linear mixed models. After Bonferroni correction, the factor time was significantly different from baseline values at all time points $(p<0.001)(\#)$.

\section{Respiratory quotient and substrate oxidation}

The $R Q$ at baseline did not differ between the test days ( $p=0.845$, data not shown), but increased 40 minutes after meal intake and returned to baseline after 240 minutes $(p<0.01$ for factor time; Figure 3.2). Changes over time were not affected by the intake of Lycium barbarum ( $p=0.719$ for factor meal and $p=0.523$ for iAUC,

\section{Supplemental table 3.2).}

Fat and carbohydrate oxidation (Supplemental figure 3.3) did not differ between the Lycium barbarum and control meals at baseline $(p=0.865$ and $p=0.991$, respectively) and after meal intake $(p=0.549$ and $p=0.909$ for factor meal respectively). 


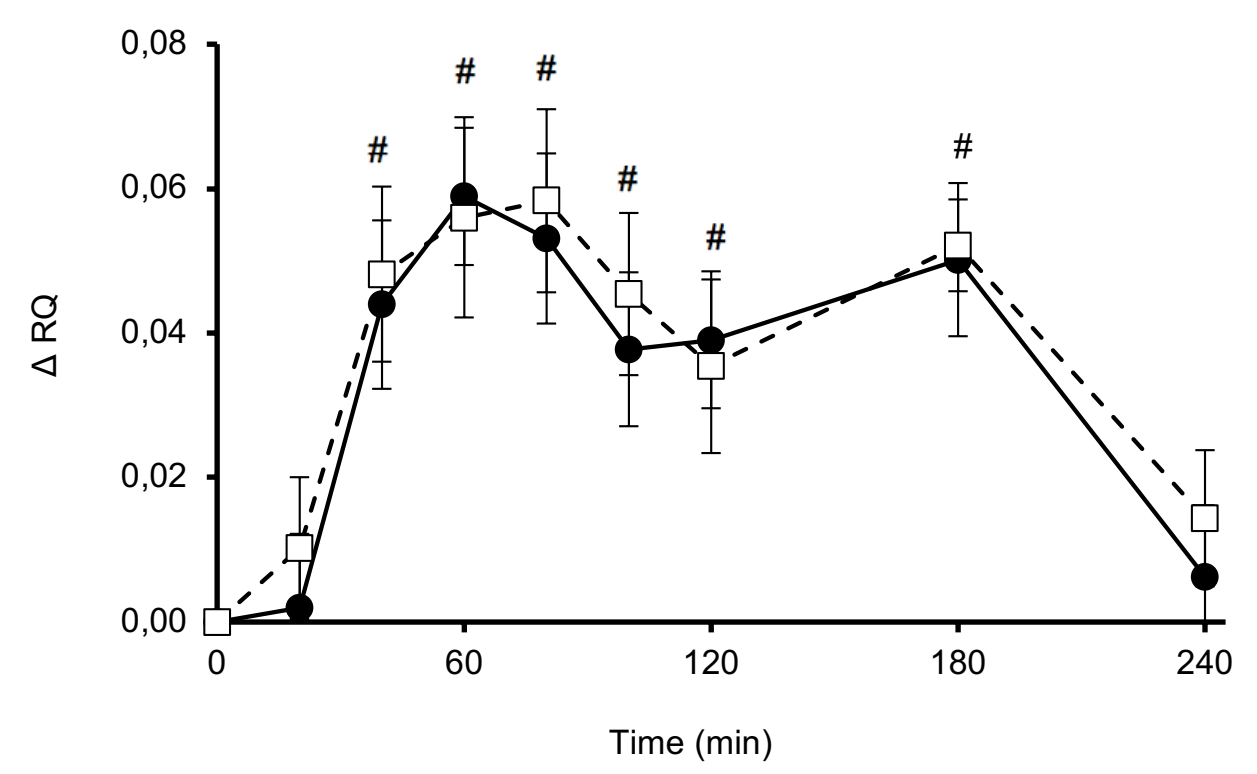

Figure 3.2: Mean changes $( \pm S E M)$ in respiratory quotient $(R Q)$ following the Lycium barbarum meal $(\square)$ and the control meal $(\bullet)$ in 17 healthy overweight men. Data was analyzed with linear mixed models. Significant effects compared to baseline $(P<0.001$ with Bonferroni correction) $(\#)$ were found for factor time.

\section{Glucose and lipid metabolism}

Plasma glucose concentrations at baseline did not differ between the two visits $(p=$ 0.724 , data not shown). Glucose concentrations increased significantly after meal intake and returned to baseline after 45 minutes $(p<0.01$ for factor time, Figure 3.3), but did not differ between meals $(p=0.663$ for factor meal and $p=0.332$ for iAUC, Supplemental table 3.2).

Serum triacylglycerol and FFA concentrations at baseline did not differ between test days ( $p=0.914$ and $p=0.330$ respectively). Sixty minutes after meal intake, triacylglycerol concentrations were increased and remained elevated throughout the test days ( $p<0.01$ for factor time, Figure 3.4). However, effects between the meals $(p=0.391$ for factor meal and $p=0.287$ for iAUC, Supplemental table 3.2) were not significantly different. FFA concentrations significantly decreased within 60 minutes after meal intake and returned to baseline concentrations after 240 minutes $(p<0.01$ for factor time, Figure 3.5). No differences were found between the meals $(p=0.133$ for factor meal and $p=0.723$ for $d A U C$, Supplemental table 3.2). 


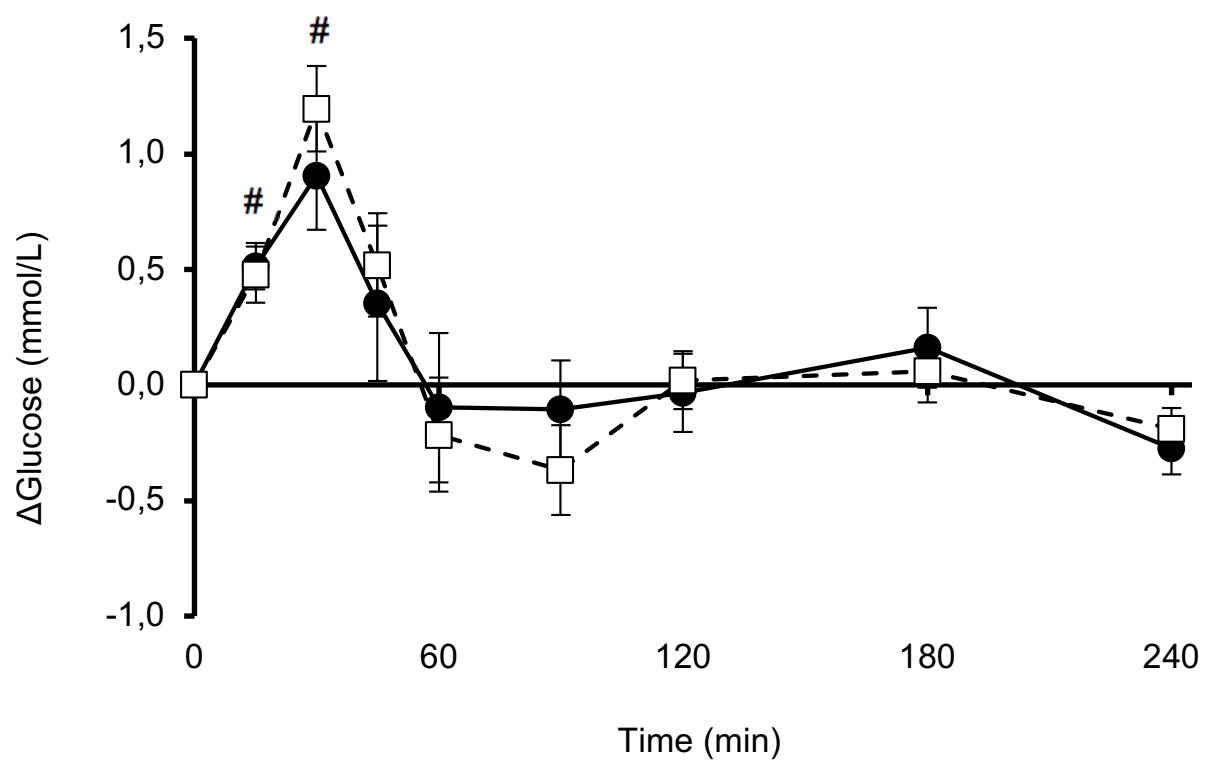

Figure 3.3: Mean changes ( \pm SEM) in plasma glucose concentrations following the Lycium barbarum meal $(\square)$ and the control meal $(\bullet)$ in 17 healthy overweight men. Data was analyzed using linear mixed models. Significant effects were found for factor time $(P<0.001$ with Bonferroni correction) (\#) compared to baseline.

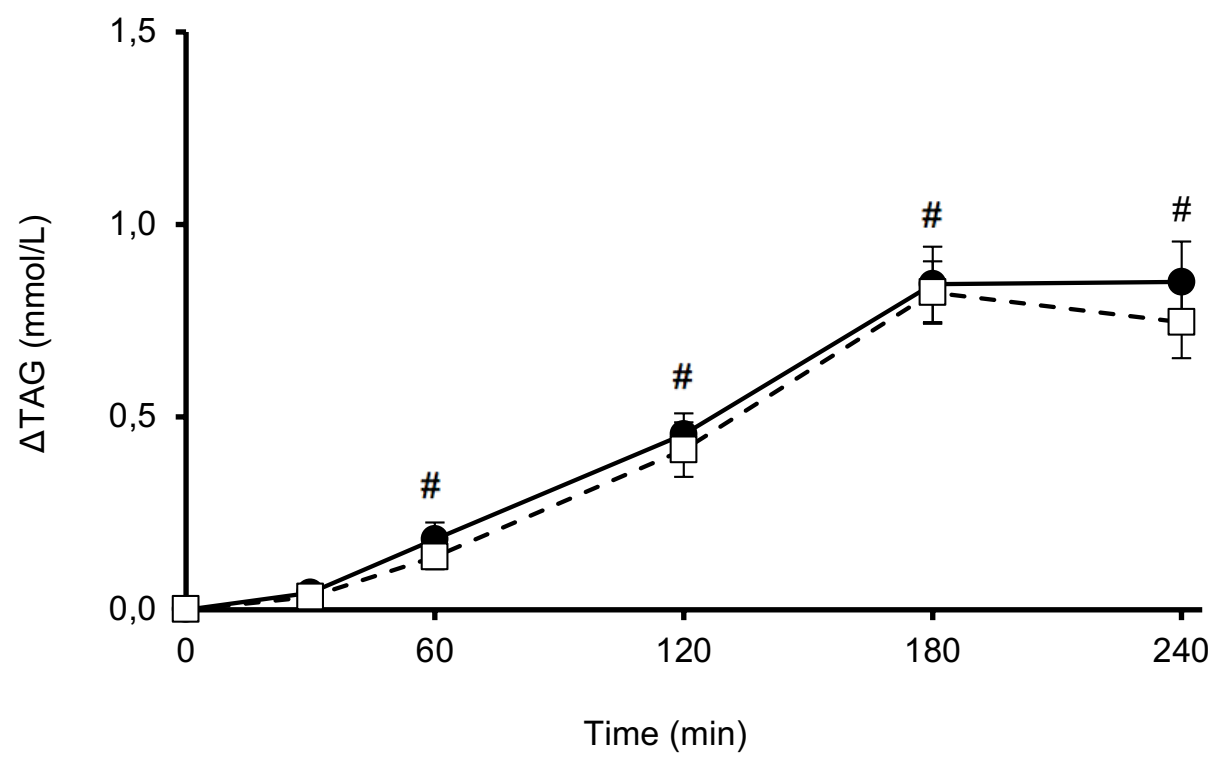

Figure 3.4: Mean changes ( \pm SEM) in serum triacylglycerol (TAG) concentrations following the Lycium barbarum meal $(\square)$ and the control meal $(\bullet)$ in 17 healthy overweight men. Data was analyzed using linear mixed models. Significant effects ( $p<0.05$, with Bonferroni correction) were found for factor time (\#) compared to baseline. 


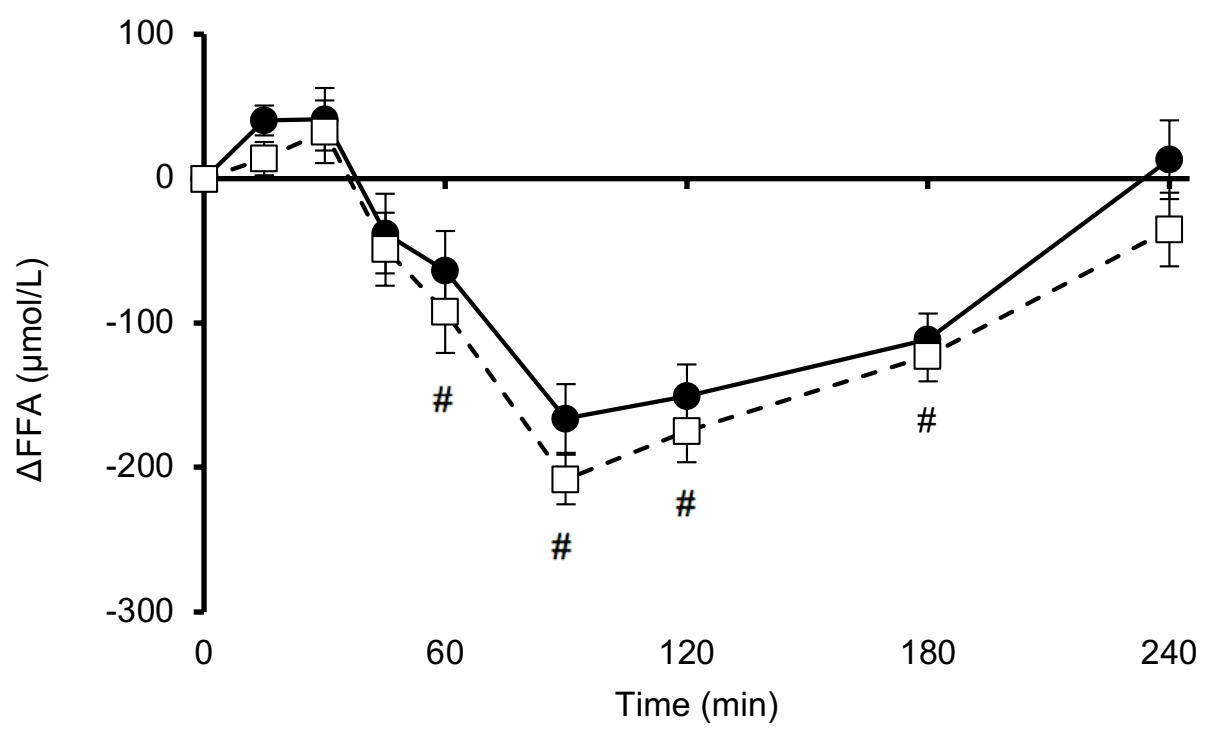

Figure 3.5: Mean changes ( \pm SEM) in serum free fatty acids (FFA) concentrations following the Lycium barbarum meal $(\square)$ and the control meal $(\bullet)$ in 17 healthy overweight men. Data was analyzed using linear mixed models. Significant effects $(P<0.001$, with Bonferroni correction) were found for factor time (\#) compared to baseline.

\section{Discussion}

In this study, we found no effects of a single dose of $25 \mathrm{~g}$ dried Lycium barbarum fruit on postprandial energy expenditure and substrate oxidation in healthy, overweight men. This contrasts findings in animal studies indicating that Lycium barbarum intake affected energy metabolism, as suggested by increased expression of genes involved in energy metabolism and increased brown adipose tissue activity. ${ }^{6}$ In two studies, ${ }^{6,16}$ body weight of mice and rats fed a high-fat diet also decreased after intake of Lycium barbarum extracts, whereas food intake was not altered. In these animal trials, water-soluble polysaccharides (LBP) were examined, which are thought to be the main bioactive component of goji berries. ${ }^{4}$ However, energy expenditure was not measured in these experiments. In one human study with 8 healthy overweight men and women, ${ }^{7}$ effects of single doses of Lycium barbarum on postprandial oxygen consumption were examined. Compared to placebo, only the highest dose of $120 \mathrm{ml}$ goji berry juice significantly increased oxygen consumption 1 and 4 hours after meal intake. One of the limitations of that trial, as also discussed by the authors, was the small sample size. Additionally, only oxygen 
consumption was measured. To assess energy expenditure and substrate oxidation accurately, both oxygen consumption and carbon dioxide production need to be measured, as we did. Nonetheless, as already mentioned, no effects were found, also not on oxygen consumption. We have no obvious explanation for these discrepancies in results. Differences in subject characteristics may have played a role. Although in both trials healthy overweight subjects participated, participants in the trial of Amagase and Nance were younger ( $34.5 \pm 7$ years), while both men and women were included.

The single dose of 25 grams of dried Lycium barbarum fruit used in the current study fits within the range of $15-30$ grams of the dried fruit frequently used in traditional Asian medicine. ${ }^{5}$ In the study of Amagase and Nance, $120 \mathrm{ml}$ of LBPstandardized goji berry juice was used, corresponding to the amount of LBP found in 150 grams of fresh berries. This dose is comparable to the amount of dried fruit used in our trial. Although the Lycium barbarum provided in both trials was processed differently, it is unlikely that this explains the difference in results. Different preparations of Lycium barbarum, such as powders or juices, ${ }^{17,18}$ and extracts isolated from the dried fruit have been found to be biologically active,${ }^{19}$ suggesting that potentially active constituents are not lost during drying.

In both trials, Lycium barbarum was provided with a meal, but caloric intake as well as macronutrient composition of the meals were different between the two studies. In the study of Amagase and Nance, the caloric content of the highest dose of goji berry juice combined with the meal was $260 \mathrm{kcal}$ less than our meal. The meal, excluding the goji berry juice, provided $55 \%$ of the energy from carbohydrates, whereas our meal provided $55 \%$ of the energy from fat. However, whether differences in meal composition can explain the discrepancies in results warrants further study.

No longer-term studies have been performed investigating the effects of Lycium barbarum intake on measures of energy expenditure. However, some studies have measured changes in body weight, which could serve as a proxy for energy balance. In mice, body weight was reduced after longer-term Lycium barbarum intake. ${ }^{6,20}$ In humans, however, a recent meta-analysis did not find any effects of Lycium 
barbarum intake on body weight. ${ }^{21}$ In the five trials included, a total of 366 subjects were supplemented with Lycium barbarum or placebo for 14 days to 3 months. ${ }^{17,18,22-}$ ${ }^{24}$ In none of these trials, food intake was controlled. Though suggestive, from this meta-analysis it cannot definitely be concluded that energy expenditure did not change, since other factors might have influenced body weight as well in these trials.

Another question is whether Lycium barbarum may reduce cardiovascular disease risk by improving postprandial lipid and glucose metabolism. Only one other study has addressed this question. This was, however, an intervention trial with intake of Lycium barbarum over three months instead of a single dose. Nevertheless, postprandial glucose concentrations were lowered in this trial. ${ }^{24}$ In rats, LBP administration for four weeks stimulated translocation and activation of glucose transporter isoform 4 (GLUT4) in adipocytes and lowered glucose concentrations. ${ }^{25}$ Furthermore, LBPs have been shown to reduce intestinal glucose uptake in Caco-2 cells. ${ }^{26}$ In our study, no changes in postprandial glucose concentrations were found. One may argue that a single dose of Lycium barbarum might not be sufficient to induce changes in glucose concentrations, whereas repeated intake does. In the study of Cai et al, ${ }^{24}$ no effects of repeated Lycium barbarum consumption were found on postprandial triacylglycerol concentrations, which is in line with the results from our single dose study.

In summary, our study indicates that a single dose of Lycium barbarum does not influence postprandial energy expenditure and substrate oxidation in healthy, overweight men. Furthermore, Lycium barbarum intake did not affect postprandial plasma glucose, serum free fatty acids and triacylglycerol concentrations.

\section{Acknowledgements}

The authors would like to thank Martine Hulsbosch, Maud Beckers and Cara op 't Eyndt for technical and dietary assistance throughout the study. 


\section{References}

1. Haslam DW, James WP. Obesity. Lancet (London, England). 2005;366(9492):1197-209.

2. Marra M, Scalfi L, Contaldo F, Pasanisi F. Fasting respiratory quotient as a predictor of long-term weight changes in non-obese women. Ann Nutr Metab. 2004;48(3):189-92.

3. Westerterp KR, Smeets A, Lejeune MP, Wouters-Adriaens MP, Westerterp-Plantenga MS. Dietary fat oxidation as a function of body fat. Am J Clin Nutr. 2008;87(1):132-5.

4. Potterat O. Goji (Lycium barbarum and L. chinense): Phytochemistry, pharmacology and safety in the perspective of traditional uses and recent popularity. Planta Med. 2010;76(1):7-19.

5. Amagase H, Farnsworth NR. A review of botanical characteristics, phytochemistry, clinical relevance in efficacy and safety of Lycium barbarum fruit (Goji). Food Res Int. 2011;44(7):1702-17.

6. Li W, Li Y, Wang Q, Yang Y. Crude extracts from Lycium barbarum suppress SREBP-1c expression and prevent diet-induced fatty liver through AMPK activation. BioMed Res Int. 2014;2014:196198.

7. Amagase $\mathrm{H}$, Nance DM. Lycium barbarum increases caloric expenditure and decreases waist circumference in healthy overweight men and women: pilot study. J Am Coll Nutr. 2011;30(5):3049.

8. Hanefeld M, Koehler C, Schaper F, Fuecker K, Henkel E, Temelkova-Kurktschiev T. Postprandial plasma glucose is an independent risk factor for increased carotid intima-media thickness in nondiabetic individuals. Atherosclerosis. 1999;144(1):229-35.

9. Ginsberg HN, Illingworth DR. Postprandial dyslipidemia: an atherogenic disorder common in patients with diabetes mellitus. Am J Cardiol. 2001;88(6a):9h-15h.

10. Robertson MD, Henderson RA, Vist GE, Rumsey RD. Extended effects of evening meal carbohydrate-to-fat ratio on fasting and postprandial substrate metabolism. Am J Clin Nutr. 2002;75(3):505-10.

11. Dubois C, Beaumier G, Juhel C, Armand M, Portugal H, Pauli AM, et al. Effects of graded amounts (0-50 g) of dietary fat on postprandial lipemia and lipoproteins in normolipidemic adults. Am J Clin Nutr. 1998;67(1):31-8.

12. Weir JB. New methods for calculating metabolic rate with special reference to protein metabolism. J Physiol. 1949;109(1-2):1-9.

13. Frayn KN. Calculation of substrate oxidation rates in vivo from gaseous exchange. J Appl Physiol: Respir Environ Exercise Physiol. 1983;55(2):628-34.

14. Faul F, Erdfelder E, Lang AG, Buchner A. G*Power 3: A flexible statistical power analysis program for the social, behavioral, and biomedical sciences. Behavior Research Methods. 2007;39, 175191.

15. Matthews JN, Altman DG, Campbell MJ, Royston P. Analysis of serial measurements in medical research. Br Med J. 1990;300(6719):230-5.

16. Xiao J, Xing F, Huo J, Fung ML, Liong EC, Ching YP, et al. Lycium barbarum polysaccharides therapeutically improve hepatic functions in non-alcoholic steatohepatitis rats and cellular steatosis model. Sci Rep. 2014;4:5587. 


\section{CHAPTER 3}

17. Amagase $H$, Sun B, Nance DM. Immunomodulatory effects of a standardized Lycium barbarum fruit juice in Chinese older healthy human subjects. J Med Food. 2009;12(5):1159-65.

18. Vidal K, Bucheli P, Gao Q, Moulin J, Shen LS, Wang J, et al. Immunomodulatory effects of dietary supplementation with a milk-based wolfberry formulation in healthy elderly: a randomized, doubleblind, placebo-controlled trial. Rejuvenation Res. 2012;15(1):89-97.

19. Zhu J, Liu W, Yu J, Zou S, Wang J, Yao W, et al. Characterization and hypoglycemic effect of a polysaccharide extracted from the fruit of Lycium barbarum L. Carbohydr Polym. 2013;98(1):8-16.

20. Zhang M, Wang J, Zhang S. Study on the composition of Lycium barbarum polysaccharides and its effects on the growth of weanling mice. Wei sheng yan jiu. 2002;31(2):118-9. (Only abstract available in English)

21. Guo XF, Li ZH, Cai H, Li D. The effects of Lycium barbarum L. (L. barbarum) on cardiometabolic risk factors: a meta-analysis of randomized controlled trials. Food Funct. 2017;8(5):1741-8.

22. Amagase H, Nance DM. A randomized, double-blind, placebo-controlled, clinical study of the general effects of a standardized Lycium barbarum (Goji) Juice, GoChi. J Altern Complement Med. 2008;14(4):403-12.

23. Amagase $\mathrm{H}$, Sun $\mathrm{B}$, Borek $\mathrm{C}$. Lycium barbarum (goji) juice improves in vivo antioxidant biomarkers in serum of healthy adults. Nutr Res. 2009;29(1):19-25.

24. Cai H, Liu F, Zuo P, Huang G, Song Z, Wang T, et al. Practical application of antidiabetic efficacy of Lycium barbarum polysaccharide in patients with type 2 diabetes. Med Chem. 2015;11(4):38390.

25. Zhao R, Qiu B, Li Q, Zhang T, Zhao H, Chen Z, et al. LBP-4a improves insulin resistance via translocation and activation of GLUT4 in OLETF rats. Food Funct. 2014;5(4):811-20.

26. Cai H, Yang X, Cai Q, Ren B, Qiu H, Yao Z. Lycium barbarum L. polysaccharide (LBP) reduces glucose uptake via down-regulation of SGLT-1 in Caco2 cell. Molecules. 2017;22(2). 


\section{Supplemental materials}

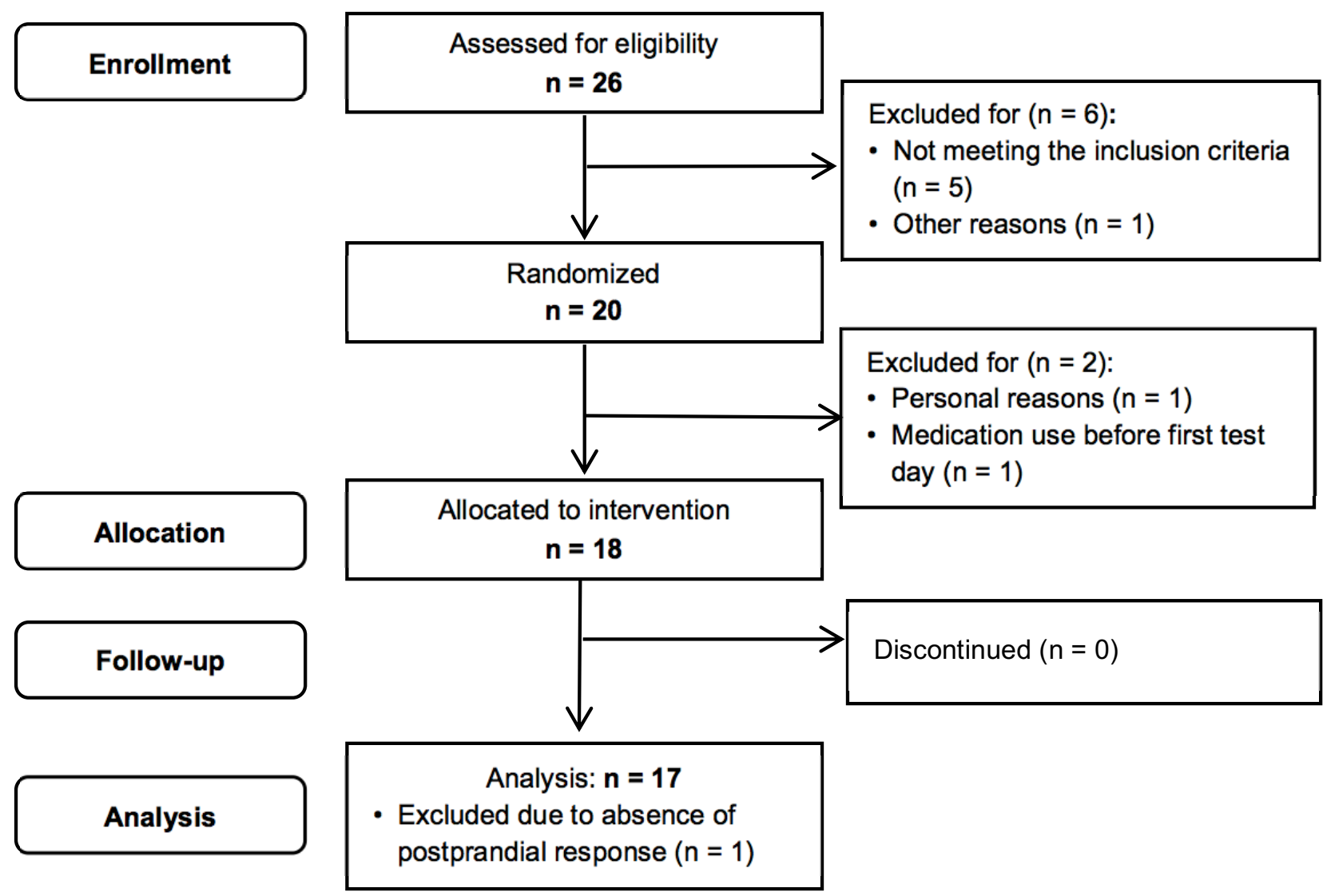

Supplemental figure 3.1: Flow chart of inclusion and exclusion of participants throughout this randomized, crossover study 


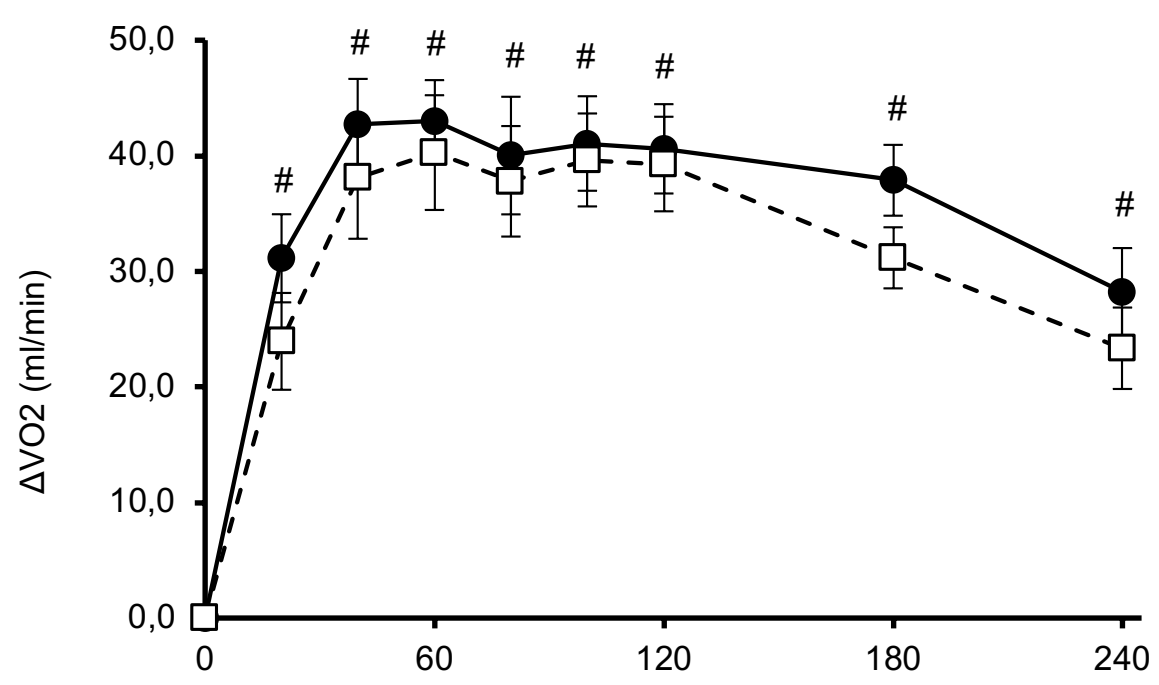

A

Time (min)

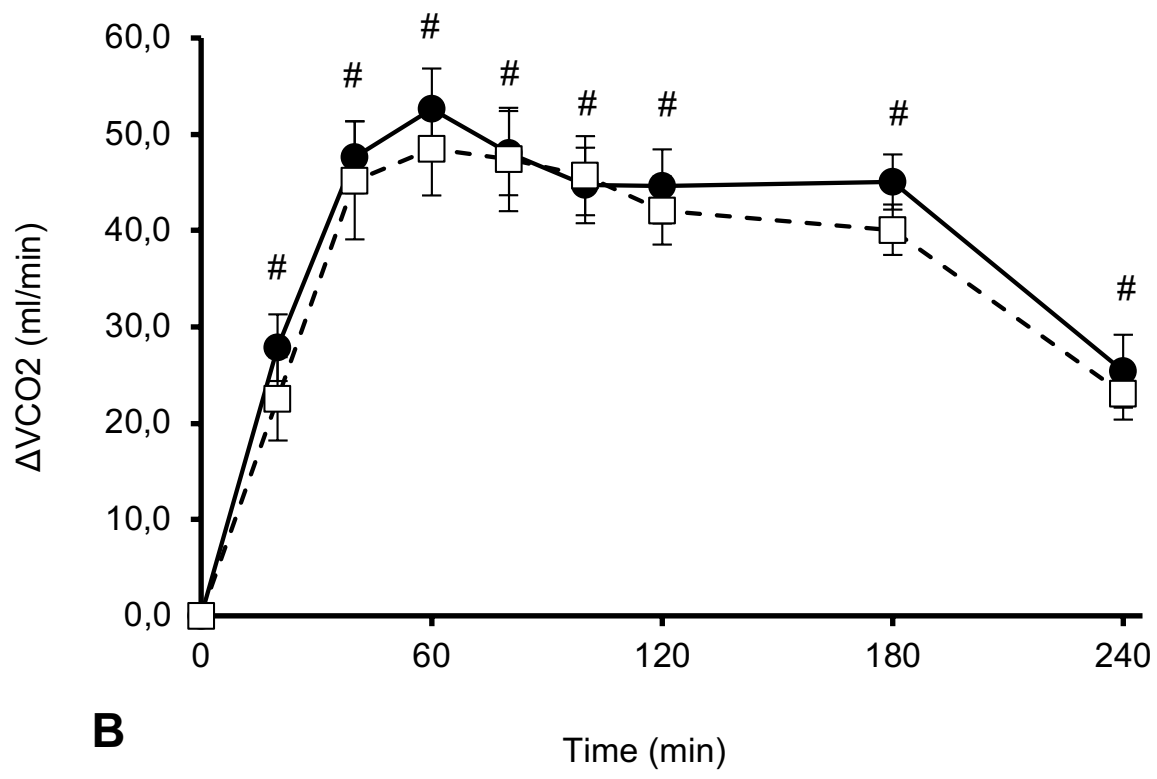

Supplemental figure 3.2: Mean changes ( $\pm \mathrm{SEM})$ in oxygen consumption $\left(\mathrm{VO}_{2}\right.$; panel $\left.\mathrm{A}\right)$ and carbon dioxide production ( $\mathrm{VCO}_{2}$; panel B) following the Lycium barbarum meal $(\square)$ and the control meal $(\bullet)$ in 17 healthy overweight men. Data was analyzed using linear mixed models. Significant effects $(P<0.001$, with Bonferroni correction) were found for factor time (\#) compared to baseline. 

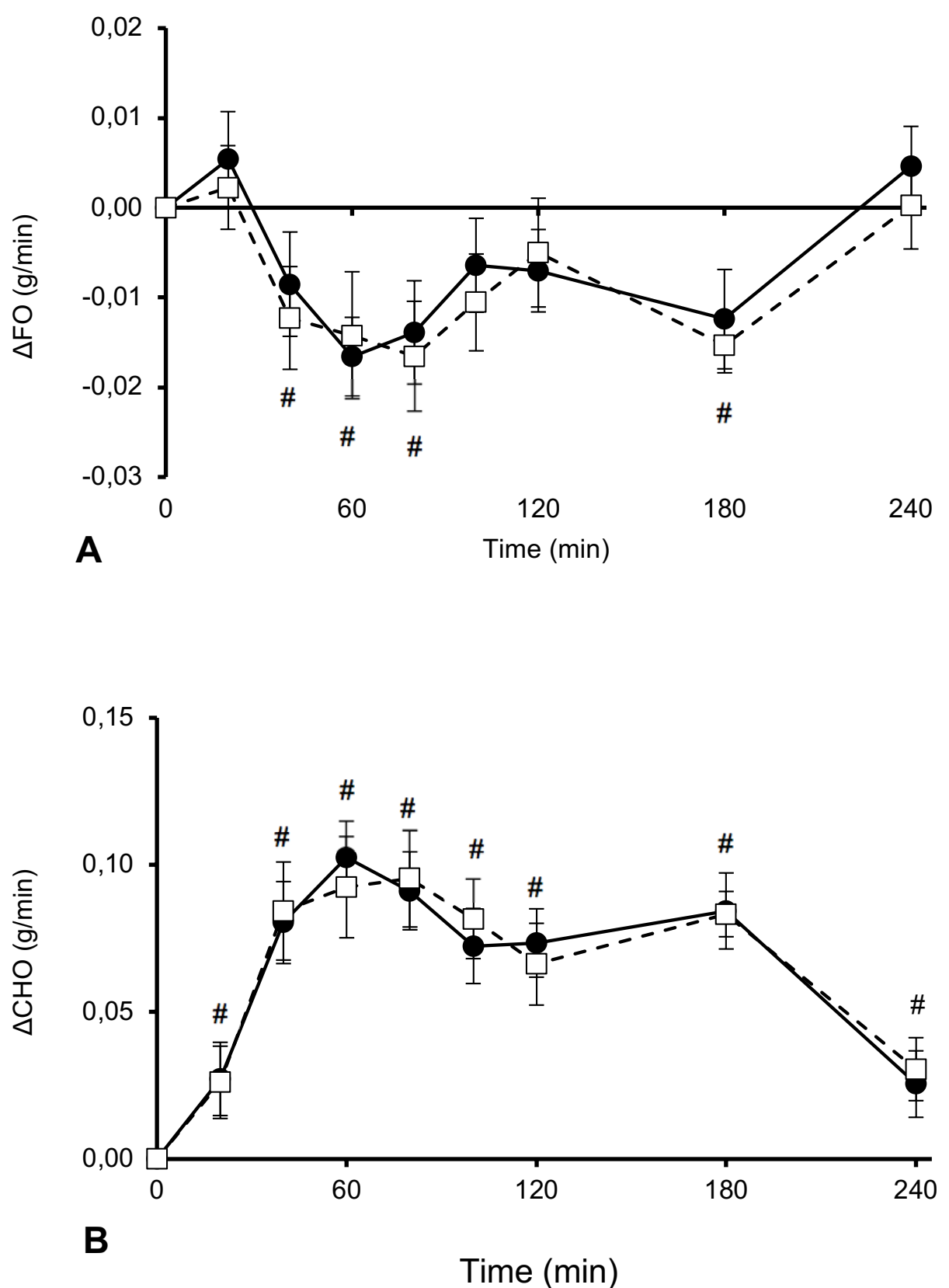

Supplemental figure 3.3: Mean changes $( \pm$ SEM) in fat oxidation (FO; panel A) and carbohydrate oxidation (CHO; panel B) following the Lycium barbarum meal $(\square)$ and the control meal $(\bullet)$ in 17 healthy overweight men. Data was analyzed using linear mixed models. Significant effects $(P<0.001$, with Bonferroni correction) were found for factor time (\#) compared to baseline. 
Supplemental table 3.1: habitual dietary intake of the men who participated in the trial as determined by a validated food frequency questionnaire.

\begin{tabular}{lc}
\hline & Medians with ranges \\
\hline Energy (kcal/day) & $2544(1773-4425)$ \\
\hline Fat (energy \%) & $35.6(26.7-41.1)$ \\
SFA & $12.5(8.7-14.0)$ \\
MUFA & $12.2(8.2-16.9)$ \\
PUFA & $7.3(4.9-10.4)$ \\
\hline Protein (energy \%) & $16.2(11.9-19.7)$ \\
\hline Carbohydrates (energy \%) & $43.8(28.1-50.1)$ \\
\hline Alcohol (energy \%) & $3.2(0.0-12.7)$ \\
\hline Fiber (g/day) & $31.4(20.1-72.3)$ \\
\hline Cholesterol (mg/day) & $270(123-435)$ \\
\hline
\end{tabular}

Supplemental table 3.2: iAUC/dAUC over 4 hours for energy expenditure $(E E)$, respiratory quotient $(R Q)$, and concentrations of plasma glucose, serum free fatty acids (FFA) and serum triacylglycerol (TAG) after Lycium barbarum and control meal intake ${ }^{1}$

\begin{tabular}{llccc}
\hline & & $\begin{array}{c}\text { Lycium barbarum } \\
\text { meal }\end{array}$ & $\begin{array}{c}\text { Control } \\
\text { meal }\end{array}$ \\
\hline $\mathrm{EE}$ & $\mathrm{kJ}$ per $240 \mathrm{~min}$ & iAUC & $167(90-269)$ & $176(126-298)$ \\
\hline $\mathrm{RQ}$ & per $240 \mathrm{~min}$ & iAUC & $10.6(0.0-18.9)$ & $8.9(1.5-21.8)$ \\
\hline Glucose & $\mathrm{mmol} / \mathrm{L}$ per $240 \mathrm{~min}$ & iAUC & $58.4(6.1-225.4)$ & $64.0(3.5-290.3)$ \\
\hline FFA & $\mathrm{mol}^{*} 10^{3} / \mathrm{L}$ per $240 \mathrm{~min}$ & dAUC & $26.9(0.9-57.8)$ & $16.8(3.2-43.9)$ \\
\hline TAG & $\mathrm{mmol} / \mathrm{L}$ per $240 \mathrm{~min}$ & iAUC & $108(25-202)$ & $101(29-251)$ \\
\hline
\end{tabular}

${ }^{1}$ Data is presented as medians with ranges. 


\section{CHAPTER 4}

Effects of spirulina and wakame consumption on intestinal cholesterol absorption and serum lipid concentrations in non-hypercholesterolemic adult men and women

José J. van den Driessche, Jogchum Plat, Maurice C.J.M. Konings, Ronald P. Mensink 


\begin{abstract}
Purpose: Consumption of the algae spirulina (Arthrospira platensis or maxima) and wakame (Undaria pinnatifida) has been shown to lower LDL cholesterol concentrations in animals and humans, possibly due to inhibition of intestinal cholesterol absorption. This mechanism, however, has never been investigated in humans. Therefore, we examined in non-hypercholesterolemic men and women effects of spirulina and wakame consumption on serum markers for intestinal cholesterol absorption.
\end{abstract}

Method: Thirty-five healthy men and women without hypercholesterolemia consumed in a random order daily 4.8 grams spirulina, wakame or placebo for 17 days, separated by 14-day washouts. After 17 days, serum cholesterol-standardized campesterol, sitosterol and cholestanol, and lathosterol concentrations were measured as markers for intestinal cholesterol absorption and cholesterol synthesis respectively. Concentrations of serum total cholesterol, LDL and HDL cholesterol, triacylglycerol, and plasma glucose, and blood pressure were measured as well.

Results: Compared with placebo, spirulina or wakame did not affect serum cholesterol-standardized campesterol $(\mathrm{Cl}:-0.23-0.10 \mu \mathrm{mol} / \mathrm{mmol}, \mathrm{P}=0.435$ and Cl: $-0.14-0.19 \mu \mathrm{mol} / \mathrm{mmol}, P=0.729$, respectively), sitosterol $(P=0.314$ and $P$ $=0.112)$, cholestanol $(P=0.610$ and $P=0.809)$, or lathosterol $(P=0.388$ and $P=$ $0.102)$ concentrations. In addition, serum lipid and plasma glucose concentrations, and blood pressure were not changed.

Conclusions: Daily consumption of 4.8 grams spirulina or wakame for 17 days did not affect plasma markers for intestinal cholesterol absorption or cholesterol synthesis in non-hypercholesterolemic men and women. Serum lipid and glucose concentrations, and blood pressure were also not altered. 


\section{Introduction}

Lowering serum LDL cholesterol (LDL-C) concentrations is a well-established strategy to reduce cardiovascular disease (CVD) risk. ${ }^{1}$ This can be realized via inhibiting intestinal cholesterol absorption or suppressing endogenous cholesterol synthesis. ${ }^{2}$ In this respect, not only drugs, but also diet plays an important role. Proven examples of natural compounds or foods affecting cholesterol absorption or synthesis include plant sterols and stanols, fibers, and red yeast rice. ${ }^{3,4}$ However, also other foods, such as algae, may contain bioactive components that lower serum LDL-C concentrations.

Consumption of algae has gained popularity in the Western world over the past few years, due to their postulated beneficial effects on CVD risk. ${ }^{5,6}$ Spirulina (Arthrospira platensis or maxima), belonging to the family of cyanobacteria, is a microalga containing high amounts of proteins, vitamins and light-harvesting structures such as C-phycocyanin. ${ }^{7}$ Animal $^{8,9}$ and several - but not all - human trials ${ }^{10-15}$ have suggested that spirulina lowers serum total cholesterol (TC) and / or LDL-C concentrations. Studies in rats have now suggested that inhibition of intestinal cholesterol absorption could be the mechanism underlying the LDL-C reduction. ${ }^{9}$ Wakame (Undaria pinnatifida) is one of the most-consumed macro algae worldwide. ${ }^{16}$ Constituents in wakame include the carotenoid fucoxanthin and fucoidan, a polysaccharide found in brown algae. ${ }^{17} \mathrm{~A}$ limited number of studies has evaluated the cholesterol-lowering effects of wakame. Results from studies in rats on the effects of wakame ${ }^{18-20}$ or its extract fucoxanthin ${ }^{21}$ showed reductions in serum TC or LDL-C concentrations. On the other hand, three human studies did not show cholesterol-lowering effects of wakame, ${ }^{22-24}$ whereas a trial using fucoidan extracts from wakame did. ${ }^{25}$ Again, inhibition of intestinal cholesterol absorption has been suggested as the underlying mechanism. ${ }^{19,21}$

Taken together, there is evidence both from human and animal studies that spirulina and wakame lower LDL-C concentrations, possibly by inhibition of intestinal cholesterol absorption. However, this mechanism has never been examined in humans. Therefore, the aim of the present study was to evaluate in healthy, nonhypercholesterolemic men and women effects of spirulina and wakame consumption 
on markers for intestinal cholesterol absorption and endogenous cholesterol synthesis, and on serum lipid concentrations. Effects on glucose concentrations and blood pressure, as additional CVD risk markers, ${ }^{26}$ were studied as well.

\section{Subjects and methods}

\section{Study population}

Thirty-six apparently healthy men and women were recruited via online advertisements, posters in university and hospital buildings, and among subjects who had already participated in earlier studies within our department. Subjects were eligible for participation if they met the following criteria: aged between 18 and 70 years, BMI between 18 and $30 \mathrm{~kg} / \mathrm{m}^{2}$, non-smoking, no use of medication or food supplements known to affect lipid or glucose metabolism or blood pressure, no conditions that might interfere with study outcomes, stable body weight $(\leq 3 \mathrm{~kg}$ weight loss or gain in the past 3 months), no participation in another biomedical trial during the past month, and no abuse of drugs or alcohol. During a screening visit, fasting blood samples were taken to exclude subjects with elevated serum TC $(\geq 8.0$ $\mathrm{mmol} / \mathrm{L})$, serum triacylglycerol ( $\geq 4.5 \mathrm{mmol} / \mathrm{L}$ ) or plasma glucose $(\geq 7.0 \mathrm{mmol} / \mathrm{L})$ concentrations. Furthermore, weight and height were measured for determination of BMI. All subjects signed informed consent before the screening visit. This study was approved by the medical ethical committee of Maastricht University Medical Centre+ (MUMC+) and registered at clinicaltrials.gov as NCT03380611.

\section{Study design and intervention products}

The study had a randomized, placebo-controlled, double-blind crossover design with three intervention periods of 17 days each, separated by washout periods of at least 14 days. Subjects were randomly assigned to one of the six possible treatment sequences for spirulina, wakame or placebo consumption. During the 17-days intervention periods, subjects consumed daily 12 capsules, each containing either $400 \mathrm{mg}$ spirulina (Flora Health, Burnaby, Canada), 400 mg wakame (Swanson Health, Fargo, North Dakota, USA), or 400 mg microcrystalline cellulose (Radboud UMC, Nijmegen, the Netherlands). Thus, in total 4.8 grams spirulina, 4.8 grams 
wakame or placebo had to be consumed daily. This dose was used since it approximates the average dosage used in former studies with spirulina and wakame. ${ }^{22-24,27}$ Sterol composition of the spirulina and wakame capsules was measured using gas-chromatography flame-ionization-detection (GC-FID) by Bonn University (Supplemental table 4.1). All capsules were different in appearance and subjects were not informed about the content of the capsules. At the start of each intervention period, capsules were provided in sachets labeled with $A, B$ or $C$ to blind the investigator. Subjects were instructed to take 4 capsules directly after breakfast, lunch and dinner. Empty sachets and unused capsules had to be returned, and counted as measure of compliance. Two weeks before the start and during the study, subjects were asked to abstain from foods and products containing algae, such as sushi or seaweed salads.

Subjects visited the university at the start (day 0) and twice at the end of each intervention period (days 14 and 17). They were asked to abstain from alcohol consumption and exercise the day preceding the visits. At each visit, fasting blood samples were taken by venipuncture after an overnight fast of at least 12 hours. In addition, blood pressure and body weight were measured. At the end of each intervention period, subjects were asked to complete a validated food frequency questionnaire to assess food intake over the past two weeks. Energy and nutrient intakes were calculated using the Dutch food composition table (NEVO). Throughout the study, subjects were asked not to change their diets and physical activity patterns, and were instructed to record daily any changes in health status and their potential alcohol consumption in a study diary.

\section{Blood sampling and analysis}

Blood was drawn into serum and sodium fluoride $(\mathrm{NaF})$-containing tubes (Becton, Dickinson and Company, Franklin Lakes, NJ, USA) at each visit. Serum separator tubes were allowed to clot at room temperature for 30-60 minutes after withdrawal. Next, the tubes were centrifuged at $1300 \times \mathrm{g}$ for 15 minutes at $21^{\circ} \mathrm{C}$ to prepare serum. NaF-containing vacutainer tubes were placed on ice immediately after withdrawal and centrifuged at $4{ }^{\circ} \mathrm{C}$ for 15 minutes at $1300 \mathrm{xg}$ to prepare $\mathrm{NaF}$ plasma. Serum and NaF plasma samples were directly frozen in liquid nitrogen and stored 
at $-80^{\circ} \mathrm{C}$ until analysis. For all analysis, all samples from one subject were analyzed in the same analytical run.

Serum plant sterol (campesterol, sitosterol) concentrations, cholestanol, and concentrations of the cholesterol precursor lathosterol were measured in samples collected at the end of each intervention period (days 14 and 17) by GC-FID as previously described. ${ }^{28}$ Values were standardized for total cholesterol concentrations as measured by GC-FID, and expressed as $\mu \mathrm{mol} / \mathrm{mmol}$ total cholesterol.

Serum TC concentrations (CHOD-PAP method; Roche Diagnostics System, Mannheim, Germany), HDL cholesterol (HDL-C) concentrations (precipitation method followed by CHOD-PAP method; Roche Diagnostics System), triacylglycerol concentrations corrected for free glycerol (GPO-Trinder, Sigma Diagnostics, St Louis, USA), high-sensitivity C-reactive protein (hsCRP) concentrations (immunoturbidimetric assay, Horiba ABX, Montpellier, France) and plasma glucose concentrations (Horiba $A B X$ ) were measured in all samples. LDL-C concentrations were calculated using the Friedewald formula. ${ }^{29}$

\section{Blood pressure measurements}

Systolic and diastolic blood pressure was determined after a 5-minute rest in seated position during every visit (Omron M7, Omron Healthcare Co., Ltd, Kyoto, Japan). Four measurements were performed. The first measurement was discarded and the last three measurements were averaged for data analyses.

\section{Statistics}

It was estimated that a sample size of 33 subjects was needed to detect a true difference of $0.24 \mu \mathrm{mol} / \mathrm{mmol}$ in cholesterol-standardized campesterol concentrations with a power of $80 \%$ and a within-subject variability of 0.47 $\mu \mathrm{mol} / \mathrm{mmol}^{30,31}$ This effect size was chosen since earlier studies from our group showed comparable effects using plant stanol supplementation. ${ }^{30-32}$ As the anticipated dropout rate was 10\%, 36 subjects were recruited.

All results are presented as means \pm SDs. Values at the end of the three periods (days 14 and 17) were averaged for all parameters. A priori, it was decided that 
comparisons would only be made between the spirulina and control conditions, and between the wakame and control conditions, and not between the spirulina and wakame conditions. Differences in end-of-intervention values between spirulina or wakame and control conditions were compared using linear mixed models with subject as random factor, and treatment and period as fixed factors. Differences in end-of-intervention hsCRP concentrations were compared using the non-parametric Friedman test. P-values $<0.05$ were considered to be statistically significant. The interaction term treatment * period was used to test for carry-over effects with linear mixed models. However, this interaction term never reached statistical significance and was therefore removed from all models. Data were analyzed separately for men and women and baseline TC concentrations above and below $5.0 \mathrm{mmol} / \mathrm{L}$, but this did not change the conclusions. The spirulina and wakame conditions were each compared with the placebo condition using post-hoc tests. To correct for multiple comparisons, P-values $<0.025$ were then considered statistically significant. Statistical analyses were performed using SPSS 25.0 for Mac (IBM Corp., Armonk, NY, USA).

\section{Results}

\section{Subjects and compliance}

Thirty-six subjects started the intervention and one subject dropped out due to personal reasons (Figure 4.1). In the end, 35 subjects (15 men and 20 women) completed the trial and were included in the statistical analyses. LDL-C data for one subject could not be calculated due to triacylglycerol concentrations above the 4.52 $\mathrm{mmol} / \mathrm{L}$ threshold for reliable use of the Friedewald formula. ${ }^{29}$ 


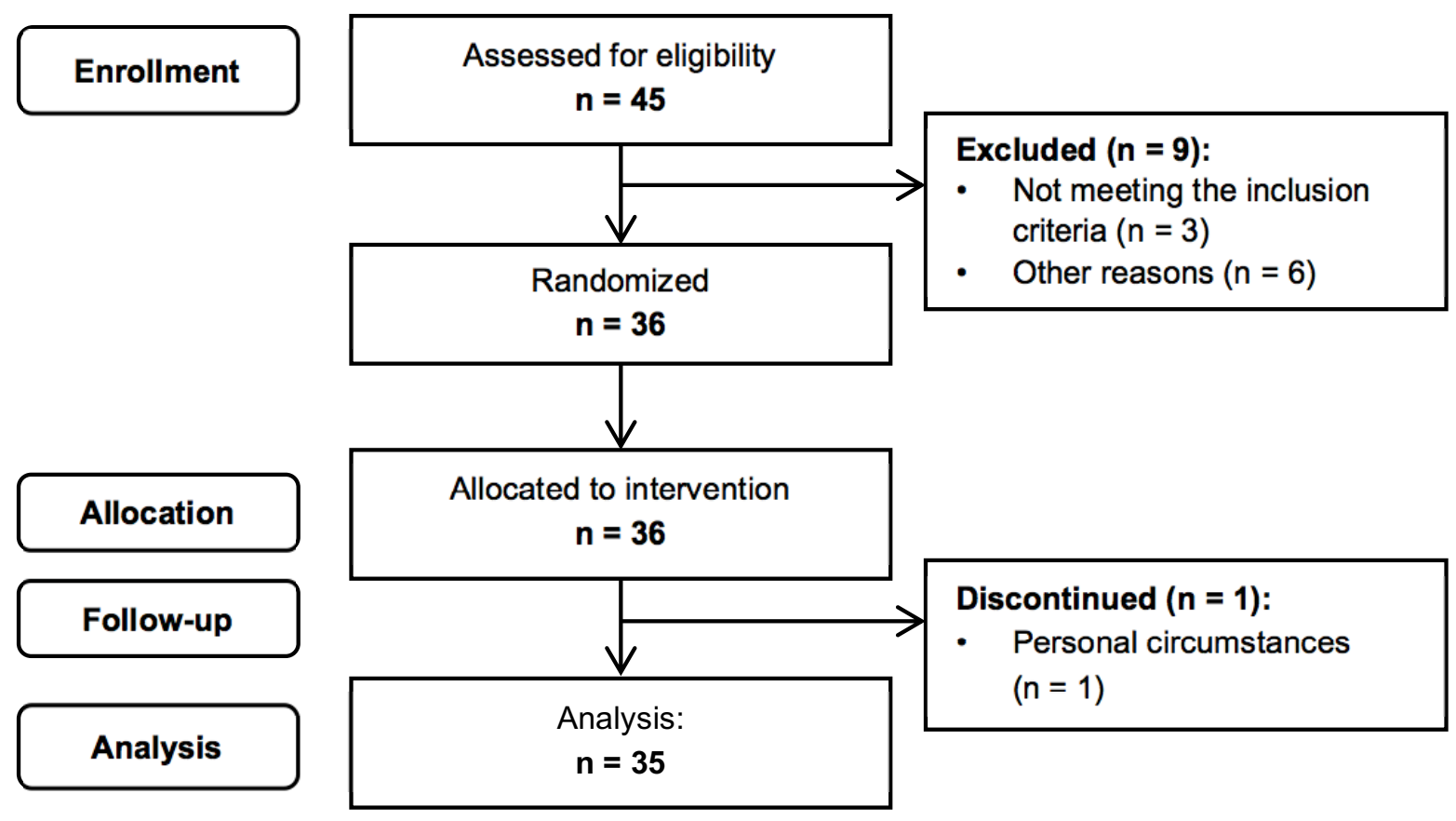

Figure 4.1: flow chart of participants throughout the study

Baseline characteristics of the 35 subjects that completed the trial are shown in

Table 4.1. Changes in weight of the subjects did not differ between the spirulina ($0.2 \pm 0.7 \mathrm{~kg})$, wakame $(-0.1 \pm 0.6 \mathrm{~kg})$ and placebo periods $(-0.2 \pm 0.6 \mathrm{~kg} ; \mathrm{P}=0.925$ for treatment effect). Serum hsCRP concentrations also did not differ between the three intervention periods $(P=0.450)$. Overall compliance was $99 \%(98.2-$ $99.5 \%$ ) based on capsule count.

Table 4.1: Baseline characteristics of men and women who completed the study $(n=35)$

\begin{tabular}{lc}
\hline & Mean \pm SD \\
\hline Men / women, $\mathrm{n}$ & $15 / 20$ \\
\hline Age $(\mathrm{y})$ & $40.2 \pm 19.6$ \\
\hline $\mathrm{BMI}\left(\mathrm{kg} / \mathrm{m}^{2}\right)$ & $24.7 \pm 2.7$ \\
\hline Weight $(\mathrm{kg})$ & $71.9 \pm 12.1$ \\
\hline Total cholesterol $(\mathrm{mmol} / \mathrm{L})$ & $4.9 \pm 1.1$ \\
\hline HDL cholesterol $(\mathrm{mmol} / \mathrm{L})$ & $1.7 \pm 0.5$ \\
\hline LDL cholesterol $(\mathrm{mmol} / \mathrm{L})$ & $2.7 \pm 1.0$ \\
\hline Triacylglycerol $(\mathrm{mmol} / \mathrm{L})$ & $1.1 \pm 0.6$ \\
\hline Glucose $(\mathrm{mmol} / \mathrm{L})$ & $5.2 \pm 1.0$ \\
\hline
\end{tabular}




\section{Dietary intake}

Average daily intakes of energy and the macronutrients did not differ between the three intervention periods (Table 4.2). In addition, cholesterol and fiber intakes were also not different.

Table 4.2: Dietary intake as assessed with food frequency questionnaires after spirulina, wakame and placebo intake

\begin{tabular}{lccc}
\hline & Spirulina & Wakame & Placebo \\
\hline Energy (MJ/day) & $9.1 \pm 2.4$ & $8.7 \pm 2.8$ & $9.2 \pm 2.9$ \\
\hline Fat (energy \%) & $37.6 \pm 7.6$ & $37.9 \pm 7.6$ & $39.2 \pm 8.6$ \\
SFA & $12.7 \pm 3.9$ & $12.7 \pm 3.3$ & $13.2 \pm 3.4$ \\
MUFA & $14.1 \pm 3.5$ & $14.4 \pm 3.4$ & $14.9 \pm 4.0$ \\
PUFA & $7.3 \pm 2.3$ & $7.2 \pm 2.3$ & $7.6 \pm 2.9$ \\
\hline Protein (energy \%) & $16.3 \pm 3.1$ & $16.7 \pm 3.3$ & $16.4 \pm 3.3$ \\
\hline Carbohydrates (energy \%) & $40.5 \pm 7.1$ & $39.9 \pm 8.0$ & $39.1 \pm 8.7$ \\
\hline Alcohol (energy \%) & $3.0 \pm 2.6$ & $3.0 \pm 2.2$ & $2.8 \pm 2.4$ \\
\hline Fiber (g/day) & $26.3 \pm 5.5$ & $24.4 \pm 7.9$ & $24.2 \pm 5.6$ \\
\hline Cholesterol (mg/day) & $238 \pm 113$ & $224 \pm 124$ & $256 \pm 160$ \\
\hline
\end{tabular}

SFA: saturated fatty acids; MUFA: monounsaturated fatty acids; PUFA: polyunsaturated fatty acids

\section{Serum plant sterols, cholestanol and lathosterol concentrations}

Concentrations of cholesterol-standardized serum campesterol, sitosterol and cholestanol, markers for intestinal cholesterol absorption, did not differ between the spirulina and placebo conditions $(P=0.435, P=0.314, P=0.610$, respectively), or the wakame and placebo conditions $(P=0.729, P=0.112, P=0.809$, respectively; Table 4.3). Serum cholesterol-standardized lathosterol concentrations, a marker for cholesterol synthesis, did also not differ between the spirulina or wakame and placebo conditions $(P=0.388$ and $P=0.102$, respectively).

\section{Serum lipids}

Serum lipid concentrations are shown in Table 4.3. No differences were found between the spirulina and placebo conditions for serum total cholesterol $(P=0.443)$, LDL-C ( $P=0.677)$, HDL-C $(P=0.273)$ and triacylglycerol concentrations $(P=0.684)$. Serum total cholesterol $(P=0.749)$, LDL-C $(P=0.902)$, HDL-C $(P=0.937)$, and 
triacylglycerol concentrations $(P=0.302)$ did also not differ between the wakame and placebo conditions. When subjects were divided into the $50 \%$ highest and $50 \%$ lowest 'cholesterol absorbers' based on the median lathosterol to campesterol ratio, ${ }^{33}$ still no differences were found between the spirulina or wakame versus the control conditions within the two subgroups (Supplemental table 4.2).

Table 4.3: Serum cholesterol-standardized concentrations of plant sterols and lathosterol, and lipid concentrations after spirulina, wakame and placebo intake $(n=35)$

\begin{tabular}{|c|c|c|c|c|c|}
\hline & \multirow[t]{2}{*}{ Spirulina } & \multirow[t]{2}{*}{ Wakame } & \multirow[t]{2}{*}{ Placebo } & \multicolumn{2}{|c|}{$\begin{array}{c}\text { Estimated difference } \\
\text { (versus placebo) }^{a}\end{array}$} \\
\hline & & & & Spirulina & Wakame \\
\hline $\begin{array}{l}\text { Campesterol } \\
(\mu \mathrm{mol} / \mathrm{mmol})\end{array}$ & $2.60 \pm 1.11$ & $2.69 \pm 1.11$ & $2.66 \pm 0.99$ & $\begin{array}{c}-0.07 \\
(-0.23-0.10)\end{array}$ & $\begin{array}{c}0.03 \\
(-0.14-0.19)\end{array}$ \\
\hline $\begin{array}{l}\text { Sitosterol } \\
(\mu \mathrm{mol} / \mathrm{mmol})\end{array}$ & $2.25 \pm 0.76$ & $2.42 \pm 0.75$ & $2.32 \pm 0.67$ & $\begin{array}{c}-0.07 \\
(-0.19-0.06)\end{array}$ & $\begin{array}{c}0.10 \\
(-0.03-0.23)\end{array}$ \\
\hline $\begin{array}{l}\text { Cholestanol } \\
(\mu \mathrm{mol} / \mathrm{mmol})\end{array}$ & $1.51 \pm 0.33$ & $1.52 \pm 0.31$ & $1.52 \pm 0.33$ & $\begin{array}{c}-0.01 \\
(-0.06-0.03)\end{array}$ & $\begin{array}{c}-0.01 \\
(-0.05-0.04)\end{array}$ \\
\hline $\begin{array}{l}\text { Lathosterol } \\
(\mu \mathrm{mol} / \mathrm{mmol})\end{array}$ & $1.58 \pm 0.52$ & $1.62 \pm 0.50$ & $1.54 \pm 0.50$ & $\begin{array}{c}0.04 \\
(-0.05-0.13)\end{array}$ & $\begin{array}{c}0.08 \\
(-0.02-0.17)\end{array}$ \\
\hline $\begin{array}{l}\text { Total cholesterol } \\
(\mathrm{mmol} / \mathrm{L})\end{array}$ & $4.75 \pm 1.00$ & $4.84 \pm 1.02$ & $4.81 \pm 1.09$ & $\begin{array}{c}-0.06 \\
(-0.22-0.10)\end{array}$ & $\begin{array}{c}0.03 \\
(-0.13-0.18)\end{array}$ \\
\hline $\begin{array}{l}\text { LDL cholesterol } \\
(\mathrm{mmol} / \mathrm{L})^{b}\end{array}$ & $2.75 \pm 0.97$ & $2.78 \pm 1.04$ & $2.77 \pm 1.07$ & $\begin{array}{c}-0.03 \\
(-0.15-0.10)\end{array}$ & $\begin{array}{c}0.01 \\
(-0.12-0.13)\end{array}$ \\
\hline $\begin{array}{l}\text { HDL cholesterol } \\
(\mathrm{mmol} / \mathrm{L})\end{array}$ & $1.52 \pm 0.43$ & $1.56 \pm 0.42$ & $1.56 \pm 0.49$ & $\begin{array}{c}-0.04 \\
(-0.11-0.03)\end{array}$ & $\begin{array}{c}0.00 \\
(-0.07-0.07)\end{array}$ \\
\hline $\begin{array}{l}\text { Triacylglycerol } \\
\text { (mmol/L) }\end{array}$ & $1.09 \pm 0.63$ & $1.12 \pm 0.80$ & $1.06 \pm 0.61$ & $\begin{array}{c}0.02 \\
(-0.09-0.14)\end{array}$ & $\begin{array}{c}0.06 \\
(-0.06-0.18)\end{array}$ \\
\hline
\end{tabular}

\footnotetext{
${ }^{a}$ Estimated difference and $95 \%$ confidence interval $(\mathrm{Cl})$, based on estimated marginal means obtained with linear mixed models

${ }^{\mathrm{b}} \mathrm{n}=34$ for LDL cholesterol concentrations
}

\section{Glucose concentrations and blood pressure}

No differences were found between the spirulina and placebo conditions for plasma glucose concentrations $(P=0.375)$, as well as between the wakame and placebo conditions $(P=0.373)$. Systolic and diastolic blood pressure did also not differ 
between the spirulina and placebo $(P=0.651$ and $P=0.550$, respectively; Table 4.4), or the wakame and placebo conditions $(P=0.620$ and $P=0.677$, respectively).

Table 4.4: Plasma glucose concentrations, and systolic and diastolic blood pressures after spirulina, wakame and placebo consumption $(n=35)$

\begin{tabular}{lccccc}
\hline & \multirow{2}{*}{ Spirulina } & Wakame & Placebo & \multicolumn{2}{c}{$\begin{array}{c}\text { Estimated difference } \\
\text { (versus placebo) }\end{array}$} \\
\cline { 5 - 6 } & & & Spirulina & Wakame \\
\hline Glucose $(\mathrm{mmol} / \mathrm{L})$ & $5.27 \pm 0.37$ & $5.27 \pm 0.39$ & $5.23 \pm 0.37$ & $\begin{array}{c}0.04 \\
(-0.04-\end{array}$ & 0.04 \\
& & & & $0.11)$ & $(-0.04-0.11)$ \\
\hline $\begin{array}{l}\text { Systolic blood } \\
\text { pressure }(\mathrm{mmHg})\end{array}$ & $113.9 \pm 13.7$ & $114.1 \pm 14.3$ & $114.4 \pm 14.5$ & -0.5 & -0.6 \\
\hline $\begin{array}{l}\text { Diastolic blood } \\
\text { pressure }(\mathrm{mmHg})\end{array}$ & $75.4 \pm 9.4$ & $75.3 \pm 9.3$ & $74.9 \pm 9.5$ & $\begin{array}{c}0.5 \\
(-1.1-2.8-1.8)\end{array}$ & $(-2.9-1.7)$ \\
\hline
\end{tabular}

${ }^{\text {a }}$ Estimated difference and 95\% confidence interval $(\mathrm{Cl})$, based on estimated marginal means obtained with linear mixed models

\section{Discussion}

In this placebo-controlled double-blind intervention study, daily consumption of 4.8 $\mathrm{g}$ spirulina or $4.8 \mathrm{~g}$ wakame for 17 days did not affect markers for intestinal cholesterol absorption and endogenous cholesterol synthesis in nonhypercholesterolemic healthy men and women. In agreement, serum lipid concentrations were also not affected. Also, no effects on plasma glucose concentrations and blood pressure were observed.

Animal studies have suggested that spirulina and wakame consumption inhibit intestinal cholesterol absorption. In male Wistar rats, a spirulina concentrate increased fecal steroid content with a concomitant decrease in LDL-C concentrations. ${ }^{9}$ In two in vitro experiments, a spirulina concentrate decreased micellar solubility of cholesterol and suppressed cholesterol absorption in Caco-2 cells. Similarly, supplementation with wakame or a wakame extract increased fecal cholesterol excretion in male Wistar rats ${ }^{19}$ and C57BL/6J mice on a high-fat diet, ${ }^{21}$ again suggesting inhibition of intestinal cholesterol absorption. However, since serum cholesterol-standardized campesterol, sitosterol and cholestanol 
concentrations were not changed, our results do not suggest that these two algae did have an effect on intestinal cholesterol absorption in humans. In addition, cholesterol-standardized lathosterol concentrations, a marker reflecting endogenous cholesterol synthesis, were not altered. The use of serum noncholesterol sterol and stanol concentrations as markers for intestinal cholesterol absorption and endogenous cholesterol synthesis has been well validated. ${ }^{34}$ Yet, when the intake of these sterols changes, plasma levels do not reflect cholesterol absorption anymore. However, levels of sterols in the algae were very low (Supplemental Table 4.1) and therefore did not affect the validity of plasma plant sterols as markers for intestinal cholesterol absorption. In addition, cholestanol was not present in the algae and the observation that serum cholestanol concentrations were not affected confirmed the lack of an effect on intestinal cholesterol absorption.

As expected by the lack of effects on intestinal cholesterol absorption and endogenous cholesterol synthesis, serum TC or LDL-C concentrations were also unchanged. This contrasts findings from a recent meta-analysis, including 10 RCTs with 12 treatment arms and more than 700 subjects, evaluating the effects of spirulina consumption on serum lipid concentrations. ${ }^{27}$ Decreases of $-1.00 \mathrm{mmol} / \mathrm{L}$ and $-0.91 \mathrm{mmol} / \mathrm{L}$ were reported for TC and LDL-C respectively. These effects are large for a dietary intervention and are in the range of those achieved with drugs. ${ }^{35}$ Although the present study was not primarily powered on changes in LDL-C, posthoc calculations showed that the statistical power of our study was close to $100 \%$ to pick up such an effect. In the same meta-analysis, a decrease in triacylglycerol concentrations was found, whereas those of HDL-C were not significantly changed. However, 5 of the 10 RCTs measuring serum lipid concentrations were not blinded, since the control groups received no placebo capsules or tablets. ${ }^{10,12-14,36}$ In fact, subgroup analysis revealed that TC, LDL-C, TAG concentrations decreased and those of HDL-C increased in the trials with a no-intervention control group. When a placebo group was included, only TC concentrations decreased. Differences in dose, duration of the intervention and study populations are factors to explore in trying to explain discrepancies in results.

Intake of spirulina differed largely between the 10 trials included in the metaanalysis ranging from 1 to 19 grams daily, with a median intake of 2 grams. $^{27}$ 
Subgroup analysis suggested that lipid-lowering effects were found with consumption of 2 grams or more, whereas no significant effects were found with intakes less than 2 grams a day. As our daily dose of 4.8 grams is clearly above this median intake of 2 grams, it is unlikely that differences in spirulina dosage could explain the lack of effects.

The duration of our intervention was shorter compared to earlier trials. The median intervention duration in the meta-analyses of Huang and colleagues was 12 weeks. ${ }^{27}$ Subgroup analysis revealed that significant changes in lipid concentrations where only found in the 7 RCTs lasting 12 weeks or longer, but only three of them used placebos instead of a no-intervention control group. It is not likely that our shorter study duration can explain the lack of effect on LDL-C, as LDL-C concentrations reach a new steady state within 2 weeks when intestinal cholesterol absorption is inhibited by dietary components or drugs. ${ }^{37,38}$

Our study population also varied from those of other studies. Spirulina lowered TC, LDL-C and TAG, but not HDL-C concentrations in type II diabetics ${ }^{11,39}$ and children with the nephrotic syndrome. ${ }^{14}$ On the other hand, two other studies in type II diabetics only reported TAG-lowering effects and no effects on TC, LDL-C and HDL-C concentrations. ${ }^{10,40}$ Lipid concentrations were all improved in ischemic heart disease patients with hypercholesterolemia, whereas no effects on any of the lipid parameters were seen in obese subjects. ${ }^{15}$ In HIV-patients ${ }^{12}$ and hypertensive subjects, ${ }^{41} \mathrm{TC}$ and LDL-C concentrations decreased and those of HDL-C increased. In elderly, only TC concentrations were decreased. ${ }^{42}$ Overall, heterogeneity between studies was large and there was no evidence that some populations were more responsive than others. It is therefore not likely that effects of spirulina consumption are only evident in subjects with increased baseline total cholesterol concentrations and not in our healthy, non-hypercholesterolemic population. Also, studies with plant sterols and stanols have demonstrated LDL-C lowering effects via inhibition of intestinal cholesterol absorption in non-hypercholesterolemic subjects. ${ }^{32,37}$ In conclusion, there is no clear reason why our results do not support the results of the meta-analysis of Huang et al. ${ }^{27}$ Possibly, lack of blinding of some of the earlier studies may have biased outcomes. 
Results of human trials investigating the effect of wakame consumption on TC or LDL-C concentrations are more in line with our results in non-hypercholesterolemic subjects. No effects were found in hypertensive subjects, ${ }^{22}$ subjects with the metabolic syndrome,$^{23}$ and HIV patients. ${ }^{24}$ In former trials daily intakes ranged between 4 to 6 grams, which is comparable to the intake used in our study. In one study, $500 \mathrm{mg}$ of fucoidan extracted from brown seaweed lowered LDL-C concentrations in overweight and obese subjects. ${ }^{25}$ However, this amount of fucoidan is present in $13-46$ grams of wakame, ${ }^{43}$ which is much higher than the amount of 4.8 grams provided in our and the other studies. Whether the wakameextract fucoidan truly lowers LDL-C warrants further study.

Glucose concentrations and blood pressure were assessed as additional markers for CVD risk, but were not changed by spirulina or wakame consumption. A metaanalysis including eight RCTs suggested glucose-lowering effects of spirulina consumption, ${ }^{27}$ which is in contrast with our results. No subgroup analyses were performed. Of the 8 studies included, 4 were certainly not blinded. Decreases in fasting glucose concentrations were observed in studies with type II diabetics, ${ }^{11,36,39}$ HIV patients, ${ }^{44}$ and hypertensive subjects. ${ }^{41}$ However, in two other trials with type II diabetics ${ }^{10,40}$ and a trial with children with the nephrotic syndrome, ${ }^{14}$ glucose concentrations were not affected. None of the trials with wakame reported effects on glucose concentrations. ${ }^{23,24}$ Diastolic blood pressure was also significantly lowered after spirulina consumption in the recent meta-analysis, ${ }^{34}$ whereas systolic blood pressure was not affected. Three studies were included in the analysis, of which 2 were blinded. However, in only one individual trial with hypertensive subjects, spirulina consumption significantly affected diastolic blood pressure. ${ }^{45}$ Wakame consumption did affect systolic and diastolic blood pressure in hypertensive subjects $^{22}$ and systolic blood pressure in subjects with the metabolic syndrome. ${ }^{23}$ In the latter trial, effects were only present in a hypertensive subgroup. Thus, it may be that algae consumption only lowers blood pressure in subjects with increased baseline blood pressure levels. Although this needs to be explored further, it might explain the lack of an effect on blood pressure in our trial with non-hypertensive subjects. 
To conclude, our study indicates that consuming 4.8 grams/day spirulina or wakame for 17 days does not inhibit intestinal cholesterol absorption in nonhypercholesterolemic men and women, nor does it affect lipid profiles. In addition, blood pressure and glucose concentrations were not affected by spirulina or wakame consumption.

\section{Acknowledgements}

The authors would like to thank Maud Beckers and Lynn Vrenken for technical and dietary support throughout the study. We thank Dieter Lutjohann and Kenneth Vanbrabant from Bonn University for analyzing the sterol composition from spirulina and wakame. 


\section{References}

1. Cholesterol Treatment Trialists' (CTT) Collaboration. The effects of lowering LDL cholesterol with statin therapy in people at low risk of vascular disease: meta-analysis of individual data from 27 randomised trials. Lancet. 2012;380(9841): 581-590

2. Cohen DE. Balancing cholesterol synthesis and absorption in the gastrointestinal tract. J Clin Lipidol. 2008;2(2):S1-S3.

3. Brown L, Rosner B, Willett WW, Sacks FM. Cholesterol-lowering effects of dietary fiber: a metaanalysis. Am J Clin Nutr. 1999;69(1):30-42.

4. Feuerstein JS, Bjerke WS. Powdered red yeast rice and plant stanols and sterols to lower cholesterol. J Diet Suppl. 2012;9(2):110-5.

5. Van den Driessche JJ, Plat J, Mensink RP. Effects of superfoods on risk factors of metabolic syndrome: a systematic review of human intervention trials. Food Funct. 2018;9(4):1944-66.

6. Wells ML, Potin P, Craigie JS, Raven JA, Merchant SS, Helliwell KE, et al. Algae as nutritional and functional food sources: revisiting our understanding. J Appl Phycol. 2017;29(2):949-82.

7. Buono S, Langellotti AL, Martello A, Rinna F, Fogliano V. Functional ingredients from microalgae. Food Funct. 2014;5(8):1669-85.

8. Iwata K, Inayama T, Kato T. Effects of Spirulina platensis on plasma lipoprotein lipase activity in fructose-induced hyperlipidemic rats. J Nutr Sci Vitaminol. 1990;36(2):165-71.

9. Nagaoka S, Shimizu K, Kaneko H, Shibayama F, Morikawa K, Kanamaru Y, et al. A novel protein C-phycocyanin plays a crucial role in the hypocholesterolemic action of Spirulina platensis concentrate in rats. J Nutr. 2005;135(10):2425-30.

10. Lee EH, Park JE, Choi YJ, Huh KB, Kim WY. A randomized study to establish the effects of spirulina in type 2 diabetes mellitus patients. Nutr Res Pract. 2008;2(4):295-300.

11. Mani UV, Desai S, Iyer U. Studies on the Long-Term Effect of Spirulina supplementation on serum lipid profile and glycated proteins in NIDDM patients. J Diet Suppl. 2000;2(3):25-32.

12. Ngo-Matip ME, Pieme CA, Azabji-Kenfack M, Biapa PC, Germaine N, Heike E, et al. Effects of Spirulina platensis supplementation on lipid profile in HIV-infected antiretroviral naive patients in Yaounde-Cameroon: a randomized trial study. Lipids Health Dis. 2014;13:191.

13. Ramamoorthy A, Premakumari S. Effect of supplementation of Spirulina on hypercholesterolemic patients. J Food Sci Technol. 1996;33(2):124-8.

14. Samuels R, Mani UV, Iyer UM, Nayak US. Hypocholesterolemic effect of spirulina in patients with hyperlipidemic nephrotic syndrome. J Med Food. 2002;5(2):91-6.

15. Zeinalian R, Farhangi MA, Shariat A, Saghafi-AsI M. The effects of Spirulina Platensis on anthropometric indices, appetite, lipid profile and serum vascular endothelial growth factor (VEGF) in obese individuals: a randomized double blinded placebo controlled trial. BMC Complementary Altern Med. 2017;17(1):225.

16. Zava TT, Zava DT. Assessment of Japanese iodine intake based on seaweed consumption in Japan: A literature-based analysis. Thyroid Res. 2011;4:14. 
17. Zhang H, Pang Z, Han C. Undaria pinnatifida (Wakame): a seaweed with pharmacological properties. Sci Int. 2014;2(2):32-6.

18. Grasa-López A, Miliar-García Á, Quevedo-Corona L, Paniagua-Castro N, Escalona-Cardoso G, Reyes-Maldonado $\mathrm{E}$, et al. Undaria pinnatifida and fucoxanthin ameliorate lipogenesis and markers of both inflammation and cardiovascular dysfunction in an animal model of diet-induced obesity. Mar Drugs. 2016;14(8):148.

19. Iritani N, Nogi J. Effect of spinach and wakame on cholesterol turnover in the rat. Atherosclerosis. 1972;15(1):87-92.

20. Yoshinaga K, Nakai Y, Izumi H, Nagaosa K, Ishijima T, Nakano T, et al. Oral administration of edible seaweed Undaria pinnatifida (Wakame) modifies glucose and lipid metabolism in rats: a DNA microarray analysis. Mol Food Res. 2018;62(12):e1700828.

21. Jeon SM, Kim HJ, Woo MN, Lee MK, Shin YC, Park YB, et al. Fucoxanthin-rich seaweed extract suppresses body weight gain and improves lipid metabolism in high-fat-fed C57BL/6J mice. Biotechnol J. 2010;5(9):961-9.

22. Hata $\mathrm{Y}$, Nakajima K, Uchida J-i, Hidaka H, Nakano T. Clinical effects of brown seaweed, Undaria pinnatifida (wakame), on blood pressure in hypertensive subjects. J Clin Biochem Nutr. 2001;30:4353.

23. Teas J, Baldeon ME, Chiriboga DE, Davis JR, Sarries AJ, Braverman LE. Could dietary seaweed reverse the metabolic syndrome? Asia Pac J Clin Nutr. 2009;18(2):145-54.

24. Teas J, Irhimeh MR. Dietary algae and HIVIAIDS: proof of concept clinical data. J Appl Phycol. 2012;24(3):575-82.

25. Hernandez-Corona DM, Martinez-Abundis E, Gonzalez-Ortiz M. Effect of fucoidan administration on insulin secretion and insulin resistance in overweight or obese adults. J Med Food. 2014;17(7):830-2.

26. Alberti KG, Eckel RH, Grundy SM, Zimmet PZ, Cleeman JI, Donato KA, et al. Harmonizing the metabolic syndrome: a joint interim statement of the International Diabetes Federation Task Force on Epidemiology and Prevention; National Heart, Lung, and Blood Institute; American Heart Association; World Heart Federation; International Atherosclerosis Society; and International Association for the Study of Obesity. Circulation. 2009;120(16):1640-5.

27. Huang $H$, Liao $D, P u R$, Cui $Y$. Quantifying the effects of spirulina supplementation on plasma lipid and glucose concentrations, body weight, and blood pressure. Diabetes, Metab Syndr, Obes Targets Ther. 2018;11:729-42.

28. Teunissen CE, Mulder M, de Vente J, von Bergmann K, De Bruijn C, Steinbusch HW, et al. Concentrations of different sterols in the striatum and serum of 3-nitropropionic acid-treated Wistar and Lewis rats. Neurochem Res. 2001;26(11):1237-44.

29. Friedewald WT, Levy RI, Fredrickson DS. Estimation of the concentration of low-density lipoprotein cholesterol in plasma, without use of the preparative ultracentrifuge. Clin Chem. 1972;18(6):499502. 
30. Brull F, De Smet E, Mensink RP, Vreugdenhil A, Kerksiek A, Lutjohann D, et al. Dietary plant stanol ester consumption improves immune function in asthma patients: results of a randomized, doubleblind clinical trial. Am J Clin Nutr. 2016;103(2):444-53.

31. Mensink RP, de Jong A, Lutjohann D, Haenen GR, Plat J. Plant stanols dose-dependently decrease LDL-cholesterol concentrations, but not cholesterol-standardized fat-soluble antioxidant concentrations, at intakes up to $9 \mathrm{~g} / \mathrm{d}$. Am J Clin Nutr. 2010;92(1):24-33.

32. Baumgartner S, Mensink RP, Husche C, Lutjohann D, Plat J. Effects of plant sterol- or stanolenriched margarine on fasting plasma oxyphytosterol concentrations in healthy subjects. Atherosclerosis. 2013;227(2):414-9.

33. Thuluva SC, Igel M, Giesa U, Lutjohann D, Sudhop T, von Bergmann K. Ratio of lathosterol to campesterol in serum predicts the cholesterol-lowering effect of sitostanol-supplemented margarine. Int J Clin Pharmacol Ther. 2005;43(7):305-10.

34. Miettinen TA, Tilvis RS, Kesaniemi YA. Serum plant sterols and cholesterol precursors reflect cholesterol absorption and synthesis in volunteers of a randomly selected male population. Am J Epidemiol. 1990;131(1):20-31.

35. Ohta A, Kato H, Ishii S, Nagai Y, Tanaka Y. Effect of ezetimibe monotherapy on low-density lipoprotein cholesterol and on markers of cholesterol synthesis and absorption in Japanese patients with hypercholesterolemia. J Clin Med Res. 2017;9(6):476-81.

36. Anitha L, Chandralekha K. Effect of supplementation of Spirulina on blood glucose, glycosylated hemoglobin and lipid profile of male non-insulin dependent diabetics. Asian J Exp Biol Sci. 2010;1(1):36-46.

37. Mensink RP, Ebbing S, Lindhout M, Plat J, van Heugten MM. Effects of plant stanol esters supplied in low-fat yoghurt on serum lipids and lipoproteins, non-cholesterol sterols and fat soluble antioxidant concentrations. Atherosclerosis. 2002;160(1):205-13.

38. Knopp RH, Gitter H, Truitt T, Bays H, Manion CV, Lipka LJ, et al. Effects of ezetimibe, a new cholesterol absorption inhibitor, on plasma lipids in patients with primary hypercholesterolemia. Eur Heart J. 2003;24(8):729-41.

39. Kamalpreet K, Rajbir S, Kiran G. Effect of supplementation of Spirulina on blood glucose and lipid profile of the non-insulin dependent diabetic male subjects. Asian $\mathrm{J}$ Dairy Res. 2008;27(3and4):202-8.

40. Parikh P, Mani U, lyer U. Role of Spirulina in the control of glycemia and lipidemia in type 2 diabetes mellitus. J Med Food. 2001;4(4):193-9.

41. Szulinska M, Gibas-Dorna M, Miller-Kasprzak E, Suliburska J, Miczke A, Walczak-Galezewska M, et al. Spirulina maxima improves insulin sensitivity, lipid profile, and total antioxidant status in obese patients with well-treated hypertension: a randomized double-blind placebo-controlled study. Riv Eur Sci Med Farmacol. 2017;21(10):2473-81.

42. Park HJ, Lee YJ, Ryu HK, Kim MH, Chung HW, Kim WY. A randomized double-blind, placebocontrolled study to establish the effects of spirulina in elderly Koreans. Ann Nutr Metab. 2008;52(4):322-8. 
43. Lee YK, Lim D-J, Lee Y-H, Park Y-I. Variation in fucoidan contents and monosaccharide compositions of korean Undaria pinnatifida (Harvey) Suringar (Phaeophyta). Algae. 2006;21(1):157-60.

44. Ngo-Matip M-E, Pieme CA, Azabji-Kenfack M, Moukette BM, Korosky E, Stefanini P, et al. Impact of daily supplementation of Spirulina platensis on the immune system of naïve HIV-1 patients in Cameroon: a 12-months single blind, randomized, multicenter trial. Nutr J. 2015;14:70-.

45. Miczke A, Szulinska M, Hansdorfer-Korzon R, Kregielska-Narozna M, Suliburska J, Walkowiak J, et al. Effects of spirulina consumption on body weight, blood pressure, and endothelial function in overweight hypertensive Caucasians: a double-blind, placebo-controlled, randomized trial. Riv Eur Sci Med Farmacol. 2016;20(1):150-6. 


\section{Supplemental materials}

Supplemental table 4.1: Sterol composition of the spirulina and wakame supplements as determined by gas-chromatography flame-ionization-detection (GC-FID) by Bonn University

\begin{tabular}{lcccc}
\hline & \multicolumn{2}{c}{ Spirulina } & \multicolumn{2}{c}{ Wakame } \\
\hline & $\mathbf{n g} / \mathbf{m g}$ & $\boldsymbol{\mu g} /$ day & $\mathbf{n g} / \mathbf{m g}$ & $\boldsymbol{\mu g} /$ day \\
\hline Cholesterol & 7.8 & 37.4 & - & - \\
\hline 24-methyl cholesterol & 7.7 & 37.0 & 65.1 & 312.5 \\
\hline Campesterol & 4.9 & 23.5 & - & - \\
\hline Sitosterol & 19.3 & 92.6 & - & - \\
\hline Stigmasterol & 11.5 & 55.2 & - & - \\
\hline Fucosterol & - & & 476.6 & 2287.7 \\
\hline 24 R/S saringosterol & - & & 17.4 & 83.5 \\
\hline
\end{tabular}

Supplemental table 4.2: Lipid concentrations after spirulina, wakame and placebo intake in the $50 \%$ highest absorbers $(n=17)$ and the $50 \%$ lowest absorbers $(n=18)$

\begin{tabular}{lcccccccc}
\hline & \multicolumn{3}{c}{ Highest cholesterol absorbers } & \multicolumn{3}{c}{ Lowest cholesterol absorbers } \\
& Spirulina & Wakame & Placebo & $\mathrm{P}^{\mathrm{a}}$ & Spirulina & Wakame & Placebo & $\mathrm{P}^{\mathrm{a}}$ \\
\hline TC & $4.28 \pm$ & $4.42 \pm$ & $4.48 \pm$ & 0.269 & $5.13 \pm$ & $5.24 \pm$ & $5.11 \pm$ & 0.645 \\
$(\mathrm{mmol} / \mathrm{L})$ & 0.76 & 0.89 & 0.84 & & 1.04 & 1.02 & 1.26 & \\
\hline $\mathrm{LDL}-\mathrm{C}$ & $2.24 \pm$ & $2.33 \pm$ & $2.34 \pm$ & 0.575 & $3.26 \pm$ & $3.24 \pm$ & $3.21 \pm$ & 0.871 \\
$(\mathrm{mmol} / \mathrm{L})$ & 0.57 & 0.75 & 0.73 & & 0.83 & 0.92 & 1.01 & \\
\hline $\mathrm{HDL}-\mathrm{C}$ & $1.65 \pm$ & $1.65 \pm$ & $1.72 \pm$ & 0.429 & $1.43 \pm$ & $1.52 \pm$ & $1.42 \pm$ & 0.119 \\
$(\mathrm{mmol} / \mathrm{L})$ & 0.42 & 0.43 & 0.51 & & 0.39 & 0.36 & 0.41 & \\
\hline TAG & $0.86 \pm$ & $0.96 \pm$ & $0.93 \pm$ & 0.422 & $1.16 \pm$ & $1.07 \pm$ & $1.03 \pm$ & 0.488 \\
$(\mathrm{mmol} / \mathrm{L})$ & 0.34 & 0.46 & 0.35 & & 0.46 & 0.48 & 0.39 & \\
\hline
\end{tabular}

TC: total cholesterol; LDL-C: LDL cholesterol; HDL-C: HDL cholesterol; TAG: triacylglycerol

${ }^{\text {a }} \mathrm{P}$-value for the treatment effect

${ }^{\mathrm{b}} \mathrm{n}=17$ in the 'lowest cholesterol absorbers' for LDL cholesterol 


\section{CHAPTER 5}

Spirulina, wakame or goji berries do not lower markers of low-grade systemic inflammation in healthy subjects

José J. van den Driessche, Ronald P. Mensink, Jogchum Plat 


\section{Abstract}

Background: We have earlier reported that consumption of the algae spirulina (Arthrospira platensis or maxima) and wakame (Undaria pinnatifida) for 17 days as well as a single dose of goji berries did not affect fasting or postprandial CVD risk markers in non-hypercholesterolemic subjects. However, evidence is increasing that decreasing low-grade systemic inflammation lowers CVD risk. Therefore, we have now examined effects of these algae and of goji berries on markers of low-grade systemic inflammation.

Method: Two randomized, placebo-controlled, crossover trials were performed. In the algae study, 35 non-hypercholesterolemic, healthy subjects consumed 4.8 grams of spirulina, wakame or placebo for 17 days, separated by 14-day washout periods. After 17 days, fasting serum TNFa, IL-6, IL-8, and hsCRP concentrations were measured. In the goji berry study, 17 healthy, overweight men received a mixed meal with or without 25 grams of dried goji berries. Before and up to 4 hours after meal intake, serum concentrations of TNFa, IL-6 and IL-8 were measured.

Results: Consumption of spirulina or wakame did not affect serum concentrations of TNFa, IL-6, IL-8 or hsCRP. In the goji berry study, serum IL-6 and IL-8 concentrations increased postprandially. For IL-8, these increases were more pronounced after the goji berry meal compared to the control meal $(P=0.003)$. No effects on TNFa were observed.

Conclusions: Consumption of spirulina or wakame for 17 days did not affect serum markers of low-grade systemic inflammation. A single dose of goji berries increased postprandial IL-8 concentrations compared to placebo, whereas no effects were found on other markers of low-grade systemic inflammation. 


\section{Introduction}

Dyslipidemia, hyperglycemia, hypertension and obesity are well-known risk factors for cardiovascular diseases (CVDs). ${ }^{1,2}$ In addition, evidence is accumulating that postprandial hyperlipidemia and hyperglycemia also contribute to an elevated risk. ${ }^{3,4}$ Last, markers of low-grade systemic inflammation are associated with CVD risk independent of the classical risk factors. ${ }^{5}$ In fact, it has been shown that lowering inflammation reduced the occurrence of CVD events, even if lipid profiles were not affected. ${ }^{6}$ Therefore, it is important to evaluate whether interventions not only affect the classical CVD risk factors, but also those related to low-grade systemic inflammation.

We have recently examined the effects of three different nutritional compounds on classical CVD risk factors. It was found that the consumption of the algae spirulina (Arthrospira platensis or maxima) or wakame (Undaria pinnatifida) by non-hypercholesterolemic men and women for 17 days did not affect serum lipid or plasma glucose concentrations, and blood pressure. ${ }^{7}$ In another study, we examined the acute effects of a single dose of goji berries (Lycium barbarum fruit) on postprandial triacylglycerol and glucose concentrations in healthy, but overweight men. ${ }^{8}$ Again, no effects of the intervention on these parameters could be demonstrated. However, several lines of evidence from cell, animal and human studies have suggested that spirulina, wakame, goji berries or their extracts may reduce markers of low-grade systemic inflammation, ${ }^{9-12}$ but results were not conclusive. ${ }^{13-16}$ Therefore, we decided to examine the effect of the algae spirulina and wakame, or goji berries on markers of low-grade systemic inflammation in wellcontrolled human intervention trials.

\section{Methods}

\section{Study population}

Detailed characteristics of the study populations have been published previously. 7,8 Briefly, 36 healthy, non-hypercholesterolemic men and women participated in the algae study. Main inclusion criteria were: BMI between 18 and $30 \mathrm{~kg} / \mathrm{m}^{2}$, stable body weight ( $\leq 3 \mathrm{~kg}$ weight loss or gain in the past 3 months), no use of medication or 
food supplements known to affect lipid or glucose metabolism or blood pressure, and no elevated fasting serum total cholesterol $(<8.0 \mathrm{mmol} / \mathrm{L}$ ), serum triacylglycerol $(<4.5 \mathrm{mmol} / \mathrm{L})$ and plasma glucose $(<7.0 \mathrm{mmol} / \mathrm{L})$ concentrations as determined during a screening visit. In the goji berry study, eighteen healthy, overweight (BMI $25-30 \mathrm{~kg} / \mathrm{m}^{2}$ ) men participated. Main inclusion criteria were: stable body weight (s $3 \mathrm{~kg}$ weight loss or gain in the past 3 months), no use of anticoagulants or medications known to affect lipid or glucose metabolism, serum triacylglycerol concentrations below $2.2 \mathrm{mmol} / \mathrm{L}$ and no elevated fasting serum total cholesterol (< $8.0 \mathrm{mmol} / \mathrm{L})$ or plasma glucose $(<7.0 \mathrm{mmol} / \mathrm{L})$ concentrations as determined during a screening visit. Both studies were approved by the medical ethical committee of Maastricht University Medical Centre+ (MUMC+) and registered at clinicaltrials.gov as NCT03380611 (algae study) and NCT02779985 (goji berry study).

\section{Study design: algae study}

The algae study had a randomized, placebo-controlled, double blind crossover design and consisted of three intervention periods of 17 days each, separated by washout periods of at least 14 days. During each intervention period, subjects consumed in a random order spirulina, wakame, or placebo capsules. Twelve capsules, each containing $400 \mathrm{mg}$ spirulina (Flora Health, Burnaby, Canada), 400 mg wakame (Swanson Health, Fargo, North Dakota, USA), or $400 \mathrm{mg}$ microcrystalline cellulose (Radboud UMC, Nijmegen, the Netherlands) had to be consumed daily. Subjects were instructed to take 4 capsules directly after breakfast, lunch and dinner. Two weeks before the start of and during the study, subjects were asked to refrain from foods and products containing algae. At the end of each intervention period (day 17), subjects visited the university after an overnight fast of at least 12 hours and fasting blood samples were taken by venipuncture. The day before each visit, subjects were asked to abstain from alcohol consumption and exercise. 


\section{Study design: goji berry study}

The goji berry study had a randomized, double-blind, crossover design with two treatments. During two test days, separated by a washout period of at least 7 days, a postprandial test with a mixed meal was carried out. The day preceding each test day, subjects were asked to abstain from alcohol consumption, exercise and coffee consumption (from 12 PM onwards) and to consume a standardized evening meal. After an overnight fast of at least 12 hours, subjects came to university by public transport or by car on the days of the postprandial tests.

Subjects received in a random order a mixed meal containing 25 grams of dried Lycium barbarum fruit (Superfood.nl, The Netherlands) or a control meal. The Lycium barbarum and control meals were matched for energy content (684 kcal and $683 \mathrm{kcal}$ respectively) and macronutrient composition (55 En\% fat, $32 \mathrm{En} \%$ carbohydrate, $12 \mathrm{En} \%$ protein vs. $55 \mathrm{En} \%$ fat, $33 \mathrm{En} \%$ carbohydrate, $12 \mathrm{En} \%$ protein; Supplemental Table 5.1). Blood was sampled before (T0) and 30 minutes (T30), 60 minutes (T60), 120 minutes (T120), 180 minutes (T180) and 240 minutes (T240) after meal intake via an intravenous catheter.

\section{Blood sampling and analyses}

Blood handling protocols were identical between the algae and goji berry study. Serum separator tubes (Becton, Dickinson and Company, Franklin Lakes, NJ, USA) were used for blood collection. Tubes were allowed to clot for $30-60$ minutes at room temperature and centrifuged at $1300 \times \mathrm{g}$ for 15 minutes at $21^{\circ} \mathrm{C}$. Serum samples were immediately frozen using liquid nitrogen and stored at $-80^{\circ} \mathrm{C}$ until analysis.

Serum concentrations of interleukin 6 (IL-6), interleukin 8 (IL-8) and tumor necrosis factor alpha (TNFa) were measured using a multi-array detection system based on electro-chemiluminescence technology (MesoScaleDiscovery, SECTOR Imager 2400, Gaithersburg, Maryland, USA) at all indicated visits and time points. Serum high-sensitivity C-reactive protein (hsCRP; immunoturbidimetric assay, Horiba ABX, Montpellier, France) concentrations were measured in samples from all indicated visits within the algae study and in baseline (TO) samples within the goji berry study. All samples from a subject were analyzed in the same analytical run. 


\section{Statistics}

Data is presented as mean and standard deviation (SD), unless indicated otherwise. For the algae study, linear mixed models were used to assess differences in end-ofintervention values between the spirulina or wakame with placebo conditions. It was decided a priori that comparisons would only be made between the spirulina and control conditions and between the wakame and control conditions, and not between the spirulina and wakame conditions. Treatment and period were used as fixed factors and subject as random factor. P-values < 0.05 were considered statistically significant. To test for carry-over effects, the interaction term treatment * period was used. As the interaction terms were not significant, they were omitted from all models. The spirulina and wakame conditions were each compared with the placebo condition using post-hoc tests. To correct for multiple comparisons, P-values $<0.025$ were then considered to be statistically significant. Data was analyzed for men and women separately, but no differences in outcomes were found (data not shown).

Within the goji berry study, fasting values between test days were compared using paired-samples T-tests. Changes in postprandial values from baseline were assessed using linear mixed models with diet and time, and the interaction term diet * time as fixed factors, and subjects as random factor. Since the interaction term was not statistically significant, it was omitted from all models. Post hoc tests with Bonferroni correction were used to compare time points to baseline if factor time was significant. The incremental area under the curve (iAUC), defined as the area above baseline values, was calculated with the trapezoidal rule ${ }^{17}$ for the 4 hours after meal intake and compared using paired-samples T-tests. P-values $<0.05$ were considered statistically significant. Statistical analyses were performed with SPSS 25.0 for Mac (IBM Corp., Armonk, NY, USA).

\section{Results}

\section{Subjects}

One of the 36 subjects that started with the algae study dropped out due to personal reasons during the wakame period of the study. Thus, 35 subjects, 15 men and 20 women, completed the trial. Baseline characteristics of these subjects are shown in 
Eighteen men participated in the goji berry study, of which one man was removed from the statistical analyses due to a clear absent postprandial response. Baseline characteristics of the 17 men included in the analyses are shown in Table 5.1. Subjects had a mean age of $59.5 \pm 5.4$ years and a BMl of $27.2 \pm 1.4 \mathrm{~kg} / \mathrm{m}^{2}$.

Table 5.1: Mean ( \pm SD) baseline characteristics of the 35 men and women and 17 men included in the analyses within the algae and goji berry study respectively

\begin{tabular}{lcc}
\hline & $\begin{array}{c}\text { Algae study } \\
(\mathbf{n}=\mathbf{3 5})\end{array}$ & $\begin{array}{c}\text { Goji berry study } \\
(\mathbf{n}=\mathbf{1 7})\end{array}$ \\
\hline Men / women, $\mathrm{n}$ & $15 / 20$ & $17 / 0$ \\
\hline Age $(\mathrm{y})$ & $40.2 \pm 19.6$ & $59.5 \pm 5.4$ \\
\hline BMI $\left(\mathrm{kg} / \mathrm{m}^{2}\right)$ & $24.7 \pm 2.7$ & $27.2 \pm 1.4$ \\
\hline Weight $(\mathrm{kg})$ & $71.9 \pm 12.1$ & $86.5 \pm 6.5$ \\
\hline Total cholesterol $(\mathrm{mmol} / \mathrm{L})$ & $4.9 \pm 1.1$ & $5.3 \pm 0.7$ \\
\hline Triacylglycerol $(\mathrm{mmol} / \mathrm{L})$ & $1.1 \pm 0.6$ & $1.2 \pm 0.4$ \\
\hline Glucose $(\mathrm{mmol} / \mathrm{L})$ & $5.2 \pm 1.0$ & $5.3 \pm 0.4$ \\
\hline
\end{tabular}

\section{Algae study}

As shown in Table 5.2, IL-6, IL-8 and TNF $\alpha$ concentrations did not differ between the spirulina and placebo conditions $(P=0.671, P=0.421, P=0.122$ respectively $)$, or between the wakame and placebo conditions $(P=0.148,0.484,0.633$ respectively). Moreover, also serum hsCRP concentrations (Table 5.2) did not differ between the spirulina $(P=0.520)$ or wakame $(P=0.116)$ and placebo conditions. When stratifying for serum hsCRP concentrations below $(n=27)$ or above $(n=8)$ $2.0 \mathrm{mg} / \mathrm{L}^{6}$ at the end of the placebo period, still no differences were found between the spirulina and wakame conditions versus placebo. 


\section{CHAPTER 5}

Table 5.2: Mean ( \pm SEM) serum concentrations of interleukin 6 (IL-6), interleukin 8 (IL-8), tumor necrosis factor alpha (TNFa), and high-sensitivity C-reactive protein (hsCRP) after spirulina, wakame and placebo intake $(n=35)$

\begin{tabular}{lccccc}
\hline & Spirulina & Wakame & Placebo & \multicolumn{2}{c}{$\begin{array}{c}\text { Estimated difference } \\
\text { (versus placebo) }\end{array}$} \\
\cline { 5 - 6 } & & & & Spirulina & Wakame \\
\hline $\begin{array}{l}\text { IL-6 } \\
(\mathrm{pg} / \mathrm{mL})\end{array}$ & $0.62 \pm 0.11$ & $0.81 \pm 0.17$ & $0.59 \pm 0.09$ & 0.03 & 0.13 \\
\hline $\mathrm{lL}-8$ & & & & $(-0.14-0.21)$ & $(-0.05-0.30)$ \\
$(\mathrm{pg} / \mathrm{mL})$ & $14.31 \pm 0.83$ & $13.28 \pm 0.85$ & $13.75 \pm 0.89$ & 0.53 & -0.46 \\
\hline $\mathrm{TNFa}$ & & & & $(-0.78-1.85)$ & $(-1.781-0.85)$ \\
$(\mathrm{pg} / \mathrm{mL})$ & $3.55 \pm 0.15$ & $3.41 \pm 0.12$ & $3.40 \pm 0.12$ & 0.19 & 0.06 \\
\hline $\mathrm{hsCRP}$ & $1.95 \pm 0.34$ & $2.36 \pm 0.52$ & $1.69 \pm 0.27$ & $\begin{array}{c}0.27 \\
(-0.05-0.42)\end{array}$ & $(-0.18-0.30)$ \\
$(\mathrm{mg} / \mathrm{L})$ & & & & $0.67-1.11)$ & $(-0.17-1.51)$ \\
\hline
\end{tabular}

${ }^{a}$ Estimated difference and $95 \%$ confidence interval $(\mathrm{Cl})$, based on estimated marginal means obtained with linear mixed models

\section{Goji berry study}

Baseline concentrations of markers of low-grade systemic inflammation did not differ between the two test days (data not shown). Four hours after meal intake, serum IL6 concentrations were significantly increased $(P<0.001$ for factor time, Figure 5.1). However, changes over time were not affected by the Lycium barbarum meal $(\mathrm{P}=$ 0.437 for factor diet; $P=0.372$ for iAUC, Table 5.3).

Table 5.3: Mean ( \pm SEM) iAUCs over 4 hours for serum concentrations of interleukin 6 (IL-6), interleukin 8 (IL-8) and tumor necrosis factor alpha (TNFa) after Lycium barbarum and control meal consumption.

\begin{tabular}{lcc}
\hline & $\begin{array}{c}\text { Lycium barbarum } \\
\text { meal }\end{array}$ & $\begin{array}{c}\text { Control } \\
\text { meal }\end{array}$ \\
\hline $\begin{array}{l}\mathrm{IL}-6 \\
(\mathrm{pg} / \mathrm{mL} \text { per } 240 \mathrm{~min})\end{array}$ & $67.1 \pm 22.3$ & $46.2 \pm 16.2$ \\
\hline $\mathrm{IL}-8$ & $387.9 \pm 156.81$ & $206.6 \pm 44.0$ \\
$(\mathrm{pg} / \mathrm{mL}$ per $240 \mathrm{~min})$ & $26.5 \pm 6.2$ & $24.0 \pm 6.0$ \\
\hline $\begin{array}{l}\mathrm{TNFa} \\
(\mathrm{pg} / \mathrm{mL} \text { per } 240 \mathrm{~min})\end{array}$ & & \\
\hline
\end{tabular}




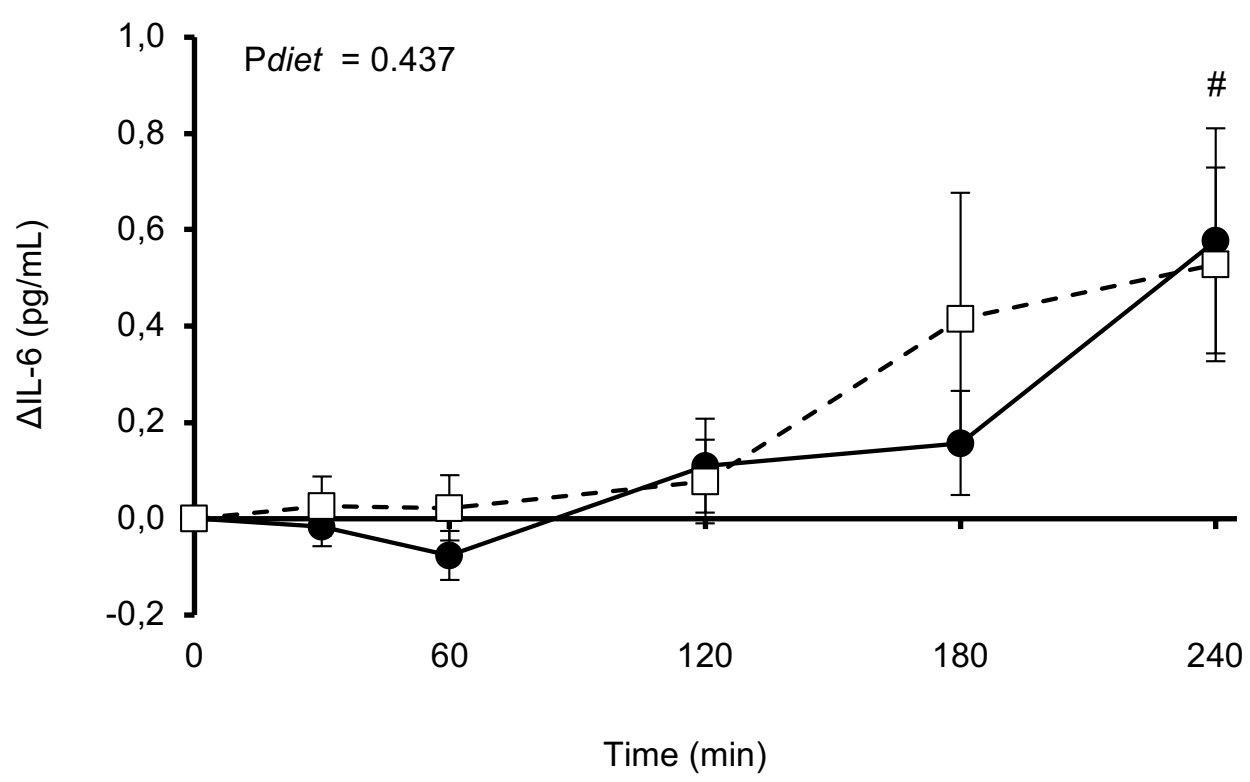

Figure 5.1: Mean changes ( \pm SEM) in interleukin 6 (IL-6) concentrations after Lycium barbarum $(\square)$ and control $(\bullet)$ meal consumption $(n=17)$. Data was analyzed using linear mixed models. After Bonferroni correction, factor time was significantly different from baseline values at 240 minutes $(p<0.001)(\#)$.

Serum IL-8 concentrations were also significantly increased 2 hours after meal intake and remained elevated after 4 hours $(P=0.025$ for factor time, Figure 5.2). In addition, a significant increase in IL-8 concentrations was found after the Lycium barbarum meal $(P=0.003$ for factor diet), although iAUCs did not differ between meals $(P=0.210$, Table 5.3).

Serum TNFa concentrations were not changed after meal intake $(P=0.271$ for factor time, Figure 5.3) and did not differ between meals $(P=0.870$ for factor diet; $P=$ 0.781 for iAUC, Table 5.3). As only one subject had hsCRP concentrations above $2.0 \mathrm{mg} / \mathrm{L}^{6}$ throughout the study, analyses were not repeated after stratification for hsCRP concentrations. 


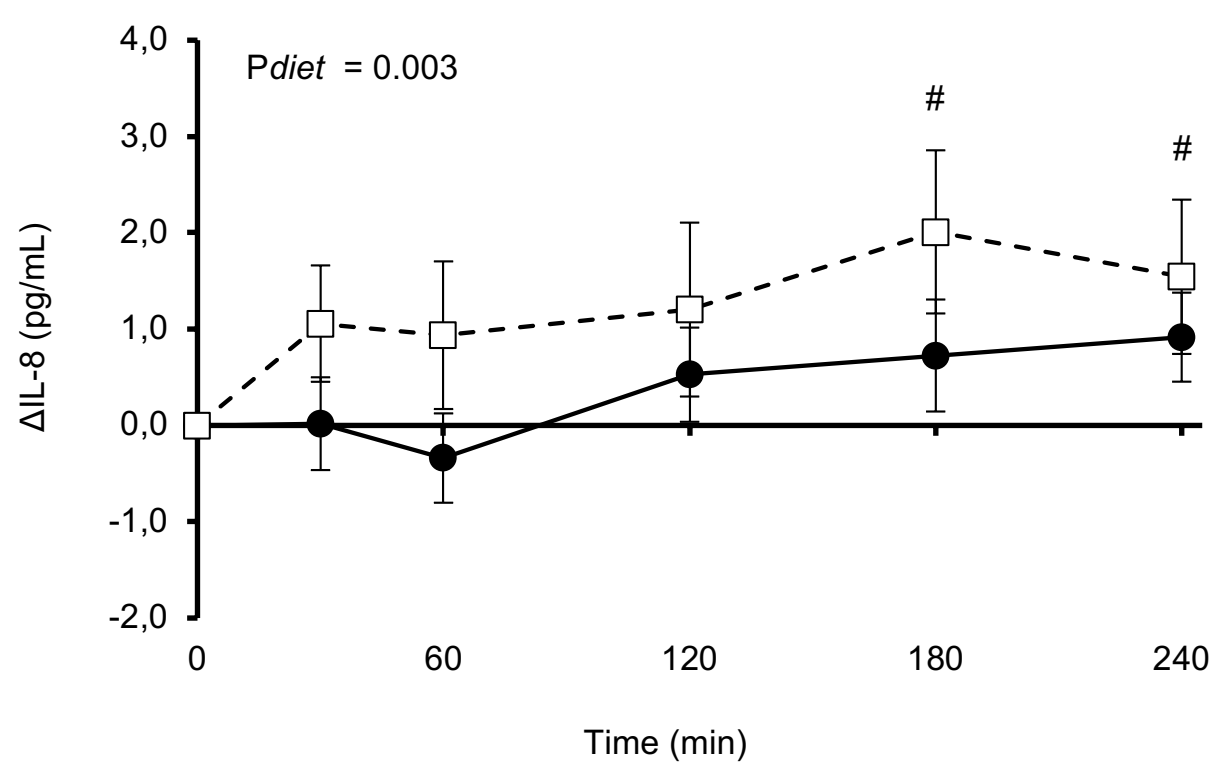

Figure 5.2: Mean changes $( \pm$ SEM) in interleukin 8 (IL-8) concentrations after Lycium barbarum $(\square)$ and control $(\bullet)$ meal consumption $(n=17)$. Data was analyzed using linear mixed models. Significant time effects compared to baseline were found ( $p<0.05$ with Bonferroni correction) (\#).

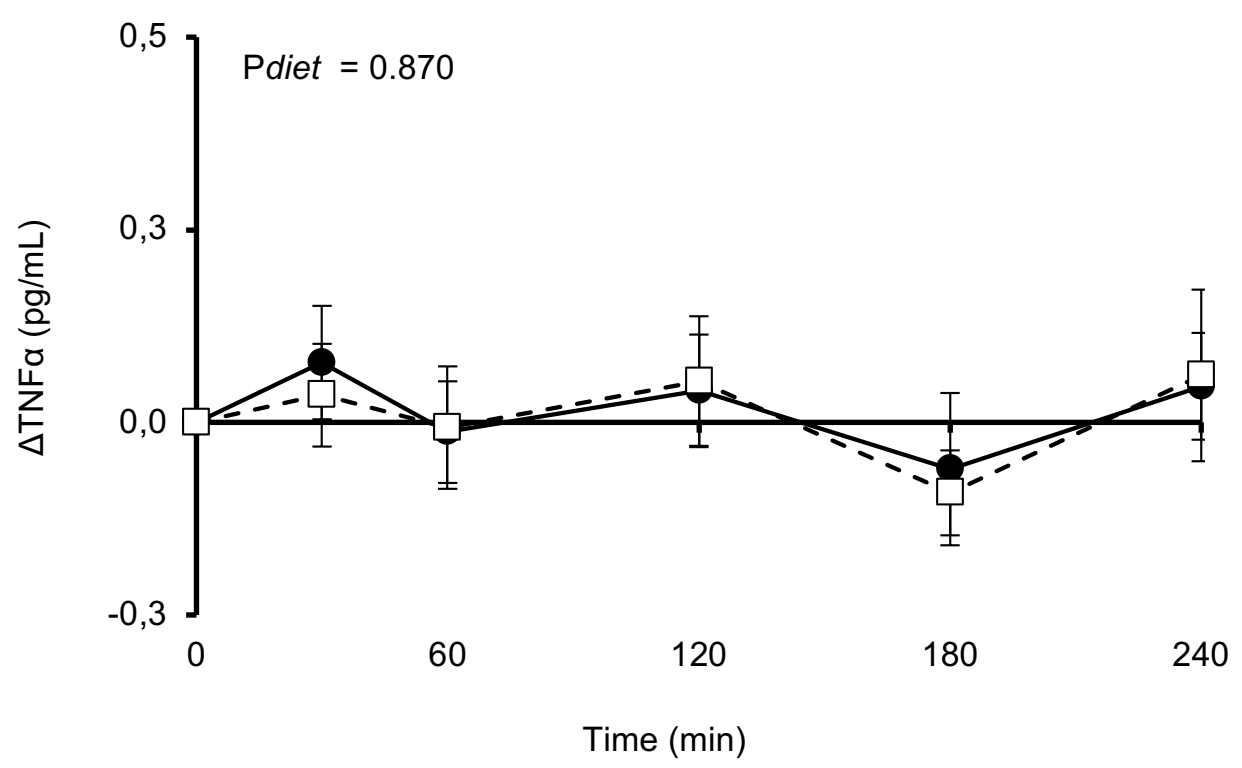

Figure 5.3: Mean changes $( \pm$ SEM) in tumor necrosis factor alpha (TNFa) concentrations after the Lycium barbarum meal $(\square)$ and control meal $(\bullet)$ in 17 healthy overweight men. Data was analyzed using linear mixed models. 


\section{Discussion}

In this study, we found no effects of spirulina or wakame consumption for 17 days on fasting serum IL-6, IL-8, TNFa or hsCRP concentrations, and no effects of a single dose of goji berries on postprandial IL-6 and TNFa concentrations. However, serum IL-8 concentrations increased after intake of a meal with Lycium barbarum as compared to a control meal.

Several in vitro and animal studies have reported anti-inflammatory effects of spirulina consumption. In vitro, pretreatment with a spirulina extract reduced the expression and secretion of TNFa, IL-1 $\beta$ and IL- 6 by LPS-stimulated RAW 264.7 and murine peritoneal macrophages. ${ }^{18,19}$ In animals, expression or secretion of proinflammatory cytokines, including TNF $\alpha$, IL-1 $1 \beta$ and IL-6, was reduced in rats with carrageenan-induced paw oedema, ${ }^{20}$ splenocytes from high-fat diet-fed mice, ${ }^{21}$ and kidney tissue from NIC intoxication treated mice ${ }^{22}$ after spirulina or spirulina extract treatment. However, human studies on the effects of spirulina on circulating inflammatory markers are scarce and not conclusive. In line with our results, serum TNF $\alpha$ and IL-6 concentrations were not altered after spirulina consumption in type II diabetics. ${ }^{2008}$ On the other hand, IL-4 concentrations produced by ex vivo PHAstimulated peripheral blood mononuclear cells (PBMCs) isolated from allergic rhinitis patients were decreased after consuming spirulina for 12 weeks. ${ }^{23}$ In addition, spirulina consumption for 3 months decreased IL-6 concentrations compared to placebo in obese hypertensive subjects, whereas TNFa concentrations were not affected. ${ }^{12}$ In elderly, IL-2 concentrations increased in men and women and IL-6 concentrations decreased in men only after 4 months of spirulina consumption. ${ }^{11}$ No effects on TNFa concentrations were found. Interestingly, the increased IL-2 concentrations upon spirulina consumption ${ }^{11}$ may have an immune stimulatory effect. Indeed, in PBMCs isolated from men above 50 years consuming a spirulina extract formula, production of IL-2 was increased in response to antigen stimulation, whereas IL-4 concentrations decreased. ${ }^{24}$ In addition, concentrated spirulina increased IL-1 $\beta$ and IL-4 production in resting and PHA-stimulated $\mathrm{PBMCs}^{25}$ and a spirulina extract increased mRNA levels of IL-1 $1 \beta$ and TNFa in THP-1 macrophages. ${ }^{26}$ In other words, both anti-inflammatory as well as immune- 
stimulating effects have been reported in stimulated and non-stimulated conditions, which seems contradictive. However, it should be noted that study designs together with outcome parameters differed largely between the human studies and might have contributed to the apparent discrepancies. Effects of spirulina consumption on cytokine production were measured in ex vivo-stimulated conditions ${ }^{24,25}$ and in vivo non-stimulated. ${ }^{11,12,14}$ Outcomes of these two different approaches may not correlate and lead to different conclusions. ${ }^{27}$ In addition, variation in study population adds to the complexity of comparing studies. Spirulina consumption may affect the types of cytokines produced in subjects with a disturbed Th1/Th2 balance, such as allergic rhinitis patients and elderly, ${ }^{28,29}$ thereby explaining the reported effects on IL-4 and IL-2. However, it does not explain the inconsistency in effects on IL-6 concentrations. In populations characterized by low-grade systemic inflammation, ${ }^{30}$ IL-6 concentrations decreased in elderly and obese hypertensives, ${ }^{11,12}$ but not in type II diabetics. ${ }^{14}$ It is, however, an assumption that these populations were characterized by low-grade systemic inflammation. The studies did not report hsCRP concentrations at baseline and this clinical marker would be of interest to compare status of low-grade inflammation between study populations. In our healthy population, no effects were found as well. Intake cannot be the explaining factor, since 8 grams per day yielded both no ${ }^{14}$ as well as IL-6 lowering effects ${ }^{11}$ and 2 grams a day also lowered IL-6 concentrations in vivo. ${ }^{12}$

For wakame, in vitro studies have reported anti-inflammatory effects of the wakame constituents fucoidan and fucosterol. In adipocytes, ${ }^{31}$ LPS-stimulated macrophages ${ }^{32,33}$ and microglial cells, ${ }^{34}$ production of pro-inflammatory cytokines TNF $\alpha$, IL-1 $\beta$ and IL- 6 was inhibited. These results were supported by a study in highfat diet-fed Wistar rats, in which wakame lowered plasma levels of CRP and IL-6 expression in white adipose tissue. ${ }^{9}$ In C57BL/6N mice, however, wakame did not affect plasma IL-6 concentrations. ${ }^{35}$ Only one human trial has evaluated the effects of wakame consumption on markers of low-grade systemic inflammation. In subjects with the metabolic syndrome, consumption of 4 or 6 grams wakame daily for 1 month did not affect CRP concentrations, ${ }^{15}$ which is in line with our study. Although in vitro studies were promising, results cannot be translated to humans. A possible 
explanation for this might relate to dose. Extracts of wakame used in the in vivo experiments were generally less concentrated than those used in in vitro experiments. Also, in vivo metabolism of the extracts may have influenced outcomes.

Acute effects of goji berry consumption on markers of low-grade systemic inflammation have not been investigated in humans yet. However, a few longer-term intervention studies have suggested potential effects. First, in healthy elderly, consumption of a milk-based goji berry formulation for 3 months did not alter circulating hsCRP and IL-6 concentrations. ${ }^{16}$ Second, IL-2 concentrations were increased in Chinese elderly after 30 days of goji berry juice consumption. ${ }^{13}$ Again, this increase in IL-2 concentrations can be considered as an immune-stimulatory effect, which has also been suggested by in vitro studies with Lycium barbarum extracts. PBMCs stimulated with Lycium barbarum polysaccharides (LBPs) increased mRNA levels of IL-2 and TNFa. ${ }^{36}$ In another in vitro experiment, TNFa, IL-2 and IL-4 mRNA expressions, and IL-2 production were increased in LBP-treated mouse splenocytes. However, protein production of TNFa and IL-4 were not affected. ${ }^{37}$ In contrast, consumption of goji berry extracts by overweight subjects decreased blood mRNA levels of TNFa and IL-6. ${ }^{10}$ Although the number of studies is limited, it seems that the Th1/Th2 balance might be affected, whereas results on IL-6 concentrations are contradictory. In addition, the variety in goji berry interventions, e.g. formulation versus extracts, makes it difficult to compare studies.

It has been suggested that increases in pro-inflammatory cytokines after high-fat meal intake relate to elevated CVD risk. ${ }^{38} \mathrm{~A}$ single dose of Lycium barbarum however could not inhibit increases in IL-6 and IL-8 concentrations in the postprandial phase. In contrast, we found an unexpected postprandial increase in IL-8 concentrations compared to control. Since the increase in serum IL-8 concentrations was a single observation and not confirmed by changes in TNFa and IL-6 concentrations, results are difficult to interpret. 
In general, the cytokines measured in the discussed studies vary from T-helper cytokines, including IL-2 (Th1) and IL-4 (Th2), to pro-inflammatory cytokines (TNFa, IL-6, IL-8) and the downstream marker hsCRP. Although T-helper cytokines may play a role in the pathogenesis of atherosclerosis, their link to CVD risk is not well studied. ${ }^{39}$ On the other hand, hsCRP and pro-inflammatory cytokines as markers of low-grade systemic inflammation have been clearly linked to CVD risk. Circulating hsCRP and TNFa concentrations are in fact independent predictors for future vascular events and IL-6 concentrations are correlated with future vascular risk. ${ }^{40}$ Although the association between IL-8 and CVD risk is controversial, ${ }^{41}$ its role in the pathogenesis of atherosclerosis is acknowledged. ${ }^{42}$ For now, we should therefore focus on hsCRP and pro-inflammatory cytokines when assessing CVD risk, until the link between T-helper cytokines, IL-8 and CVD risk is better established.

The study population is important when assessing immunomodulatory activity. Age, for example, might affect immune function and circulating pro-inflammatory cytokine concentrations. ${ }^{43}$ In addition, other populations, such as obese subjects and type II diabetics, are characterized by increased low-grade systemic inflammation. ${ }^{30}$ However, none of the trials discussed, including ours, actually selected their study population based on the presence of low-grade systemic inflammation. An antiinflammatory agent (IL-1 $\beta$ antibody), lowering CRP and IL-6 concentrations also decreased CVD mortality and morbidity in patients with previous myocardial infarction and a residual inflammatory risk. ${ }^{6}$ Only participants with a residual inflammatory risk, defined as CRP concentrations above $2 \mathrm{mg} / \mathrm{L}$, were included. Another anti-inflammatory agent, low-dose methotrexate, was not able to lower CRP and IL-6 concentrations and CVD risk in patients with previous myocardial infarction and additionally type II diabetes or metabolic syndrome. ${ }^{44}$ The study population was not screened for residual inflammatory risk, which resulted in markedly lower median CRP concentrations compared to the other trial. It therefore seems plausible that anti-inflammatory effects of interventions can only be expected in subjects with an inflammatory risk. 
To conclude, 17 days of spirulina or wakame consumption, or a single dose of Lycium barbarum did not lower markers of low-grade systemic inflammation in healthy, non-immunocompromised subjects.

\section{Acknowledgements}

The authors would like to thank Maud Beckers for technical support throughout the study. 


\section{References}

1. Ho KK, Pinsky JL, Kannel WB, Levy D. The epidemiology of heart failure: the Framingham Study. J Am Coll Cardiol. 1993;22(4 Suppl A):6a-13a.

2. Kenchaiah S, Evans JC, Levy D, Wilson PWF, Benjamin EJ, Larson MG, et al. Obesity and the risk of heart failure. N Engl J Med. 2002;347(5):305-13.

3. Ceriello A, Genovese S. Atherogenicity of postprandial hyperglycemia and lipotoxicity. Rev Endocr Metab Disord. 2016;17(1):111-6.

4. Kolovou GD, Watts GF, Mikhailidis DP, Perez-Martinez P, Mora S, Bilianou H, et al. Postprandial hypertriglyceridaemia revisited in the era of non-fasting lipid profiles: executive summary of a 2019 Expert Panel Statement. Curr Vasc Pharmacol. 2019; 17(5):538-540.

5. Kaptoge S, Di Angelantonio E, Pennells L, Wood AM, White IR, Gao P, et al. C-reactive protein, fibrinogen, and cardiovascular disease prediction. N Engl J Med. 2012;367(14):1310-20.

6. Ridker PM, Everett BM, Thuren T, MacFadyen JG, Chang WH, Ballantyne C, et al. Antiinflammatory therapy with canakinumab for atherosclerotic disease. N Engl J Med. 2017;377(12):1119-31.

7. Van den Driessche JJ, Plat J, Konings M, Mensink RP. Effects of spirulina and wakame consumption on intestinal cholesterol absorption and serum lipid concentrations in nonhypercholesterolemic adult men and women. Eur J Nutr. 2019.

8. Van den Driessche JJ, Plat J, Plasqui G, Mensink RP. A single dose of goji berries does not affect postprandial energy expenditure and substrate oxidation in healthy, overweight men. J Nutr Metab. 2019;2019:4057143.

9. Grasa-Lopez A, Miliar-Garcia A, Quevedo-Corona L, Paniagua-Castro N, Escalona-Cardoso G, Reyes-Maldonado $\mathrm{E}$, et al. Undaria pinnatifida and fucoxanthin ameliorate lipogenesis and markers of both inflammation and cardiovascular dysfunction in an animal model of diet-induced obesity. Mar Drugs. 2016;14(8).

10. Lee YJ, Ahn Y, Kwon O, Lee MY, Lee CH, Lee S, et al. Dietary wolfberry extract modifies oxidative stress by controlling the expression of inflammatory mRNAs in overweight and hypercholesterolemic subjects: A Randomized, Double-Blind, Placebo-Controlled Trial. J Agric Food Chem. 2017;65(2):309-16.

11. Park HJ, Lee YJ, Ryu HK, Kim MH, Chung HW, Kim WY. A randomized double-blind, placebocontrolled study to establish the effects of spirulina in elderly Koreans. Ann Nutr Metab. 2008;52(4):322-8.

12. Szulinska M, Gibas-Dorna M, Miller-Kasprzak E, Suliburska J, Miczke A, Walczak-Galezewska M, et al. Spirulina maxima improves insulin sensitivity, lipid profile, and total antioxidant status in obese patients with well-treated hypertension: a randomized double-blind placebo-controlled study. Riv Eur Sci Med Farmacol. 2017;21(10):2473-81.

13. Amagase $\mathrm{H}$, Sun B, Nance DM. Immunomodulatory effects of a standardized Lycium barbarum fruit juice in Chinese older healthy human subjects. J Med Food. 2009;12(5):1159-65.

14. Lee EH, Park JE, Choi YJ, Huh KB, Kim WY. A randomized study to establish the effects of spirulina in type 2 diabetes mellitus patients. Nutr Res Pract. 2008;2(4):295-300. 
15. Teas J, Baldeon ME, Chiriboga DE, Davis JR, Sarries AJ, Braverman LE. Could dietary seaweed reverse the metabolic syndrome? Asia Pac J Clin Nutr. 2009;18(2):145-54.

16. Vidal K, Bucheli P, Gao Q, Moulin J, Shen LS, Wang J, et al. Immunomodulatory effects of dietary supplementation with a milk-based wolfberry formulation in healthy elderly: a randomized, doubleblind, placebo-controlled trial. Rejuvenation Res. 2012;15(1):89-97.

17. Matthews JN, Altman DG, Campbell MJ, Royston P. Analysis of serial measurements in medical research. Br Med J. 1990;300(6719):230-5.

18. Ku CS, Pham TX, Park Y, Kim B, Shin MS, Kang I, et al. Edible blue-green algae reduce the production of pro-inflammatory cytokines by inhibiting NF-kappaB pathway in macrophages and splenocytes. Biochim Biophys Acta. 2013;1830(4):2981-8.

19. Nawrocka D, Kornicka K, Smieszek A, Marycz K. Spirulina platensis improves mitochondrial function impaired by elevated pxidative stress in adipose-derived mesenchymal stromal cells (ASCs) and intestinal epithelial cells (IECs), and enhances insulin sensitivity in equine metabolic syndrome (EMS) horses. Mar Drugs. 2017;15(8).

20. Abu-Taweel GM, Mohsen GA, Antonisamy P, Arokiyaraj S, Kim HJ, Kim SJ, et al. Spirulina consumption effectively reduces anti-inflammatory and pain related infectious diseases. $\mathrm{J}$ Infect Publ Health. 2019.

21. Pham TX, Lee Y, Bae M, Hu S, Kang H, Kim MB, et al. Spirulina supplementation in a mouse model of diet-induced liver fibrosis reduced the pro-inflammatory response of splenocytes. $\mathrm{Br} \mathrm{J}$ Nutr. 2019;121(7):748-55.

22. Zahran WE, Emam MA. Renoprotective effect of Spirulina platensis extract against nicotineinduced oxidative stress-mediated inflammation in rats. Phytomedicine. 2018;49:106-10.

23. Mao TK, Van de Water J, Gershwin ME. Effects of a Spirulina-based dietary supplement on cytokine production from allergic rhinitis patients. J Med Food. 2005;8(1):27-30.

24. Lobner M, Walsted A, Larsen R, Bendtzen K, Nielsen $\mathrm{CH}$. Enhancement of human adaptive immune responses by administration of a high-molecular-weight polysaccharide extract from the cyanobacterium Arthrospira platensis. J Med Food. 2008;11(2):313-22.

25. Mao TK, J VDW, Gershwin ME. Effect of spirulina on the secretion of cytokines from peripheral blood mononuclear cells. J Med Food. 2000;3(3):135-40.

26. Pugh N, Ross SA, EISohly HN, EISohly MA, Pasco DS. Isolation of three high molecular weight polysaccharide preparations with potent immunostimulatory activity from Spirulina platensis, aphanizomenon flos-aquae and Chlorella pyrenoidosa. Planta Med. 2001;67(8):737-42.

27. Maerkedahl RB, Frokiaer H, Stenbaek MG, Nielsen CB, Lind MV, Lundtoft C, et al. In Vivo and ex vivo inflammatory markers of common metabolic phenotypes in humans. Metab Syndr Relat Disord. 2018;16(1):29-39.

28. Sandmand M, Bruunsgaard H, Kemp K, Andersen-Ranberg K, Pedersen AN, Skinhøj P, et al. Is ageing associated with a shift in the balance between Type 1 and Type 2 cytokines in humans? Clin Exp Immunol. 2002;127(1):107-14.

29. Scadding G. Cytokine profiles in allergic rhinitis. Curr Allergy Asthma Rep. 2014;14(5):435. 
30. Donath MY, Shoelson SE. Type 2 diabetes as an inflammatory disease. Nat Rev Immunol. 2011;11(2):98-107.

31. Kim KJ, Lee BY. Fucoidan from the sporophyll of Undaria pinnatifida suppresses adipocyte differentiation by inhibition of inflammation-related cytokines in 3T3-L1 cells. Nutr Res. 2012;32(6):439-47.

32. Kim KJ, Yoon KY, Lee BY. Low molecular weight fucoidan from the sporophyll of Undaria pinnatifida suppresses inflammation by promoting the inhibition of mitogen-activated protein kinases and oxidative stress in RAW264.7 cells. Fitoterapia. 2012;83(8):1628-35.

33. Yoo MS, Shin JS, Choi HE, Cho YW, Bang MH, Baek NI, et al. Fucosterol isolated from Undaria pinnatifida inhibits lipopolysaccharide-induced production of nitric oxide and pro-inflammatory cytokines via the inactivation of nuclear factor-kappaB and p38 mitogen-activated protein kinase in RAW264.7 macrophages. Food Chem. 2012;135(3):967-75.

34. Park HY, Han MH, Park C, Jin CY, Kim GY, Choi IW, et al. Anti-inflammatory effects of fucoidan through inhibition of NF-kappaB, MAPK and Akt activation in lipopolysaccharide-induced BV2 microglia cells. Food Chem Toxicol. 2011;49(8):1745-52.

35. Oh JH, Kim J, Lee Y. Anti-inflammatory and anti-diabetic effects of brown seaweeds in high-fat dietinduced obese mice. Nutr Res Pract. 2016;10(1):42-8.

36. Gan L, Zhang SH, Liu Q, Xu HB. A polysaccharide-protein complex from Lycium barbarum upregulates cytokine expression in human peripheral blood mononuclear cells. Eur J Pharmacol. 2003;471(3):217-22.

37. Chen Z, Kwong Huat Tan B, Chan SH. Activation of T lymphocytes by polysaccharide-protein complex from Lycium barbarum L. Int Immunopharmacol. 2008;8(12):1663-71.

38. Burdge GC, Calder PC. Plasma cytokine response during the postprandial period: a potential causal process in vascular disease? Br J Nutr. 2005;93(1):3-9.

39. Madhumitha H, Mohan V, Deepa M, Babu S, Aravindhan V. Increased Th1 and suppressed Th2 serum cytokine levels in subjects with diabetic coronary artery disease. Cardiovasc Diabetol. 2014;13:1.

40. Ridker PM, Luscher TF. Anti-inflammatory therapies for cardiovascular disease. Eur Heart J. 2014;35(27):1782-91.

41. Velasquez IM, Frumento P, Johansson K, Berglund A, de Faire U, Leander K, et al. Association of interleukin 8 with myocardial infarction: results from the Stockholm Heart Epidemiology Program. Int J Cardiol. 2014;172(1):173-8.

42. Apostolakis S, Vogiatzi K, Amanatidou V, Spandidos DA. Interleukin 8 and cardiovascular disease. Cardiovasc Res. 2009;84(3):353-60.

43. Albers R, Antoine JM, Bourdet-Sicard R, Calder PC, Gleeson M, Lesourd B, et al. Markers to measure immunomodulation in human nutrition intervention studies. Br J Nutr. 2005;94(3):452-81.

44. Ridker PM, Everett BM, Pradhan A, MacFadyen JG, Solomon DH, Zaharris E, et al. Low-dose methotrexate for the prevention of atherosclerotic events. N Engl J Med. 2019;380(8):752-62. 


\section{Supplemental materials}

Supplemental table 5.1: macronutrient composition of the Lycium barbarum and control meal

\begin{tabular}{lcc}
\hline & $\begin{array}{c}\text { Lycium barbarum } \\
\text { meal * }\end{array}$ & $\begin{array}{c}\text { Control } \\
\text { meal }\end{array}$ \\
\hline Energy (kcal) & 684 & 683 \\
\hline Total Fat $(\mathrm{g})$ & 41.8 & 41.9 \\
$($ En\%) & 55 & 55 \\
\hline Carbohydrates $(\mathrm{g})$ & 54.4 & 56.0 \\
(En\%) & 32 & 33 \\
\hline Proteins $(\mathrm{g})$ & 20.3 & 20.3 \\
(En\%) & 12 & 12 \\
\hline
\end{tabular}

Values based on package information.

* Lycium barbarum meal contained $25 \mathrm{~g}$ dried Lycium barbarum 



\title{
CHAPTER 6
}

\begin{abstract}
Effects of oven-dried Rhodospirillum rubrum
intake on serum lipid concentrations and safety parameters in slightly hypercholesterolemic men:

results of a first-in-man randomized, placebocontrolled intervention trial
\end{abstract}

José J. van den Driessche, Ronald P. Mensink, Jogchum Plat 
Embargo as requested

\section{CHAPTER 7}

General discussion 
Embargo as requested 
Embargo as requested

Samenvatting 
Embargo as requested

\section{Valorisation}




\section{Dankwoord}


Het is zover: de kurk mag van de fles, de bitterballen in het frituurvet en de vlaggetjes naar buiten. Het boekje is klaar! Daar wil ik graag een aantal mensen voor bedanken.

Allereerst wil ik mijn promotieteam bedanken. Ronald en Jogchum, dank jullie wel voor alle kansen die jullie mij hebben gegeven, van stage tot promotietraject en de ervaringen en uitdagingen tijdens mijn $\mathrm{PhD}$. Ik heb in die tijd ontzettend veel geleerd, over het vakgebied, maar ook over mezelf. Dank voor jullie vertrouwen, goede begeleiding, alle geworpen blikken en de leuke gesprekken over van alles. Ronald, dankjewel voor je altijd kritische maar rechtvaardige blik en de betrokkenheid. Jogchum, bedankt voor jouw optimisme en enthousiasme, wat mij vaak een extra duwtje in de rug heeft gegeven.

Daarnaast wil ik de beoordelingscommissie bedanken, Prof. dr. L.P.A.J. Schrauwen, Prof. dr. W.H.M. Saris, Prof. dr. R.F. Witkamp, Prof. dr. E.A. Trautwein en dr. S. Baumgartner, voor de tijd en moeite die zij hebben gestoken in het lezen en beoordelen van het proefschrift.

Dan zijn er nog veel mensen die ik wil bedanken en dat doe ik graag aan de hand van een van mijn favoriete vakantiehobby's: een puzzeltje. Dank jullie wel!

\section{Horizontaal}

1. Alle lieve studie, werk en zeilvriendjes en vriendinnetjes die ik heb mogen leren kennen in deze stad. Voor alle afleiding, gezelligheid en goede gesprekken.

3. Lieve schoonfamilie uit de omgeving van deze stad.

5. Buurman-baas, de promotor met de kritische blik. Voor het vertrouwen en de goede begeleiding.

7. Altijd optimistische en enthousiaste promotor. Voor het vertrouwen en de goede begeleiding.

10. Lieve Marijn, mama, voor de onvoorwaardelijke steun en liefde.

11. Zonder deze mensen is ons onderzoek niet mogelijk. 
13. Alle (oud) collega's uit deze groep: Merel, Eva, Elske, Lea, Kylie, Sabine, Nathalie, Maud, Maurice, Peter, Lieve, Ellen, Jordi, Fatma, Maite, Kevin, Matthijs, Tanja, Herman, Sultan, Jehad, Martine, Lotte, Sophie, Charlotte, Cara, Lynn, Bibi, Dorien en Resy. Voor alle fijne samenwerkingen, leuke theepauzes en spectaculaire groepsuitjes.

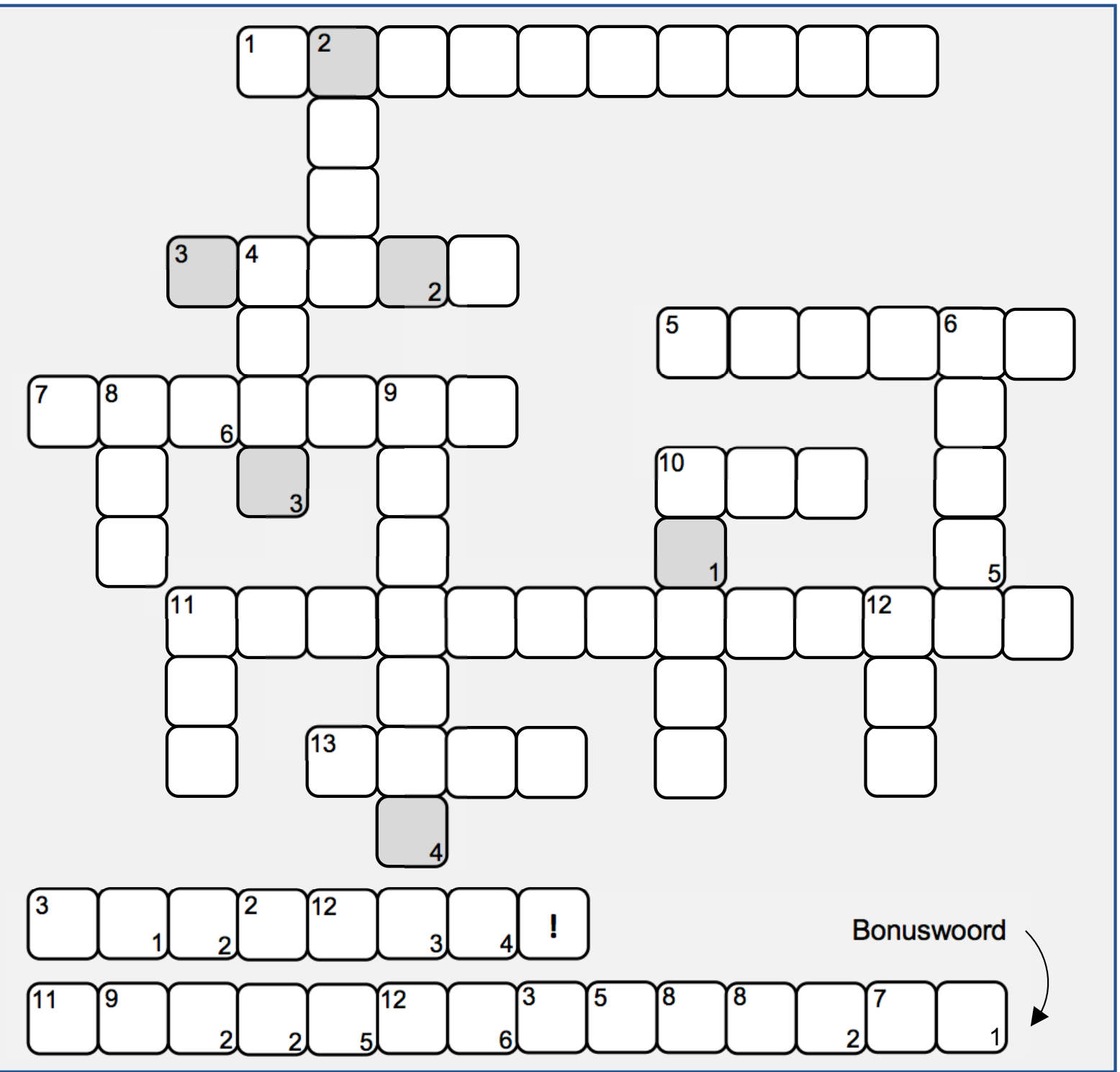




\section{Verticaal}

2. Ook aan de andere kant van de wereld een top vriendinnetje. Voor het paranimf zijn, voor het vriendin zijn.

4. Mijn habibi. Voor alle steun, voor alle liefde.

6. Super zus. Voor de steun en alle geweldige designhulp.

8. $U$ wordt gemist. Voor de eindeloze wijsheden en interesse. Voor de leuke telefoontjes als u de advertenties van collega's in de lokale krant had gevonden.

9. Alle lieve vriendjes en vriendinnetjes vanuit deze stad, mijn hometown. Ook voor alle afleiding, gezelligheid en goede gesprekken.

10. Van kantoorgenootjes, naar enorm goede vriendinnetjes. Voor het paranimf zijn, voor het vriendin zijn.

11. Lieve Henk, papa, voor de onvoorwaardelijke steun en liefde.

12. Collega's van deze afdeling. In het speciaal Guy: voor het beantwoorden van al mijn vragen, de hulp en de leuke gesprekken.

Oplossing op pagina 196. 


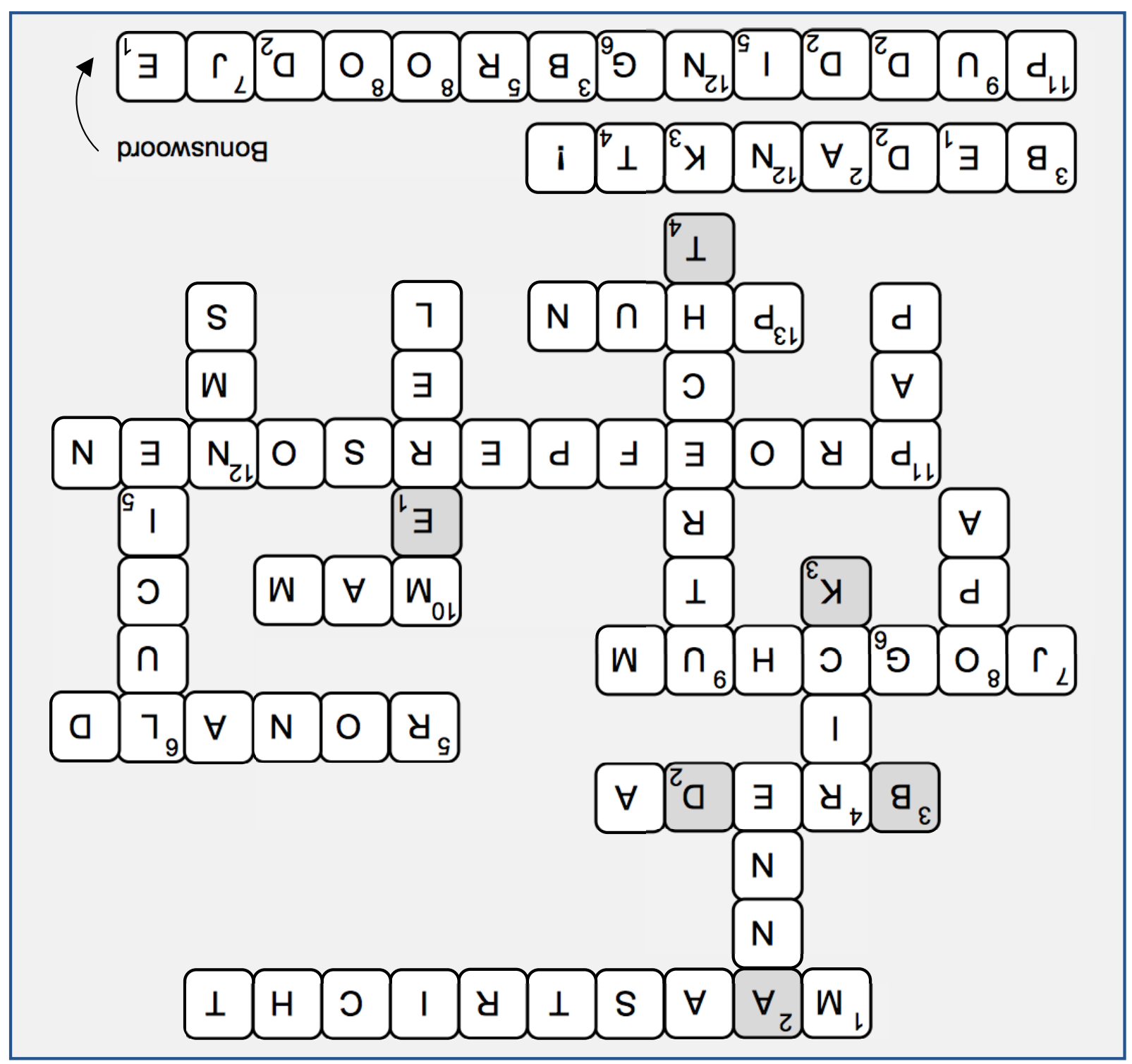


About the author 
José Julia van den Driessche was born on the 27th of July, 1992 in Utrecht, the Netherlands. She completed secondary school at Gerrit Rietveld College in Utrecht in 2010 and moved to Maastricht to study Biomedical Sciences at Maastricht University in the same year. In 2013, she received her bachelor and started her master in Biomedical Sciences at Maastricht University. She performed her junior internship at the department of Human Biology under supervision of prof. dr. Wouter van Marken Lichtenbelt on a project investigating the effects of cold acclimation in type II diabetics. During her senior internship, she worked on a project investigating the effects of theobromine consumption on lipid metabolism and vascular function under the supervision of prof. dr. Jogchum Plat and prof. dr. Ronald Mensink. José started her PhD trajectory in 2015 at the department of Nutrition and Movement Sciences supervised by prof. dr. Ronald Mensink and prof. dr. Jogchum Plat. During her $\mathrm{PhD}$ project, she performed several human intervention trials investigating the effects of goji berries, algae and Rhodospirillum rubrum on cardiometabolic health. She joined the Obesity and Diabetes expert group on the "Establishment of the Efficacy of Intervention in those with the Metabolic Syndrome" from the International Life Sciences Institute Europe in 2017. In 2018, she also joined the Young committee of the Dutch Academy for Nutritional Sciences. 


\section{List of publications}




\section{Accepted manuscripts}

Van den Driessche JJ, Plat J, Konings MCJM, Mensink RP. Effects of spirulina and wakame consumption on intestinal cholesterol absorption and serum lipid concentrations in non-hypercholesterolemic adult men and women. Eur J Nutr. 2019. doi: 10.1007/s00394-019-02073-7.

Van den Driessche JJ, Plat J, Plasqui G, Mensink RP. A single dose of goji berries does not affect postprandial energy expenditure and substrate oxidation in healthy, overweight men. J Nutr Metab. 2019;2019:4057143.

Smolders L, Mensink RP, van den Driessche JJ, Joris PJ, Plat J. Theobromine consumption does not improve fasting and postprandial vascular function in overweight and obese subjects. Eur J Nutr. 2019;58(3);981-7.

Van den Driessche JJ, Plat J, Mensink RP. Effects of superfoods on risk factors of the metabolic syndrome: a systematic review of human intervention trials. Food Funct. 2018;9(4):1944-1966.

Hanssen MJ, Hoeks J, Brans B, van der Lans AA, Schaart G, van den Driessche JJ, Jorgensen JA, Boekschoten MV, Hesselink MK, Havekes B, Kersten S, Mottaghy FM, van Marken Lichtenbelt WD, Schrauwen P. Short-term cold acclimation improves insulin sensitivity in patients with type 2 diabetes mellitus. Nature Med. 2015;21(8).

\section{To be submitted}

Van den Driessche JJ, Mensink RP, Plat J. Spirulina, wakame or goji berries do not lower markers of low-grade systemic inflammation in healthy subjects.

Van den Driessche JJ, Mensink RP, Plat J. Effects of oven-dried Rhodospirillum rubrum intake on serum lipid concentrations and safety parameters in slightly hypercholesterolemic men: results of a first-in-man randomized, placebo-controlled intervention trial. 